\title{
29. CENOZOIC PLANKTONIC FORAMINIFERAL BIOSTRATIGRAPHY OF THE SOUTHWESTERN ATLANTIC (RIO GRANDE RISE): DEEP SEA DRILLING PROJECT LEG 721
}

\author{
C. Pujol, Département de Géologie et Océanographie, Université de Bordeaux I, 33405 Talence, France
}

\begin{abstract}
Our study focuses on the biostratigraphy of Cenozoic planktonic foraminifers sampled during Leg 72 . We located the sites of interest on the Rio Grande Rise and its surroundings (southwestern Atlantic). A rich assemblage of the microfauna and the recovery of a good Cenozoic sequence of fered an opportunity for gathering documentary evidence on the planktonic biostratigraphy of this area.

Site 515 was drilled in the Brazil Basin at a depth of $4250 \mathrm{~m}$. The planktonic foraminifers were generally poorly preserved, with the exception of a short lower Eocene sequence at the base of Hole 515B. The equivalent of Zones P9.to P6b were identified at the base of Hole 515B.

Holes 516 and 516A and Sites 517 and 518 contain a good Pliocene sequence in which Subzones PL1 to PL6 were differentiated. At Hole 516, the cores range from lower to upper Miocene, and there is probably a brief gap at the base of the upper Miocene. Despite the extensive lower Miocene section, stratigraphic refinement according to Blow's zones was difficult because of a high-latitude component. The spiny morphotype of Globoquadrina as in Site 357 (PerchNielsen et al., 1977) and Globorotalia zealandica group are the typical components of this lower Miocene high-latitude fauna. G. miozea conomiozea morphotypes have a long stratigraphic range occurring from lower-middle Miocene to the base of the Pliocene.

Hole $516 \mathrm{~F}$ contains a complete succession between the basal Miocene and the Cretaceous/Tertiary boundary. Again, stratigraphic definition for the Eocene-Oligocene is somewhat difficult because of the high-latitude fauna. The zones between P10 and P20 are difficult to differentiate on species data. Orbulinoides beckmanni is not recognized, and some genera like Hantkenina seem to have an unusual stratigraphic distribution. In the middle and upper Eocene, Globigerinita forms are very common. The Globigerinatheka genus is also well represented, especially by $G$. subconglobata and $G$. index. Such an assemblage is typical for a temperate-water fauna. A thick Cretaceous slump occurs in the lower Eocene and disturbs the microfauna near the Paleocene/Eocene boundary. Finally, the Paleocene section is complete, allowing differentiation of Zone P1 in spite of bioturbation and reworked sediment.
\end{abstract}

\section{INTRODUCTION}

DSDP Leg 72 investigated the western part of the Atlantic Ocean near and on the Rio Grande Rise. This leg adds to the information and results already obtained during Leg 3 (Maxwell, Von Herzen, et al., 1970) and Leg 39 (Supko, Perch-Nielsen, et al., 1977).

During Leg 72, four sites were drilled (Fig. 1). The principal objectives of this cruise were to investigate the paleocirculation of the South Atlantic and the tectonic history and evolution of the Rio Grande Rise. Within this general frame of reference, the different sites and holes were arranged in a depth sequence along the eastern flank of the Vema Channel (Sites 517 and 518), in the Brazil Basin (Site 515), and on the Rio Grande Rise (Site 516).

The different holes form a biosedimentary sequence from the Cretaceous period to the present day. The recovery of a practically complete Cenozoic sequence in this area and the importance of the planktonic microfauna offer an opportunity for gathering information on the planktonic biostratigraphy of the southwestern Atlantic.

This contribution describes the Cenozoic planktonic foraminiferal fauna of Sites 515 to 518 . Its aim is bio-

\footnotetext{
${ }^{1}$ Barker, P. F., Carlson, R. L., Johnson, D. A., et al., Init. Repts. DSDP, 72: Washing ton (U.S. Govt. Printing Office)
}

stratigraphic, and it encompasses the entire Cenozoic succession of these four sites, leading to the description of the different biozones.

\section{MATERIAL AND METHODS}

Three of the four Sites drilled during Leg 72 provided well or relatively well preserved fauna. The other site (515), situated in a deep water area, was often barren because of the dissolution effects in most of the sequence. Figure 1 shows the locations of the sites and a list is given below.

\begin{tabular}{lccc}
\hline Hole & Latitude (S) & Longitude (W) & $\begin{array}{c}\text { Water } \\
\text { depth (m) }\end{array}$ \\
\hline 515 & $26^{\circ} 14.33^{\prime}$ & $36^{\circ} 30.17^{\prime}$ & 4250.4 \\
$515 \mathrm{~A}$ & $26^{\circ} 14.31^{\prime}$ & $36^{\circ} 30.17^{\prime}$ & 4252.01 \\
$515 \mathrm{~B}$ & $26^{\circ} 14.32^{\prime}$ & $36^{\circ} 30.19^{\prime}$ & 4252.0 \\
516 & $30^{\circ} 16.58^{\prime}$ & $35^{\circ} 17.11^{\prime}$ & 1313.0 \\
$516 \mathrm{~A}$ & $30^{\circ} 16.59^{\prime}$ & $35^{\circ} 17.12^{\prime}$ & 1313.0 \\
$516 \mathrm{~F}$ & $30^{\circ} 16.59^{\prime}$ & $35^{\circ} 17.10^{\prime}$ & 1313.0 \\
517 & $30^{\circ} 56.81^{\prime}$ & $38^{\circ} 02.47^{\prime}$ & 2963.0 \\
518 & $2958.42^{\prime}$ & $38^{\circ} 08.12^{\prime}$ & 3944.0 \\
\hline
\end{tabular}

This study is based on core catcher samples or on one sample per core section and on samples taken at closer intervals near biostratigraphic boundaries. Sample volume is about $10 \mathrm{~cm}^{3}$. All the samples were soaked and, if necessary, treated with hydrogen peroxide; or boiled in a solution of washing soda. Then the material was washed over a screen having an average mesh of $0.150 \mathrm{~mm}$ (sometimes a mesh of $0.063 \mathrm{~mm}$ ). The washed residue was then dried, and remarks on planktonic foraminifers were made for each sample, most often on the coarse fraction and more rarely on fine fraction. 
C. PUJOL

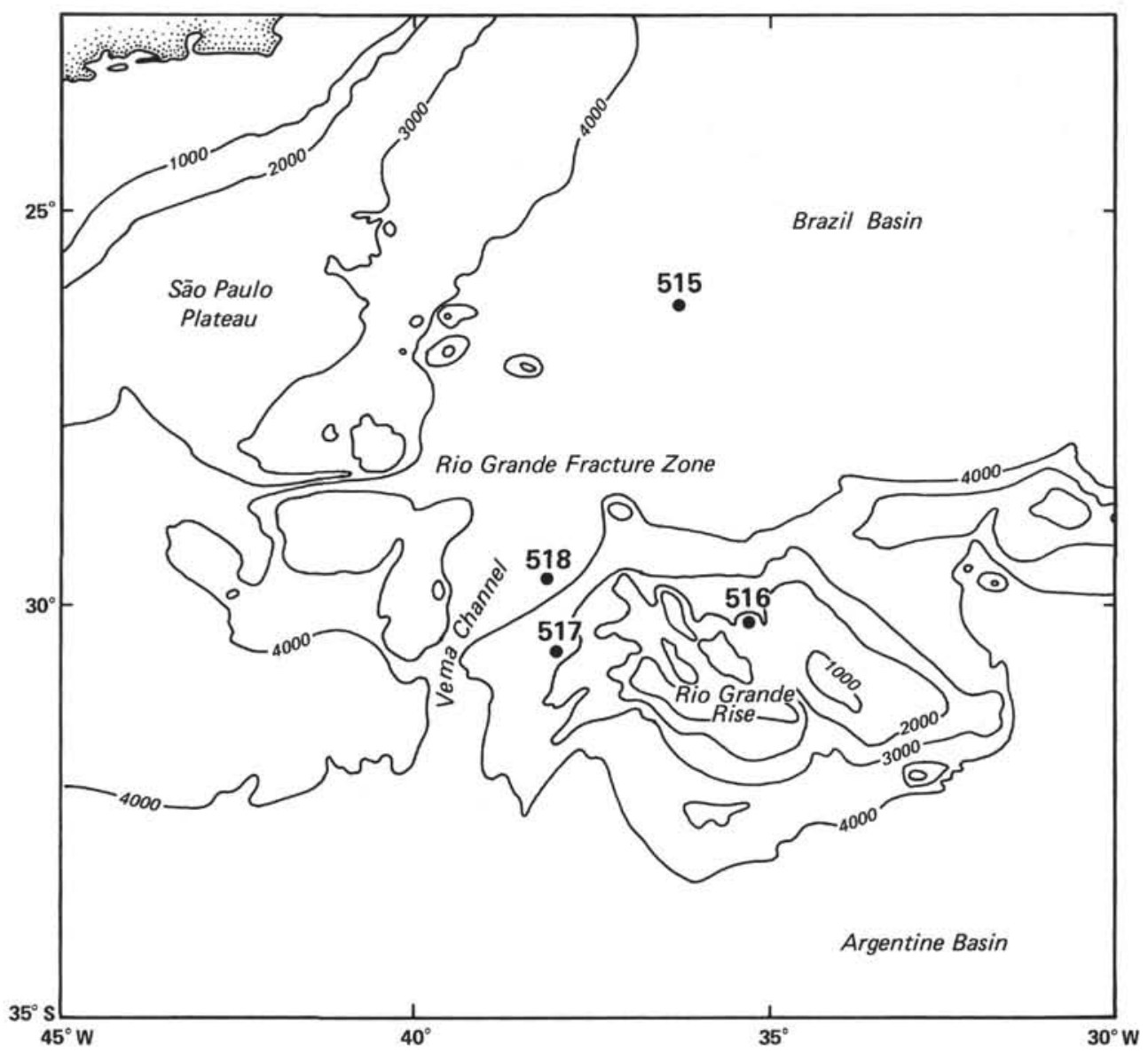

Figure 1. Location of Leg 72 sites.

\section{SITE 515}

Three holes were drilled at this site located in the Brazil Basin in a water depth of $4250 \mathrm{~m}$. The combined cores contain a stratigraphic sequence ranging from the Quaternary (Holes 515 and 515A) through the lower Eocene (Hole 515B).

\section{Hole 515}

Two cores were drilled. They consist of a grayish brown terrigenous mud. The oldest microfossil-bearing sediment cored is at $49 \mathrm{~m}$ sub-bottom, and the assemblages are strongly dissolved. On the basis of Globorotalia truncatulinoides and G. menardii s.l. (left coiling), the assemblage is late Pleistocene (Table 1). Subtropical and tropical species dominate the planktonic assemblage, but the presence of sub-Antarctic species (Globigerina pachyderma) suggests water mass mixing during the late Pleistocene.

\section{Hole 515A}

Twenty-seven cores were recovered from this hole. The sediment lithology of all these cores corresponds to Unit 1 (see site chapter, Site 515, this volume). Planktonic foraminifers are present only in Cores 1 to 10 (Table 2) and generally are poorly preserved. The oldest sediment with planktonic foraminifers is at a sub-bot- tom depth of $42 \mathrm{~m}$. A list of the barren samples is given in the appendix at the end of the chapter.

Cores 1 to 9 contain a typical Quaternary assemblage with Globorotalia truncatulinoides. The presence of rare Globorotalia tosaensis in Section 515A-8-3, preceding Globorotalia triangula $(515 \mathrm{~A}-9, \mathrm{CC})$ places this section in the lower Pleistocene or probably in the Globorotalia crassula Subzone (Pujol and Duprat, this volume).

\section{Hole 515B}

Fifty-seven cores were recovered from Hole 515B, and their lithology is defined in the site chapter for Site 515 (this volume). From top to bottom they are: Unit 1 (Cores 1 to 9), greyish brown terrigenous mud with occasional nannofossil-rich layers and some foraminiferrich layers; Unit 2 (Cores 10 to 55), dark greenish grey siliceous mud grading down to a siliceous (Cores 10 to 46) and terrigenous mudstone (Cores 47 to 55); Unit 3 (Cores 56 and 57), greenish grey calcareous zeolitic mudstone.

Assemblages of planktonic foraminifers are very poor or absent (see Appendix at the end of the chapter), except in the lower cores from this hole. The distribution of these foraminifers is given in Table 3. In the upper part of the hole, only Cores 4 and 6 contain rare specimens of Globigerina pachyderma, Globoquadrina dehiscens, and Globorotalia inflata/puncticulata. This association indicates probably a Pliocene-Quaternary age. No planktonic foraminifers were recovered be- 
Table 1. Distribution of planktonic foraminifers at Hole 515.

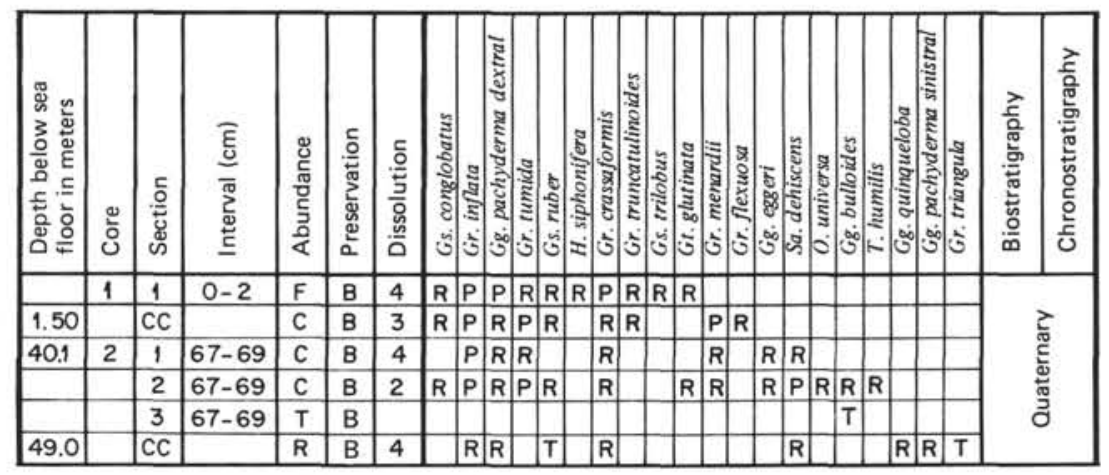

Table 2. Distribution of planktonic foraminifers at Hole 515A.

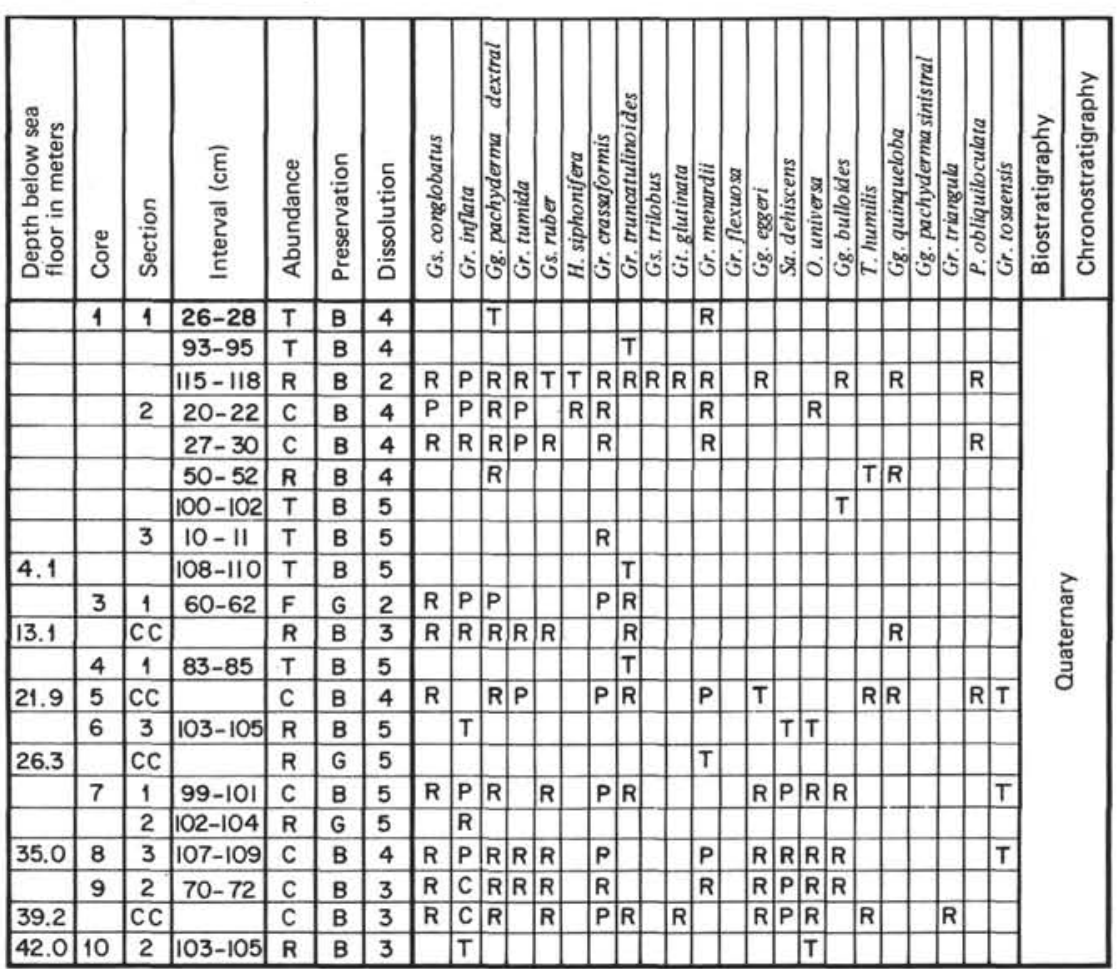

Note: Because of the complexity of Tables 1-8, we have used an unusual format. Below is a list of genus abbreviations and key symbols for these tables.

$\begin{array}{llllll}\text { C } & \text { Catapsydrax } & \text { Gs } & \text { Globigerinoides } & \text { Ps } & \text { Pseudohastigerina } \\ \text { Cd } & \text { Candeina } & \text { Gt } & \text { Globigerinita } & \text { Pu } & \text { Pulleniatina } \\ \text { Gd } & \text { Globorotaloides } & \text { Gtheka } & \text { Globigerinatheka } & \text { Sa } & \text { Sphaeroidinella } \\ \text { Gg } & \text { Globigerina } & \text { H } & \text { Hastigerina } & \text { Ss } & \text { Sphaeroidinellopsis } \\ \text { Gq } & \text { Globoquadrina } & \text { O } & \text { Orbulina } & \text { T } & \text { Turborotalita } \\ \text { Gr } & \text { Globorotalia } & \text { Pr } & \text { Praeorbulina } & \text { To } & \text { Truncorotaloides }\end{array}$

Species abundance is classified as follows: $A=$ abundant, $C=$ common, $F=$ few, $R=$ rare, $\mathrm{T}=$ trace, and blank $=$ barren. Preservation is identified as $\mathrm{G}=\operatorname{good}, \mathrm{M}=$ moderate, and $\mathbf{B}=$ bad; these categories mean that this microfaunal aspect renders specific determination difficult, very difficult, or almost impossible, respectively.

The seventh column from the left specifies the dissolution of tests: blank $=$ not affected, $1=$ slightly affected, $2=$ damaged, $3=$ strongly damaged, $4=$ partly destroyed, $5=$ destroyed.

Planktonic foraminifers are plotted from the base of the hole, in order of appearance. Frequency symbols represent occurrence as follows: A (abundant) $=$ over $30 \% ; \mathrm{C}$ (common) $=$ $15-30 \%$; P (present) $=3-15 \%$; R (rare) $=$ less than 3\%; T (trace) $=$ isolated specimens.

Stratigraphy is indicated on the right-hand column in each table. The zonation used for stratigraphic age determination of the planktonic foraminifers is Blow's (1969) for the upper Tertiary and Berggren's (1971) for the lower Tertiary. When different index taxa are not recognized and subzonation is more detailed, we used other zonation systems; Berggren $(1973,1977 \mathrm{a}, \mathrm{b})$ for the Pliocene; Bolli (1966) and Stainforth and others (1975) for the upper Eocene and the Oligocene. Parentheses around zone numbers indicate that the definitions of the zones are not based on the original data. 


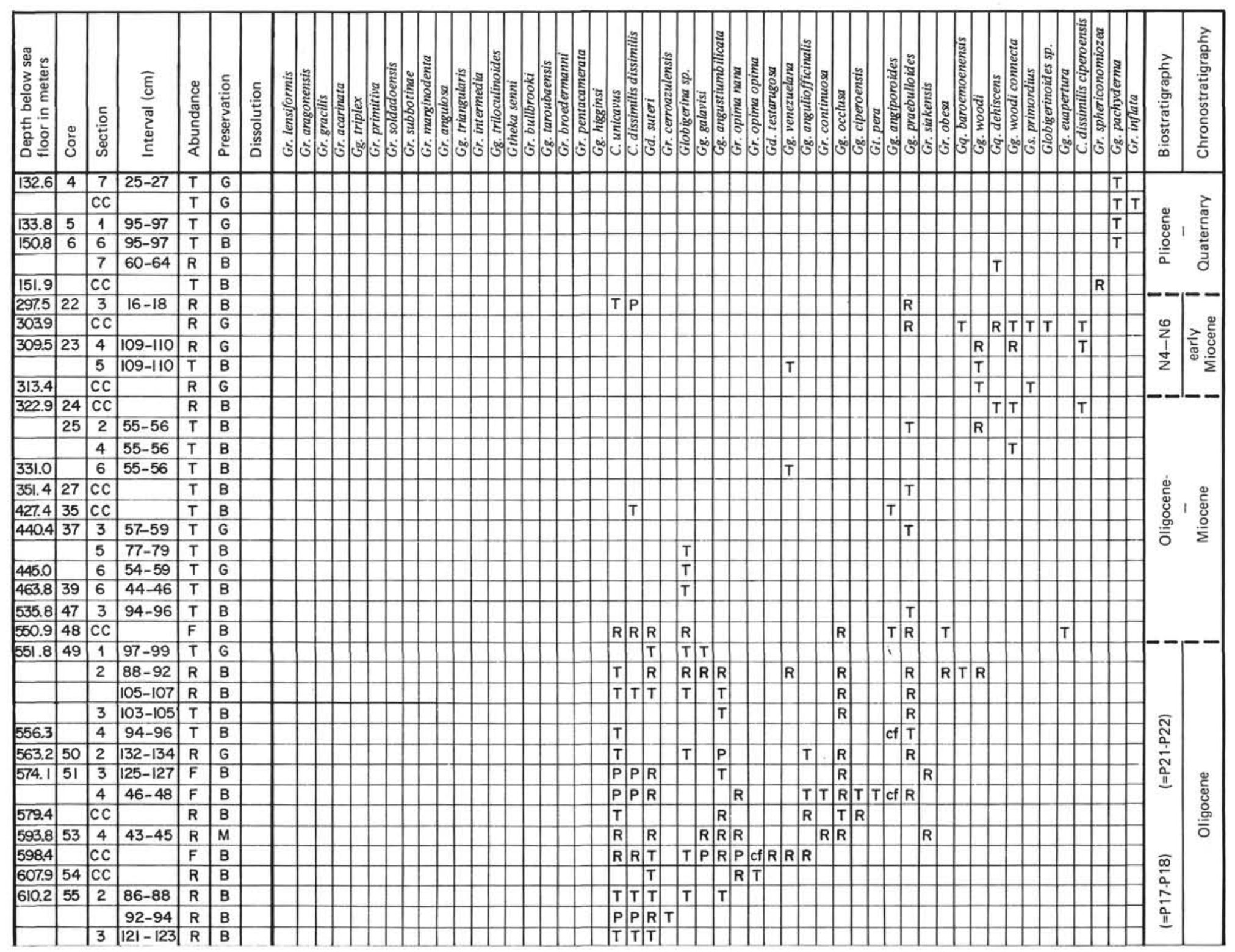


Table 3. (Continued).

\begin{tabular}{|c|c|c|c|c|c|c|c|c|c|c|c|c|c|c|c|c|c|c|c|c|c|c|c|c|c|c|c|c|c|c|c|c|c|c|c|c|c|c|c|c|}
\hline 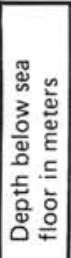 & ๖ั & 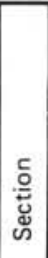 & 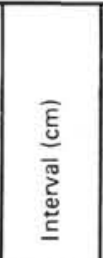 & 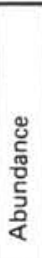 & 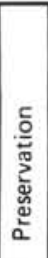 & 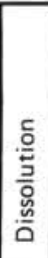 & : & 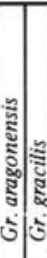 & $\begin{array}{ll} & \\
\vdots \\
\vdots\end{array}$ & 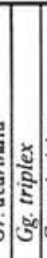 & 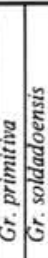 & 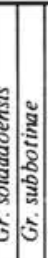 & 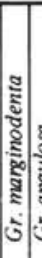 & & 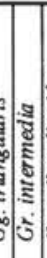 & 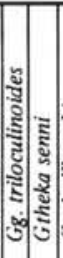 & 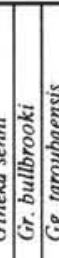 & 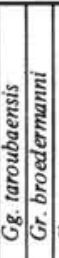 & 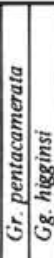 & : & 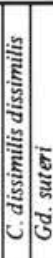 & : & 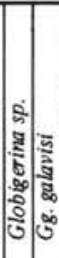 & 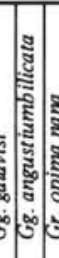 & 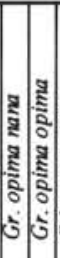 & 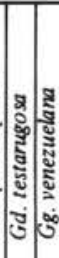 & : & 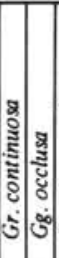 & : & งे & 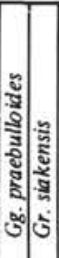 & 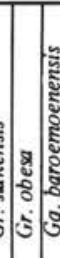 & 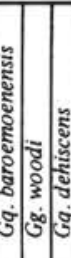 & 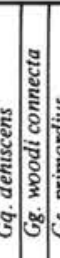 & 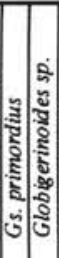 & : & 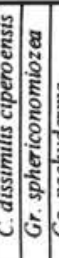 & 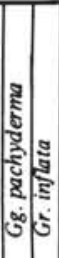 & 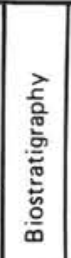 & 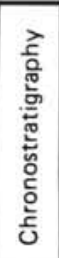 \\
\hline 612.3 & 55 & 3 & $148-150$ & $F$ & $B$ & & & & & & & & & & & & & & & $P$ & $P R$ & & & & & & & & & & & & & & & & & & \multicolumn{2}{|c|}{ Oligocene } \\
\hline 622.7 & 56 & 4 & $80-85$ & C & $B$ & 2 & & $\mathrm{P}$ & A & at & & 8 & & & & & $\mathrm{P} / \mathrm{F}$ & $R$ & & \begin{tabular}{l|l}
$R$ & \\
\end{tabular} & & & & & & & & & & & & & & & & & & & $-\bar{\omega}$ & \multirow{9}{*}{ 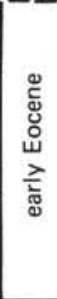 } \\
\hline \multirow[t]{3}{*}{623.6} & & 5 & $21-27$ & C & $B$ & 2 & & $R$ & C & & $R$ & 8 & & $R$ & & $R$ & \begin{tabular}{l|l} 
& \\
\end{tabular} & \begin{tabular}{|l|l|}
$R$ & \\
\end{tabular} & & R & & & & & & & & & & & & & & & & & & & 章 & \\
\hline & & & $142-147$ & C & B & 3 & & $\mathbf{R}$ & $R$ & 8 & & $\mathrm{R}$ & & $R$ & & & $R|c| R$ & \begin{tabular}{l|l|}
$R$ & $T$ \\
\end{tabular} & $T$ & & & & & & & & & & & & & & & & & & & & & \\
\hline & & 6 & $47-52$ & $F$ & $M$ & 2 & & \begin{tabular}{l|l}
$T$ & $R$ \\
\end{tabular} & \begin{tabular}{l|l}
$R$ & $P$ \\
\end{tabular} & & \begin{tabular}{l|l}
$R$ & $R$ \\
\end{tabular} & R & & $\mathrm{R}$ & & & cf & & & & & & & & & & & & & & & & & & & & & & 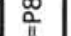 & \\
\hline \multirow{4}{*}{626.9} & & CC & & C & $B$ & 2 & & & A & A. & $\mathbf{R}$ & & & & & $\mathrm{R}$ & $8 \mid$ & & & & & & & & & & & & & & & & & & & & & & & \\
\hline & 57 & 2 & $78-85$ & $\mathrm{~F}$ & B & 3 & $\mathbf{R}$ & $\begin{array}{lll}T & R \\
\end{array}$ & $\begin{array}{l}\mathrm{R} \\
\mathrm{R}\end{array}$ & $R$ & \begin{tabular}{l|l}
$R$ & $R$ \\
\end{tabular} & $\begin{array}{ll}R \\
R\end{array}$ & $R$ & & & & & & & & & & & & & & & & & & & & & & & & & & P7] & \\
\hline & & 4 & $92-98$ & $F$ & B & 2 & $\mathbf{R}$ & & A & $A$ & \begin{tabular}{l|l}
$R$ & $R$ \\
\end{tabular} & $\begin{array}{ll}8 \\
\end{array}$ & & & & & & & & & & & & & & & & & & & & & & & & & & & \multirow{3}{*}{ P6b } & \\
\hline & & 6 & $32-37$ & $\mathrm{~F}$ & $B$ & 1 & $R$ & & & & \begin{tabular}{l|l}
$\mathrm{P}$ & $\mathbf{2}$
\end{tabular} & $\mathrm{P}$ & & \begin{tabular}{l|l}
$R$ & $R$ \\
\end{tabular} & $|R|$ & & & & & & & & & & & & & & & & & & & & & & & & & \\
\hline 636.4 & & $\mathrm{CC}$ & & C & $B$ & 1 & c & & $\begin{array}{ll}\mathrm{R} & \mathrm{C} \\
\end{array}$ & $R$ & \begin{tabular}{l|l}
$P$ & $R$ \\
\end{tabular} & P & \begin{tabular}{|l|l}
$R$ & $F$ \\
\end{tabular} & $\begin{array}{l}R \\
R\end{array}$ & 1 & $P$ & & & & & & & & & & & & & & & & & & & & & & & & \\
\hline
\end{tabular}

Note: Please see note on Table 2. 
tween Cores 7 and 21. In Cores 22 and 23, the assemblages observed contain Catapsydrax dissimilis ciperoensis, Globoquadrina dehiscens, Globigerinoides primordius, indicative of lower Miocene Zones N4 to N6. Between Cores 24 to 48 , the few species present have long ranges indicating an Oligocene-Miocene age. The next cores (49 to 55) contain an Oligocene assemblage without $G$. primordius and with few Globigerina anguliofficinalis and Globorotalia opima opima, approximately between $550 \mathrm{~m}$ and $612 \mathrm{~m}$ sub-bottom depth. A single specimen of Globorotalia cerroazulensis in Sample 515B-55-2, 92-94 cm places this sample in Zones P17 to P18. The different taxa observed in Cores 51 to 49 indicate an age range spanning Zones P21 to N3. Finally, Cores 56 and 57 show a large lower Eocene assemblage with improved preservation. The succession of the microfauna in the different samples of these two cores makes possible the differentiation of Zones P9 to P6b.

The equivalent to Zone P9 occurs in Sample 515B-565, 21-27 $\mathrm{cm}$ based on the presence of Globigerina higginsi associated with the other species below. Zone P8 is probably found in Sections 5 (base) and 6 of Core 56, and is differentiated by the occurrence of Globigerina angulosa, Globorotalia broedermanni, and other species. The appearance of Globorotalia aragonensis in Section 515B-57-2 suggests Zone P7. The association of Globorotalia subbotinae and Globorotalia lensiformis without $G$. aragonensis in Core 57, Sections 4 to 6 indicates Zone P6b.

\section{SITE 516}

Three successful holes were drilled at this site, located on the Rio Grande Rise at a water depth of $1313 \mathrm{~m}$. The cores recovered contain a stratigraphic sequence extending from the Quaternary (Holes 516 and 516A) to the Upper Cretaceous (Santonian/Coniacian) (Hole 516F), terminating in basalt at a sub-bottom depth of $1270.6 \mathrm{~m}$.

\section{Hole 516}

Forty-four cores were recovered from this hole. The oldest sediments are lower Miocene from a sub-bottom depth of $183.3 \mathrm{~m}$. The sediments from Hole 516 are loosely consolidated nannofossil ooze. The planktonic foraminifers are abundant and well preserved. Their distribution is given in Table 4.

Core 516-1 to Section 516-2-2 contain a typical Quaternary association. In the sedimentary sequence, it was possible to distinguish several biozones defined by the frequencies of Globorotalia crassaformis and Globigerina eggeri and by the distribution of Globorotalia hirsuta and Globorotalia crassula (Pujol and Duprat, this volume).

A Pliocene association occurs between Section 516-3-3 and 516-12,CC. The Pliocene/Quaternary boundary coincides with the disappearance of Globorotalia exilis/ pertenuis and the occurrence of Globorotalia truncatulinoides. In this Pliocene sequence, we determined the different zones already defined by Berggren (1973, $1977 \mathrm{a}, \mathrm{b})$. The base of Zone PL1b, characterized by the occurrence of Globorotalia puncticulata, is in Sample $516-10-3,60-62 \mathrm{~cm}$. Below this point, it has not been possible to differentiate Zone PLla from the $G$. dehiscens/G. margaritae Zone because of the nonsimultaneous distribution in our samples of Globoquadrina dehiscens and Globorotalia margaritae. The base of the Pliocene is therefore established according to Berggren's (1973) model, at the last appearance of Globoquadrina dehiscens (Sample 516-13-1, 90-92 cm).

Cores 13 and 14 are attributed to the upper Miocene. G. dehiscens, Globorotalia conoidea, G. conomiozea, G. lenguaensis, and Sphaeroidinellopsis seminulina characterize this sequence. The disappearance of Globorotalia siakensis determines the top of Zone N14 in Section 516-15-1, suggesting that Cores 13 and 14 could be attributed to Zones N15 to 17. The first appearance of Globigerina nepenthes at Sample 516-16-1, 140-142 cm marks the base of Zone N14. Between this datum and the first occurrence of Orbulina universa in Sample 516-17-3, $50-52 \mathrm{~cm}$ (the base of N9), no differentiation is possible.

Below, an extensive lower Miocene sequence is observed. Globigerinoides sicanus becomes common, and Praeorbulina transitoria first appears in Core 516-22, continuing uphole to Cores 21 and 20. Moreover, Catapsydrax dissimilis ciperoensis last occurrence in Core 27 and a few isolated specimens in Cores 516-26 and 516$25, \mathrm{CC}$ mark a reduced $\mathrm{N} 7 \mathrm{Z}$ Zone. Taking into account the distribution of high-latitude species like Globorotalia zealandica, we put the top of the equivalent to Zone N7 at the last appearance datum (LAD) of $G$. zealandica (Sample 516-24-2, 70-72 cm). The top of Zone N6 is retained in Core 27. In the absence of Globigerinatella insueta, no differentiation can be made between Zones N5 and N6. Finally, the disappearance of Globorotalia kugleri marks the base of Zone N5 in Core 40.

Drilling ended in Zone $\mathrm{N} 4$ at $183.4 \mathrm{~m}$ sub-bottom. Thus, the lower Miocene is complete and well developed. This extensive sequence presents a relatively monotonous microfaunal pattern. One must also emphasize the importance of the austral forms such as Globigerina woodi-brazieri group and Globorotalia zealandica. Certain forms illustrated by Boersma (1977), under the name of Globoquadrina sp. (spiny forms), occur in Zones N4 to N6, of the lower Miocene. They disappear in $516-30, C C$.

\section{Hole 516A}

Sixteen cores, attaining a depth of $69.3 \mathrm{~m}$ sub-bottom, correspond to Unit 1 described for Hole 516 . Planktonic microfauna are abundant, well preserved, and show very few traces of dissolution, except for those sediments belonging to the Miocene. The distribution of the different species is given in Table 5 .

The Quaternary sequence consists of the interval from mudline to the base of Core 2. The presence of Globorotalia hirsuta and Globigerinoides ruber rosea in Core 2 is the result of contamination.

The Pliocene sequence is represented between the LAD of Globoquadrina dehiscens in Sample 516A-12-3, $50-52 \mathrm{~cm}$ and the LADs of Globorotalia miocenica and G. multicamerata in Sample 516A-3,CC. Differentiation of Subzones PL5 and PL6 is difficult because of proba- 
Table 4. Distribution of planktonic foraminifers at Hole 516.

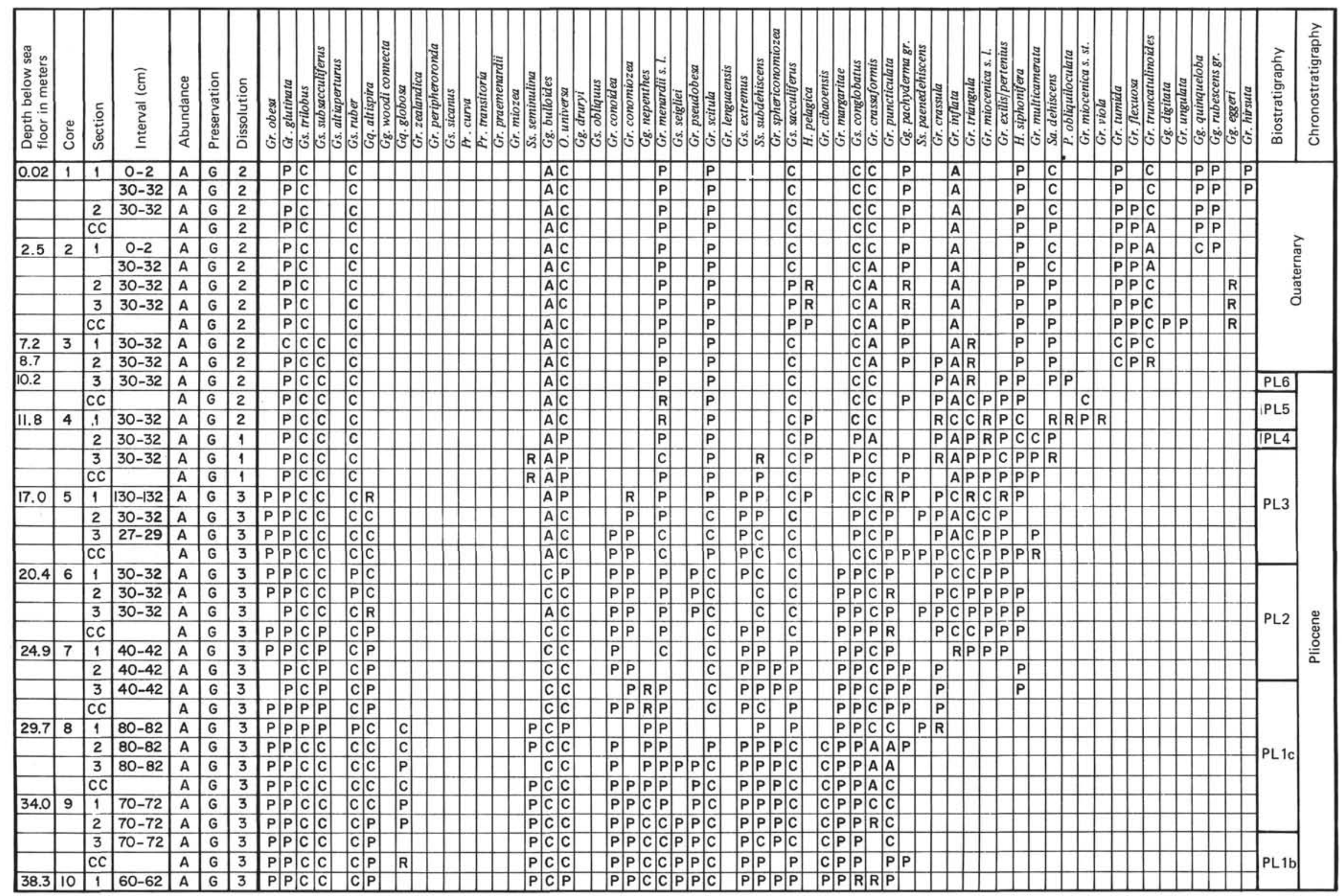

Note: Please see note on Table 2. 
Table 4. (Continued).

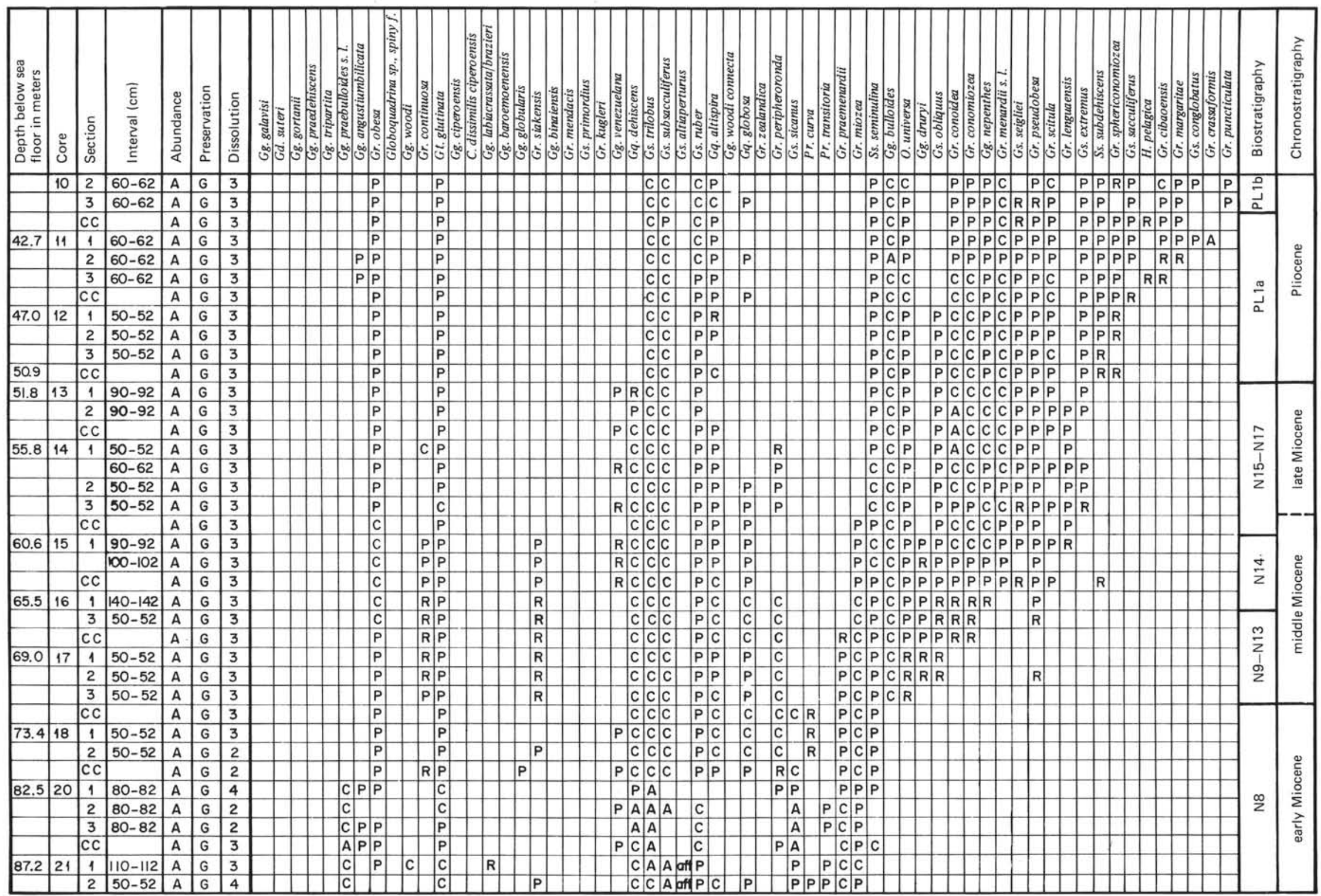


Table 4. (Continued).

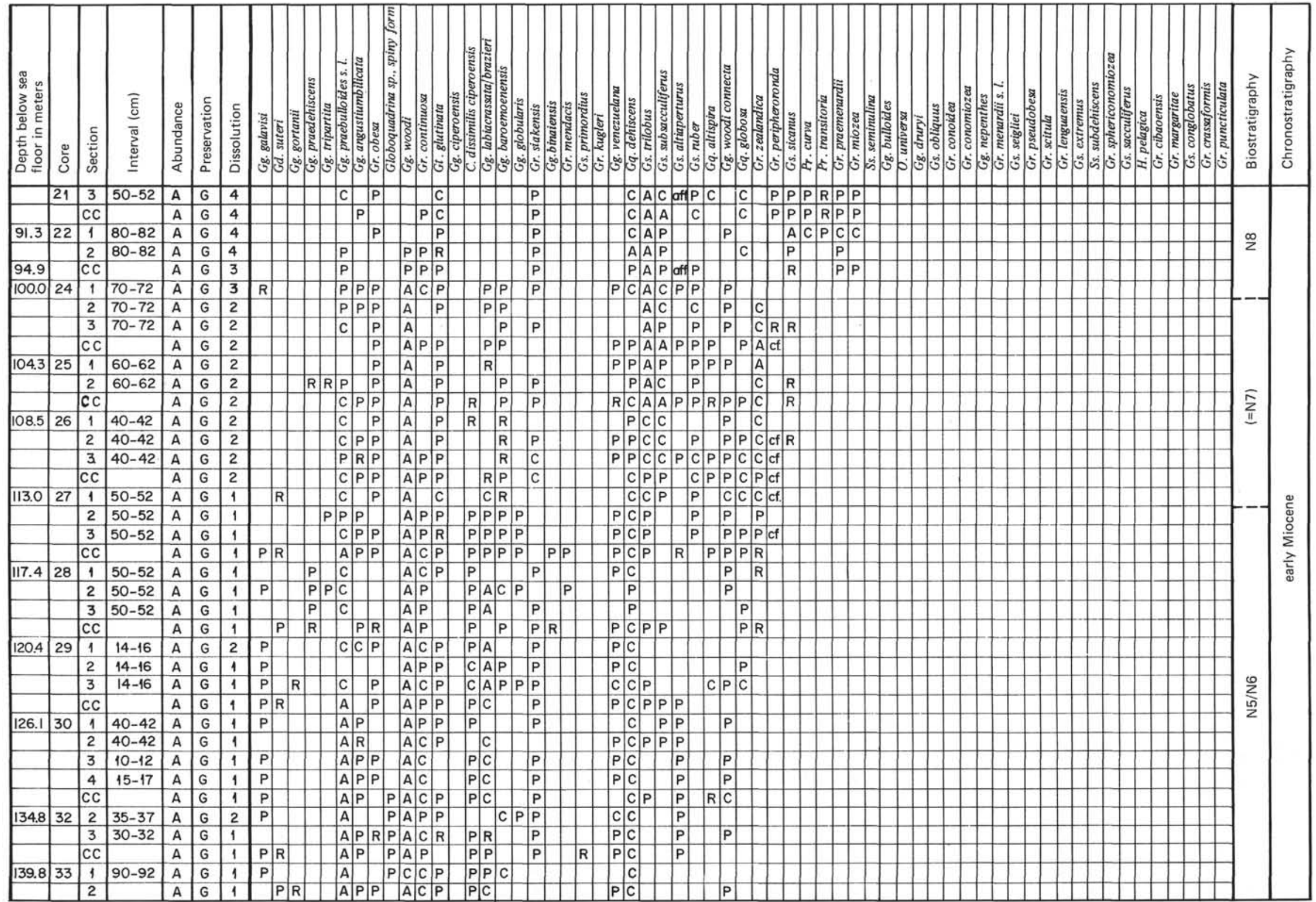


Table 4. (Continued).

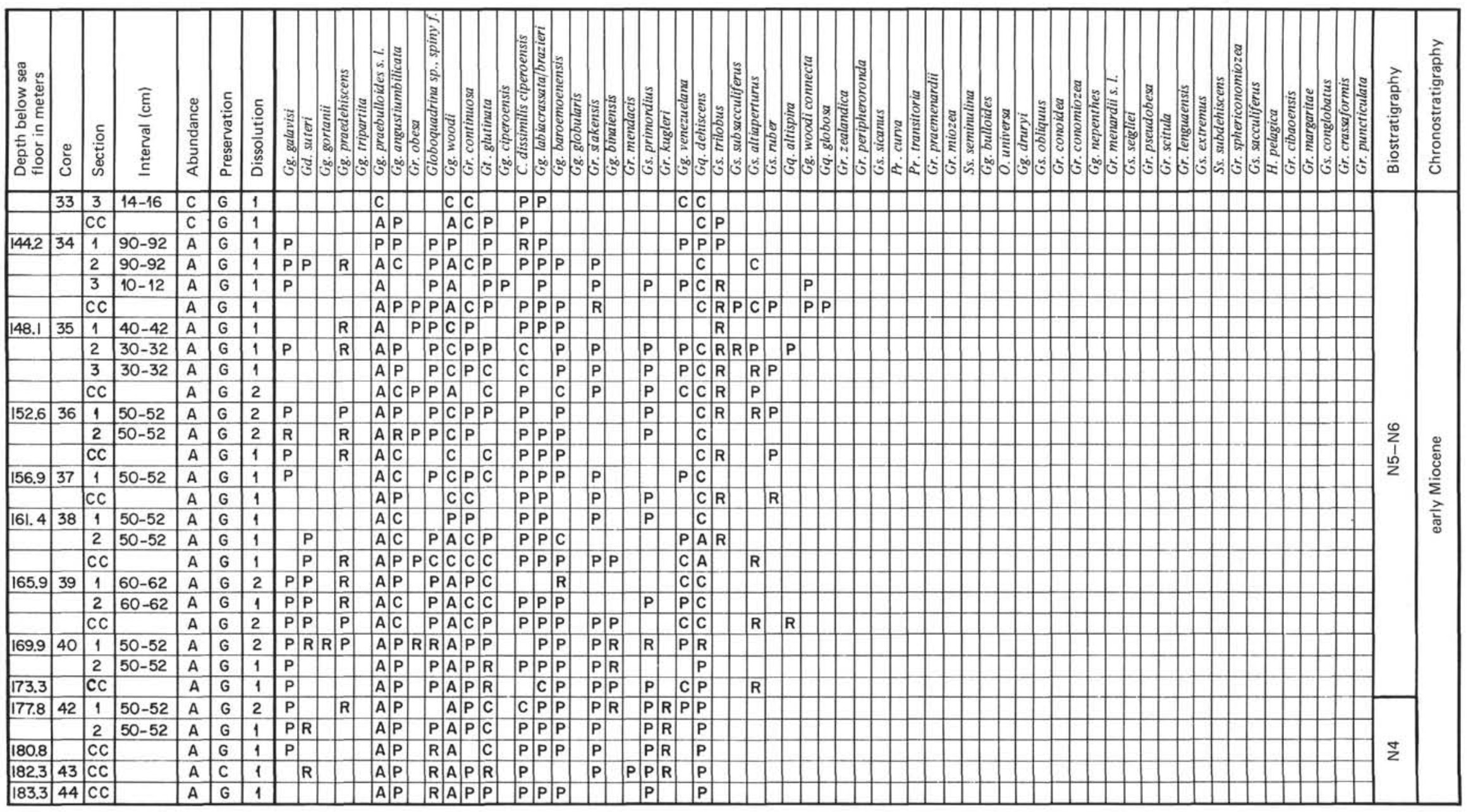


Table 5. Distribution of planktonic foraminifers at Hole 516A.

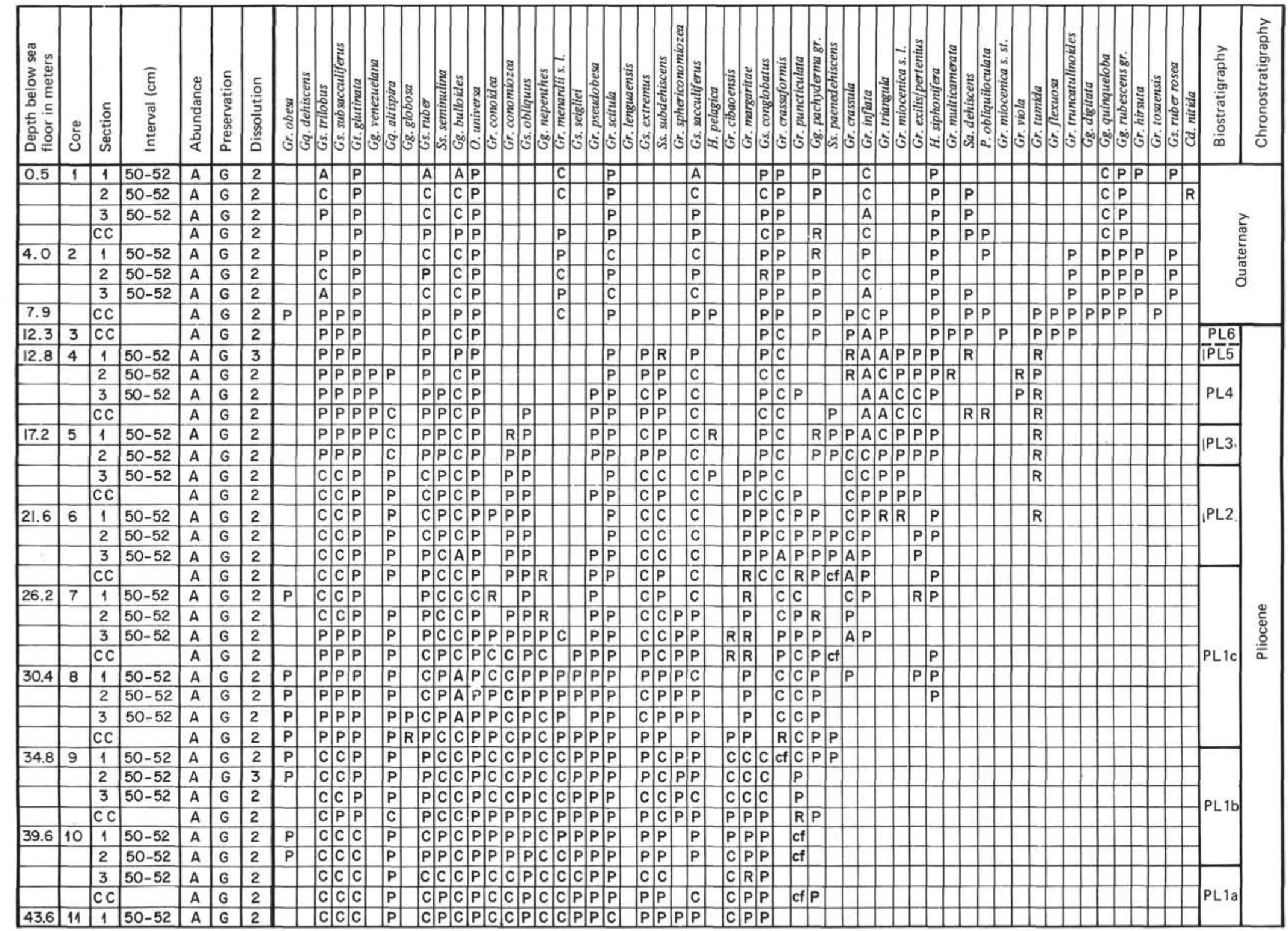

Note: Please see note on Table 2. 
Table 5. (Continued).

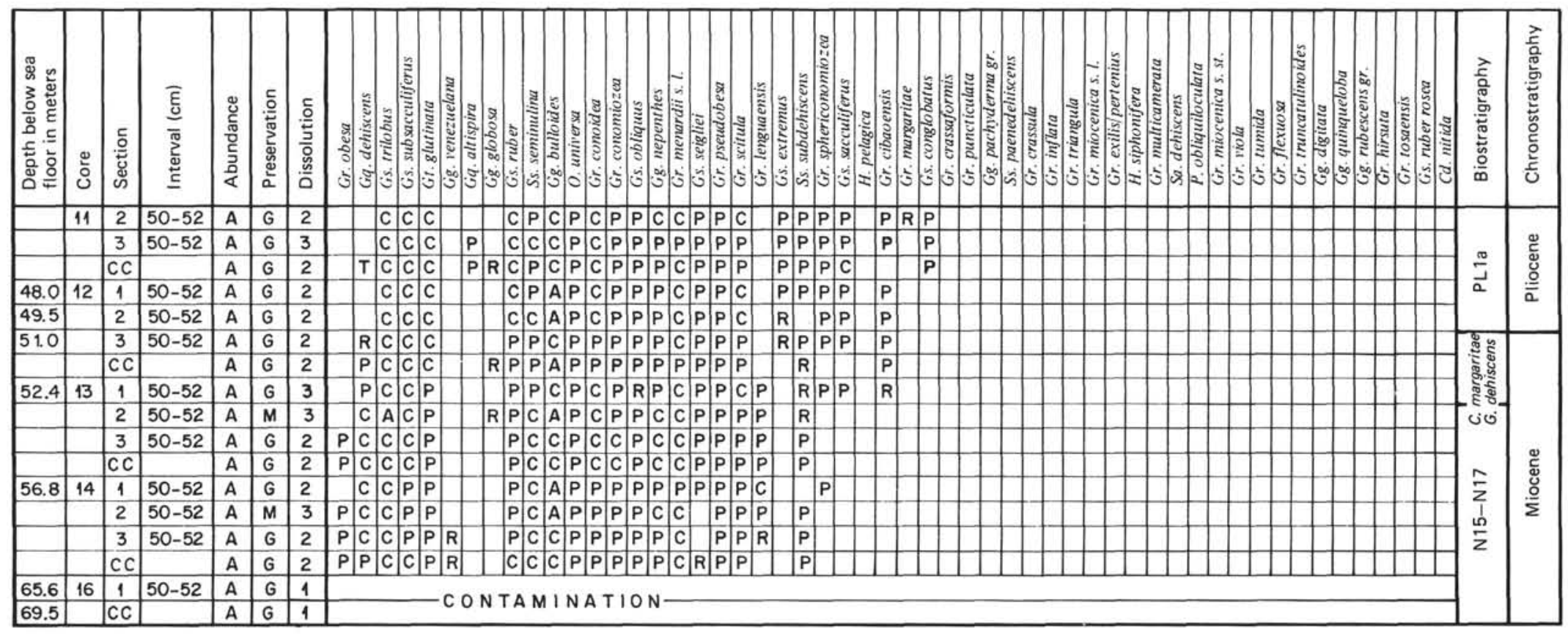


ble contamination, but the disappearance of Globorotalia exilis/G. pertenuis may indicate the PL5/PL6 boundary. Below, Biozones PL1 to PL4 are easily distinguished. The Globorotalia margaritae/Globoquadrina dehiscens Zone of the uppermost Miocene occurs between Samples 516A-13-1, 50-52 cm and 516A-12-3, 50-52 cm.

The Miocene sequence is represented in Cores 13 to 16. Contaminations in the higher zones make a precise zonal differentiation difficult, and deposits are attributed collectively to Zones N15 to N17. The most abundant and typical species are: Globorotalia conoidea, $G$. conomiozea, G. pseudobesa, and G. lenguaensis.

\section{Hole 516F}

In this hole, 128 rotary cores were recovered from the drilled interval between 169 and $1270.6 \mathrm{~m}$ sub-bottom. The drilled section consists of eight lithologic units, of which five are Cenozoic (see site chapter, Site 516, this volume). Assemblages of planktonic foraminifers are relatively well preserved; their distribution is given in Table 6. This hole begins with the lower Miocene (Zone N4). Core 1 and the top of Core 2 are assigned to Zone N5. Globorotalia kugleri disappears at the level of Sample $516 \mathrm{~F}-2-2,50-52 \mathrm{~cm}$, marking the top of Zone N4. The Oligocene/Miocene boundary is fixed at the base of Zone N4, identified at this site by the relative development of Globigerinoides primordius as the datum of its base. The first $G$. primordius are observed in Core 10, but more specimens appear in Sample 516F-5-6, 50-52 $\mathrm{cm}$ (215.1 $\mathrm{m}$ sub-bottom) nearby the occurrence of the typical and common Globorotalia kugleri.

An extensive Oligocene sequence was retrieved. The base of this sequence is defined by the extinction of the Globorotalia cerroazulensis group (Sample 516F-39,CC). The relative monotony of the planktonic foraminiferal association makes precise differentiation within the Oligocene difficult. The upper Oligocene begins at the disappearance of Pseudohastigerina sp. in Sample 516F$31-4,33-35 \mathrm{~cm}$. The LAD of Globorotalia opima opima (Sample 516F-14,CC) defines the base of Zone P22, and the first appearance datum (FAD) of Globigerina angulisuturalis (Sample 516F-24,CC) defines the base of Zone P21. Within Zone P21, Subzones P21a and P21b are differentiated on the basis of the LAD of Chiloguembelina sp. in Sample 516F-20-4, 50-52 cm. Within the lower Oligocene, however, it is possible to differentiate the various zones of Blow (1969) on the basis of his datum-taxa. According to the patterns of Stainforth and others (1975), this sequence could be assimilated within the Cassigerinella chipolensis/Pseudohastigerina micra Zone. However, because Cassigerinella is not recognized, we attribute this section collectively to Zones (P17) to (P19).

The Eocene sequence is also extensive and the microfauna common to abundant. The upper Eocene spans Core 39 to 49 . Zones P15/16 are not individualized. As for the middle Eocene, the boundaries found within Zones P10 to P14 were determined only tentatively on account of the rarity or even absence of index taxa. For instance, the scarcity of Globigerinatheka semiinvoluta sp. and absence of Orbulinoides beckmanni preclude differentiation between Zones P13 and P14.

The last occurrence of Globorotalia spinulosa marks the top of Zone P14 and the planktonic association changes slightly in Core 53, the equivalent base of this zone. These changes include the extinction of Globorotalia bullbrooki group and the beginning of Globorotalia cerroazulensis group and of Globigerina corpulenta/ Globigerinita pera. The genus Globigerinatheka $(G$. index) appears in Core 68, marking the base of Zone P11. No distinction can be made between Zones P11 and P14 because of the absence of Orbulinoides beckmanni and the short occurrence of Globorotalia aragonensis. The first fragment of Hantkenina occurs in Core 71, and the appearance of Truncorotaloides in Core 74 would generally define the beginning of Zone P10. Because of the bad to moderate preservation of this sequence, however, it is better to retain the presence of Globorotalia bullbrooki, Globigerina frontosa, and so forth in Core 77 as an element of P10. In Cores 78 and 79, a Cretaceous slump disturbs the biostratigraphic succession. So we assume that the base of P10 is probably situated in the upper part of Core 78. The Cretaceous slump prevents the recognition of a lower Eocene sequence that terminates in Core 81 and is attributed to Zone P6b.

The Paleocene sequence contains less abundant and moderately well preserved planktonic microfauna. Zones P5 and P6a are condensed in Cores 82 and 83. Globorotalia pseudomenardii (Zone $\mathrm{P} 4$ ) has a long distribution (Cores 83 to 85 ). Zone P2 is restricted to Sample 516F$88-3,55-57 \mathrm{~cm}$. Finally, Subzones P1d, P1b/c are found in Core 89, Sections 1 to 5 . In the interval $516 \mathrm{~F}-89-5$, $21-26 \mathrm{~cm}$, the fauna is badly preserved, relatively infrequent, and exclusively composed of small specimens (fraction $<150 \mu \mathrm{m}$ ). These specimens, described as EOgloberina sp., determine this short episode that could be considered as the base of the Cenozoic sequence (Globigerina eugubina Zone?). Typical Cretaceous planktonic assemblages begin at the level of Sample 516F-89-5, 28-39 cm (Weiss, this volume).

\section{SITE 517}

Twelve cores were drilled at Site 517 on the west flank of the Rio Grande Rise at a water depth of $2930 \mathrm{~m}$. Only one lithologic unit, a foraminiferal-nannofossil ooze, was recovered. Planktonic foraminifers are abundant and well preserved (Table 7). The oldest sediment was cored from a sub-bottom depth of $50.9 \mathrm{~m}$ and corresponds to the Pliocene.

The Quaternary of Site 517 is about $25 \mathrm{~m}$ thick and includes Core 517-1 to Section 517-7-1. It is the subject of a detailed study (Pujol and Duprat, this volume).

The interval between Section 517-7-2 and 517-12,CC is attributed to the Pliocene. Zone PL6 spans the level of Samples $517-7-2,50-52 \mathrm{~cm}$ to $517-8-2,50-52 \mathrm{~cm}$, where its base is determined by the disappearance of Globorotalia exilis/G. pertenuis. Zone PL5 begins with the disappearance of Globoquadrina altispira and Sphaeroidinellopsis sp. and the first appearance of Globorotalia exilis/G. pertenuis. Below, various reworked elements 
Table 6. Distribution of planktonic foraminifers at Hole 516F.

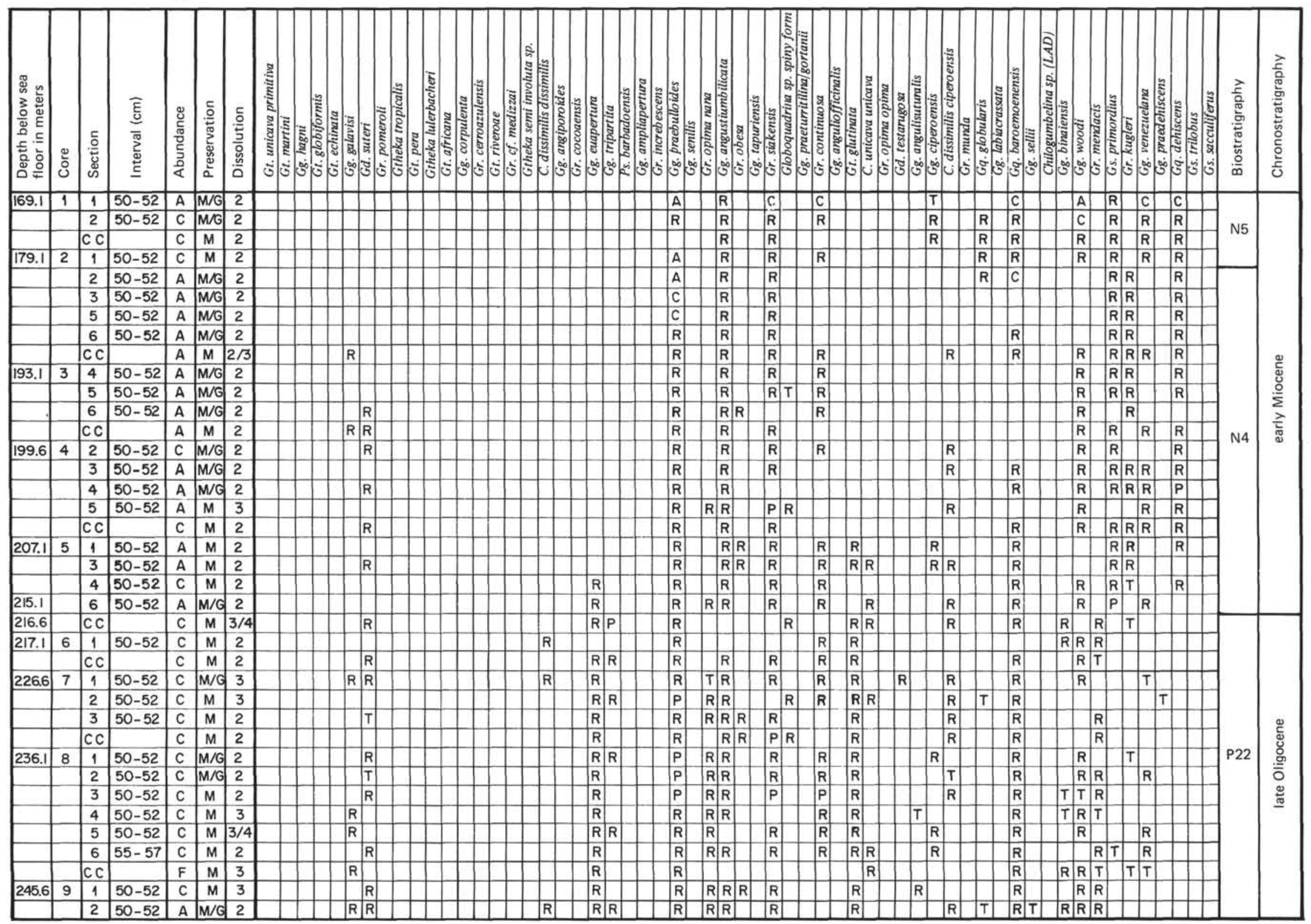

Note: Please see note on Table 2. 
Table 6. (Continued).

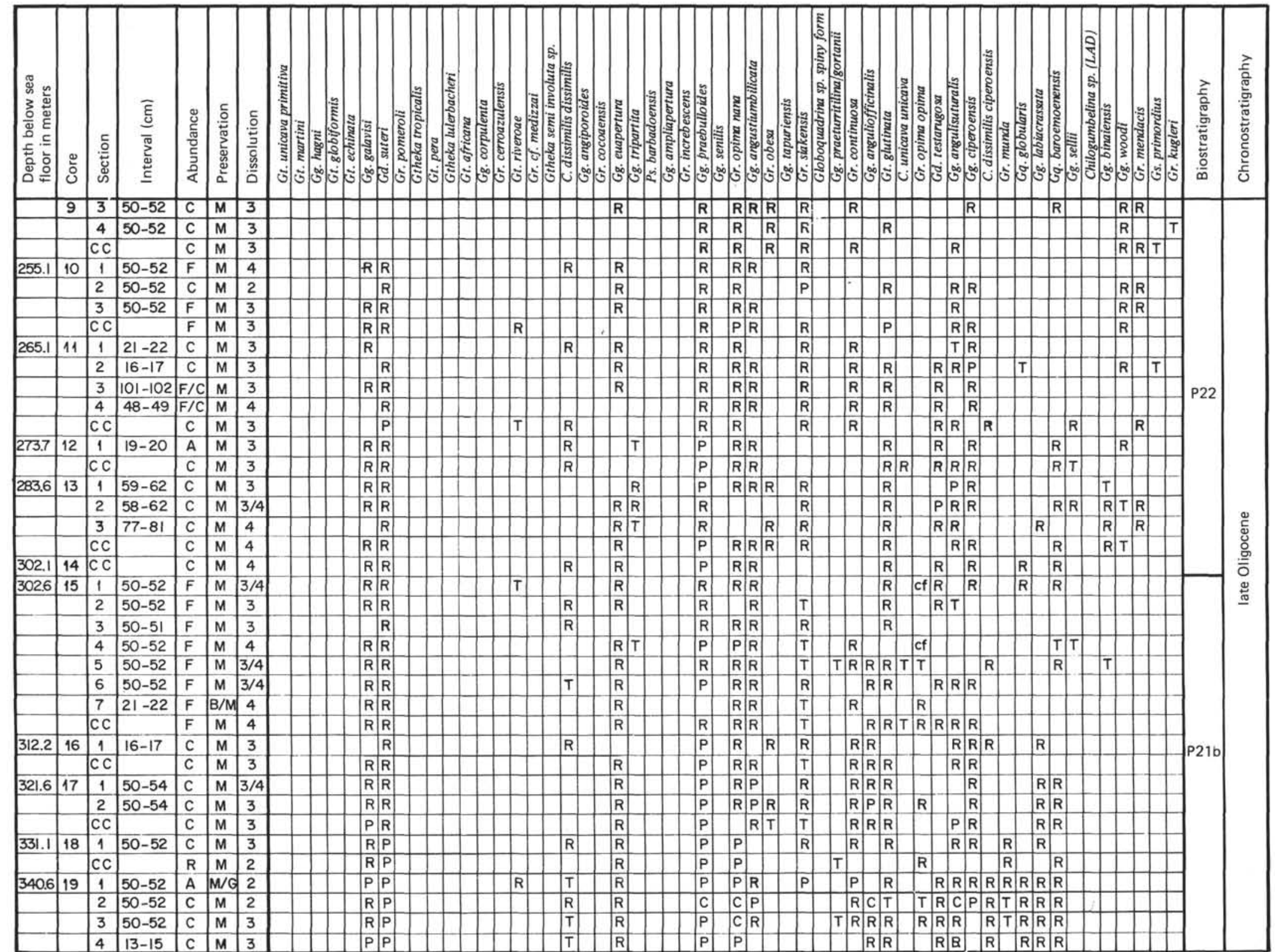




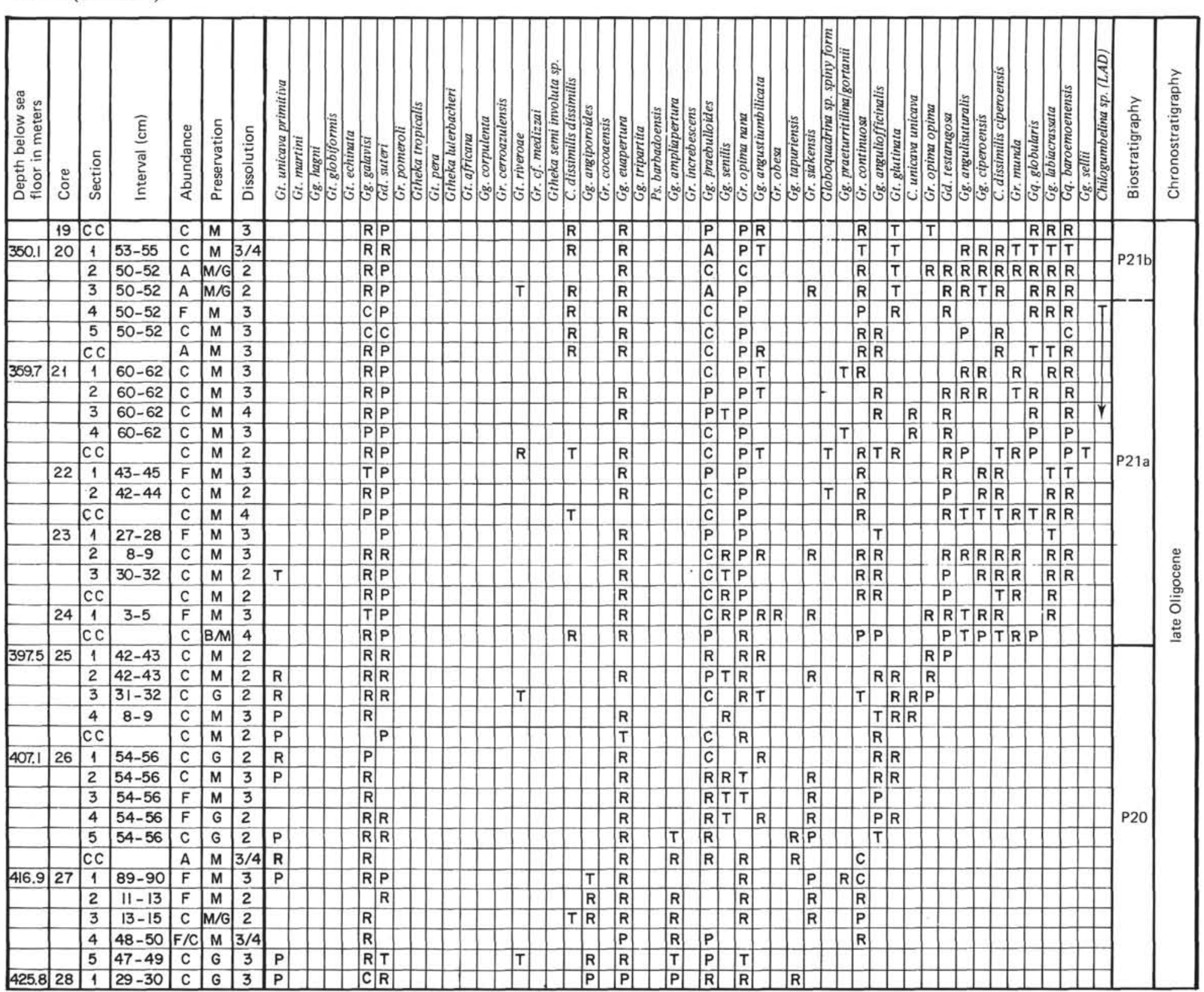


Table 6. (Continued).

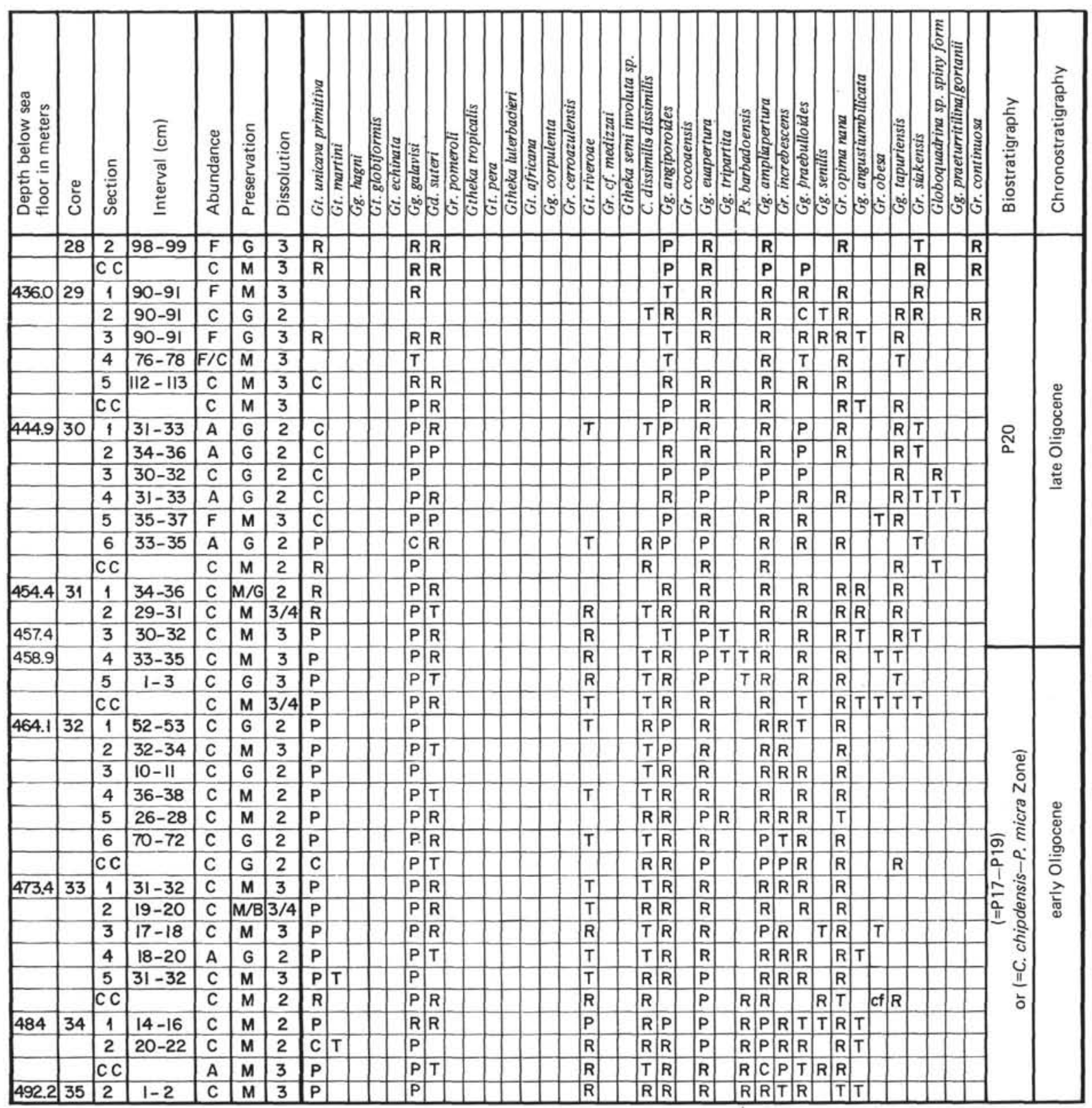


Table 6. (Continued).

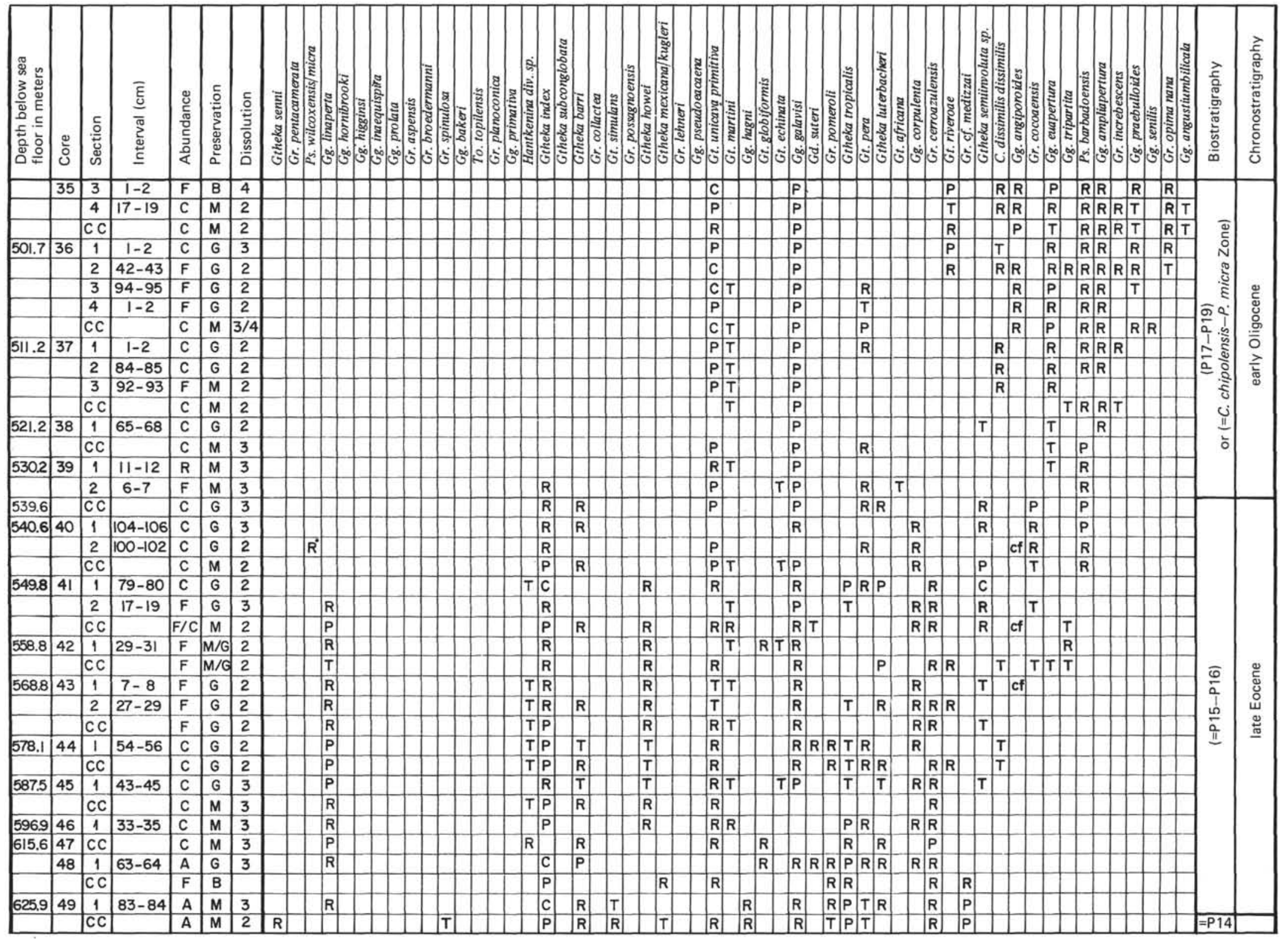


Table 6. (Continued).

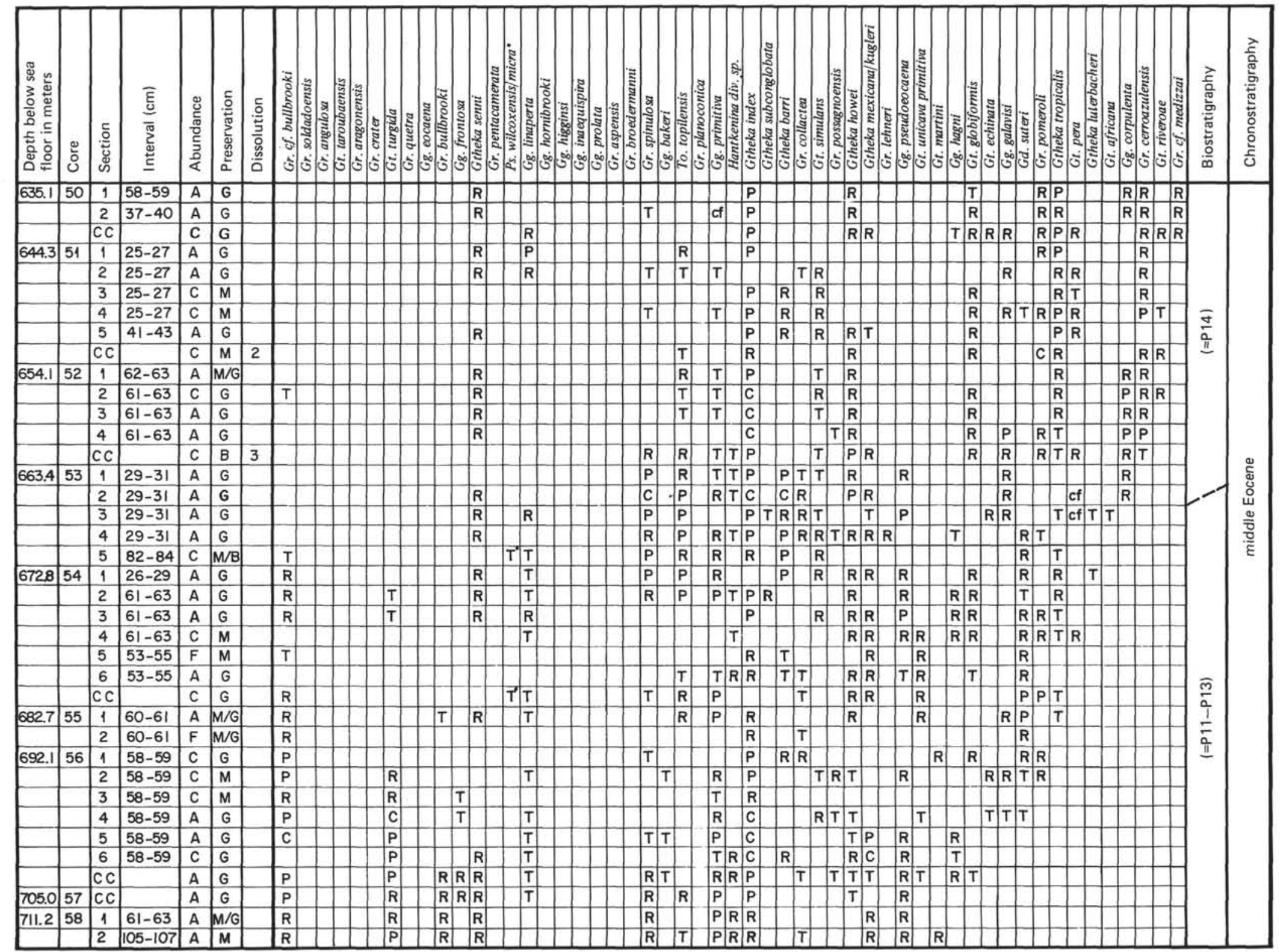


Table 6. (Continued).

\begin{tabular}{|c|c|c|c|c|c|c|c|c|c|c|c|c|c|c|c|c|c|c|c|c|c|c|c|c|c|c|c|c|c|c|c|c|c|}
\hline 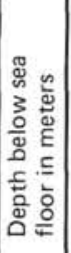 & نั & 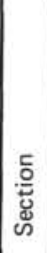 & 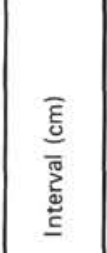 & 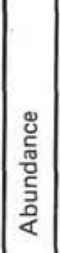 & 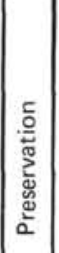 & 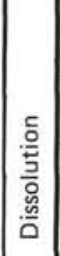 & $\mid$ & 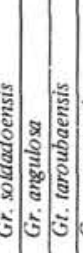 & 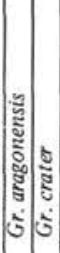 & 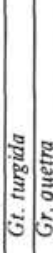 & 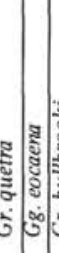 & 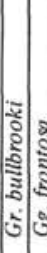 & : & 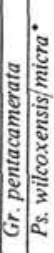 & 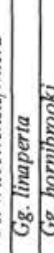 & 离 & & : & \begin{tabular}{l|l} 
\\
s. \\
:
\end{tabular} & 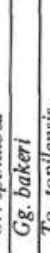 & 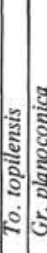 & 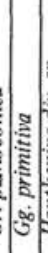 & 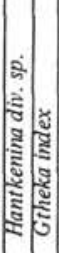 & 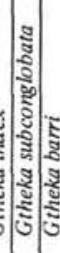 & s. & 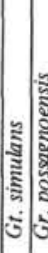 & : & 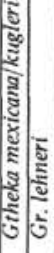 & 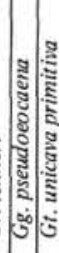 & 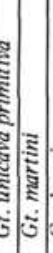 & 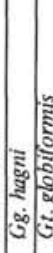 & 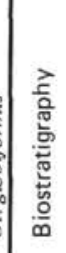 & 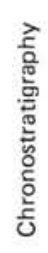 \\
\hline & 58 & 3 & $9-10$ & C & G & & \begin{tabular}{|l|}
$R$ \\
\end{tabular} & & & $\mathrm{P}$ & & R & R & & $T$ & & & & $R$ & $\mathrm{R}$ & $T$ & & $\begin{array}{ll}R & R \\
\end{array}$ & & & \begin{tabular}{|l|l|}
$R$ & \\
\end{tabular} & & $R$ & \begin{tabular}{|l|l|}
$R$ \\
\end{tabular} & & & \multirow{35}{*}{$\frac{\frac{\bar{m}}{\frac{1}{n}}}{\frac{1}{\frac{1}{n}}}$} & \multirow{38}{*}{ 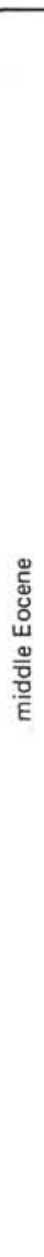 } \\
\hline & & CC & & A & $M$ & & $P$ & & & $P$ & & $R C$ & \begin{tabular}{|l|l|}
$f$ & $R$ \\
\end{tabular} & & $R$ & & & & $\mathrm{P}$ & & R & $\mathrm{P}$ & \begin{tabular}{|l|l|}
$R$ & $R$ \\
\end{tabular} & $\vec{T}$ & & \begin{tabular}{|l|l}
$T$ \\
\end{tabular} & & $\begin{array}{ll}R T \\
R\end{array}$ & $R$ & & $R$ & & \\
\hline \multirow[t]{2}{*}{717.3} & 59 & 1 & $78-80$ & $C$ & $M$ & 3 & $R$ & & & $\mathrm{P}$ & & R & & & & & & & $\mathrm{R}$ & & $T$ & $P$ & \begin{tabular}{|l|l|}
$R$ & $R$ \\
\end{tabular} & & & & $R F$ & R cf & $R$ & & & & \\
\hline & & 2 & $78-80$ & $\mathrm{~F}$ & $M$ & 3 & $\mathrm{R}$ & & & $P$ & & $R$ & & & 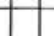 & & & & $\mathrm{R}$ & & $R$ & $R$ & $R$ & & & & $T T_{F}$ & $R$ & $\mathrm{~T}$ & 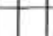 & & & \\
\hline \multirow[t]{6}{*}{721.1} & 60 & 1 & $105-107$ & $F$ & $M$ & & $R$ & & & $R$ & & & $R$ & & $T$ & & & & R & & & $P$ & $R$ & & & & & & & $R$ & & & \\
\hline & & 2 & $134-136$ & C & M & & & & & & & ( & $R$ & & $T$ & & & & $\mathrm{R}$ & & & $\mathrm{P}$ & $R$ & & & & & $R$ & 1 & & & & \\
\hline & & 3 & $110-112$ & $F$ & $M$ & & & & & $R$ & & $R$ & $R$ & & & & & & & & & & $P$ & & & $\mathrm{R}$ & $R F_{F}$ & $\begin{array}{lll}R & T\end{array}$ & & & & & \\
\hline & & 4 & $121-123$ & C & $M$ & & & & & $T$ & & $R$ & $\mathbf{R}$ & & & & & & & & & $\mathrm{P}$ & $R$ & & & $R$ & & & $R$ & & R & & \\
\hline & & 5 & $\mid 29-131$ & $F$ & $\mathrm{~m} / \mathrm{G}$ & & $P$ & & & & & & & & & & & & & & & & $R$ & & & & & & $\mathrm{R}$ & & & & \\
\hline & & 6 & $132-134$ & A & $M$ & & & & & $P$ & & $R$ & $P$ & & & & & & $R$ & & $R$ & $\mathrm{P}$ & $P$ & & & $R$ & $R F$ & $\mathrm{P}$ & & & $T$ & & \\
\hline \multirow[t]{6}{*}{729.5} & 61 & 1 & $40-41$ & R & B & $3 / 4$ & & & & $R$ & & & & & & & & & & & $\mathrm{P}$ & $\mathrm{P}$ & \begin{tabular}{|l|l}
$R$ & $R$ \\
\end{tabular} & & & & & & & & & & \\
\hline & & 2 & $27-29$ & - & - & & & & & & & & & & & & & & & & & & & & & & & & & & & & \\
\hline & & 3 & $53-55$ & - & - & & & & & & & & & & & & & & & & & & . & & & & & & & & & & \\
\hline & & 4 & $61-63$ & c & M & & C & & & & & $P$ & & & & & & & $R$ & & $R$ & $R$ & $\mathrm{R}$ & & & & & & & $T$ & $T$ & & \\
\hline & & 5 & $112-114$ & $R$ & B & & $R$ & & & $R$ & & & & & & & & & & & & & \begin{tabular}{|l|l|}
$R$ & $R$ \\
\end{tabular} & ₹ & & $\mathrm{T}$ & & $R$ & & & & & \\
\hline & & CC & & $F$ & $M$ & & $R$ & & & & & \begin{tabular}{l|l}
$R$ & $R$ \\
\end{tabular} & \begin{tabular}{|l|}
$R$ \\
\end{tabular} & & & & & & \begin{tabular}{|l|l|}
$R$ & $R$ \\
\end{tabular} & & $R$ & & $R$ & ? & & & $R$ & $T$ & $R$ & & & & \\
\hline \multirow[t]{3}{*}{738.8} & 62 & 1 & $76-78$ & A & $M$ & & $\mathrm{P}$ & & & & & & & & & $T$ & & & $R$ & $R$ & & & $\mathrm{P}$ & $P$ & & $T$ & $\mathrm{R} F$ & $P R$ & $R T$ & \begin{tabular}{|l|l|} 
& $R$ \\
\end{tabular} & \begin{tabular}{l|l}
$R$ & $T$ \\
\end{tabular} & & \\
\hline & & 2 & $69-7 \mid$ & A & $G$ & & $P$ & & & \begin{tabular}{|l|l}
$R$ & $R$ \\
\end{tabular} & R & $R R$ & $\mid \mathrm{R}$ & & & $T$ & & & $R$ & & $R$ & & $R C$ & $R$ & & $T$ & & \begin{tabular}{l|l}
$P$ & $R$ \\
\end{tabular} & $R$ & & & & \\
\hline & & 3 & $42-44$ & A & G & & $P$ & & & \begin{tabular}{l|l} 
\\
\end{tabular} & & $\begin{array}{ll}R \\
R\end{array}$ & $R$ & $T$ & $T$ & $T$ & & & & & $\mathrm{P}$ & & $\begin{array}{lll}R & C \\
\end{array}$ & $R$ & & \begin{tabular}{|l|l}
$T$ & $R$ \\
\end{tabular} & \begin{tabular}{l|l|l}
$R$ & $R$ & \\
\end{tabular} & \begin{tabular}{l|l}
$P$ & $T$
\end{tabular} & $\begin{array}{ll}R & T \\
\end{array}$ & \begin{tabular}{l|l} 
& $T$ \\
\end{tabular} & $T$ & & \\
\hline \multirow[t]{8}{*}{747.6} & 63 & 1 & $54-56$ & A & M & & $R$ & & & $R$ & & $R$ & & & $R$ & $R$ & & & \begin{tabular}{l|l}
$R$ & $R$
\end{tabular} & & $R$ & & $R R$ & $R$ & & & & $R$ & $R$ & & & & \\
\hline & & 2 & $29-3 \mid$ & A & G & & $R$ & & & $P$ & & $R$ & & & $R$ & R & & & \begin{tabular}{l|l}
$\mathrm{R}$ & $\mathrm{R}$
\end{tabular} & & $R$ & & \begin{tabular}{l|l}
$R$ & $R$ \\
\end{tabular} & $R$ & & 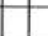 & & & $R$ & & & & \\
\hline & & 3 & $83-84$ & C & $M$ & & $R$ & & & $P$ & & $R R$ & R & & $T$ & & & & $R$ & & $R$ & & & $R$ & & \begin{tabular}{|l|l}
$R$ & $T$ \\
\end{tabular} & & $R$ & $R$ & & & & \\
\hline & & 4 & $50-52$ & $\mathrm{C} / \mathrm{A}$ & G & & $P$ & & & $P$ & & $\begin{array}{ll}R & R \\
\end{array}$ & $\mathrm{R} \mid$ & & $R$ & $R$ & & & & & \begin{tabular}{l|l}
$R$ & $R$ \\
$R$
\end{tabular} & & \begin{tabular}{l|l}
$R$ & $P$ \\
\end{tabular} & $P$ & & \begin{tabular}{|l|l}
$R$ & $R$ \\
\end{tabular} & \begin{tabular}{l|l}
$\mathrm{R}$ & $\mathrm{R}$ \\
\end{tabular} & $\mathrm{P}$ & $\mathrm{R}$ & & & & \\
\hline & & 5 & $58-60$ & C & G & & $R$ & & & $\mathrm{R}$ & & $R R$ & R & & $\mathrm{R}$ & $R$ & & & & $|T|$ & $R / R$ & & $R R$ & R & & \begin{tabular}{|l|l} 
&
\end{tabular} & \begin{tabular}{l|l}
$\mathrm{R}$ & $\mathrm{R}$ \\
\end{tabular} & R & $\mathrm{R}$ & & & & \\
\hline & & 6 & $12-14$ & A & G & & $R$ & & & $P$ & $\mathrm{R}$ & $R R$ & & & & & & & $R$ & & & & $R C$ & $c$ & $T$ & \begin{tabular}{l|l}
$T$ & $R$ \\
\end{tabular} & R $\mathrm{F}$ & R T & & & & & \\
\hline & & 7 & $14-16$ & $F$ & $M / B$ & & $R$ & & & $R$ & $R$ & $\begin{array}{ll}R & R \\
\end{array}$ & $|\mathrm{R}|$ & & & & & & & & $\mathrm{R}$ & & $R$ & $R$ & & $R$ & \begin{tabular}{l|l|}
$R$ & $R$ \\
\end{tabular} & & & & & & \\
\hline & & CC & & $R$ & G & & $R$ & & & $\mathrm{R}$ & & $R F$ & & & & $R$ & & & & & $R$ & & & $R$ & & & & & & & & & \\
\hline \multirow[t]{6}{*}{756.4} & 64 & 1 & $24-26$ & $\mathrm{~F}$ & $M$ & & & & & & & $\mid \mathrm{P}$ & $R$ & & & & & & & & & & & & & & & & & & & & \\
\hline & & 2 & $124-126$ & $R$ & $R$ & & & & & & & & & & & & & & & & & & & & & & & & & & & & \\
\hline & & 3 & $75-77$ & - & - & & & & & & & & & & & & & & & & & & & & & & & & & & & & \\
\hline & & 4 & $45-47$ & $F$ & $M / B$ & & & & & & & $\mathrm{P}$ & & & & & & & & & & & $R$ & $\mathrm{R}$ & & & & & & & & & \\
\hline & & 5 & $35-37$ & C & $M / B$ & & $R$ & & & & & $\mathrm{P}$ & $\mathrm{R}$ & & & $R$ & & & \begin{tabular}{l|l}
$P$ & $R$ \\
\end{tabular} & & \begin{tabular}{|l|l|}
$R$ & \\
\end{tabular} & & & $T$ & & & & & & & & & \\
\hline & & CC & & C & $M$ & & & & & & & \begin{tabular}{|l|l}
$P$ & \\
\end{tabular} & & & $R$ & $R$ & & & $\begin{array}{ll}\mathrm{R} \\
\mathrm{T}\end{array}$ & $R$ & $R$ & $\mid \mathrm{P}$ & & & & $T$ & & & & & & & \\
\hline \multirow[t]{2}{*}{765.9} & 65 & 1 & $80-82$ & A & $M$ & & C & & & \begin{tabular}{|l|l|}
$R$ & $R$ \\
\end{tabular} & \begin{tabular}{l|l}
$R$ & $R$ \\
\end{tabular} & $P$ & & & $\mathrm{R}$ & & & & \begin{tabular}{l|l}
$P$ & $R$ \\
\end{tabular} & $R$ & $R$ & $\mathrm{P}$ & $R$ & $R$ & & \begin{tabular}{|l|l}
$R$ & $R$
\end{tabular} & & & & & & & \\
\hline & & 2 & $97-99$ & C & M & & C & & & $\mathrm{R} R$ & $\mathrm{R} P \mathrm{P}$ & $\begin{array}{ll}C R \\
\end{array}$ & \begin{tabular}{|l|}
$\mathrm{R}$ \\
$\mathrm{R}$
\end{tabular} & & $R$ & $R$ & & & \begin{tabular}{|l|l}
$P$ & $T$ \\
\end{tabular} & & & & $T$ & $R$ & $T$ & \begin{tabular}{|l|l}
$T$ & $T$ \\
\end{tabular} & & & & & & & \\
\hline \multirow[t]{3}{*}{774.4} & 66 & 1 & $38-40$ & A & $G$ & & $P$ & & & $\mathrm{P}$ & $\mathrm{R}$ & $\mathrm{P}$ & $R$ & & $R$ & $R$ & & & $\mathrm{P}$ & & & c & & $T$ & & & & & & & & & \\
\hline & & 2 & $34-36$ & C & G & & $P$ & & & & $\mathrm{R}$ & $\mathrm{P}$ & $\mathrm{R}$ & & $R$ & & $R$ & & $\mathrm{P}$ & & & $\mathrm{P}$ & $T$ & & & & & & & & & & \\
\hline & & 3 & $47-49$ & A & G & & $\mathrm{P}$ & & & $P \mid T$ & $\begin{array}{l}\mathrm{T} \\
\mathrm{R}\end{array}$ & $R$ & & $\mathbf{R}$ & & & & & c & $R$ & & $\mid \mathbf{R}$ & $R$ & & & & & & & & & & \\
\hline
\end{tabular}


Table 6. (Continued).

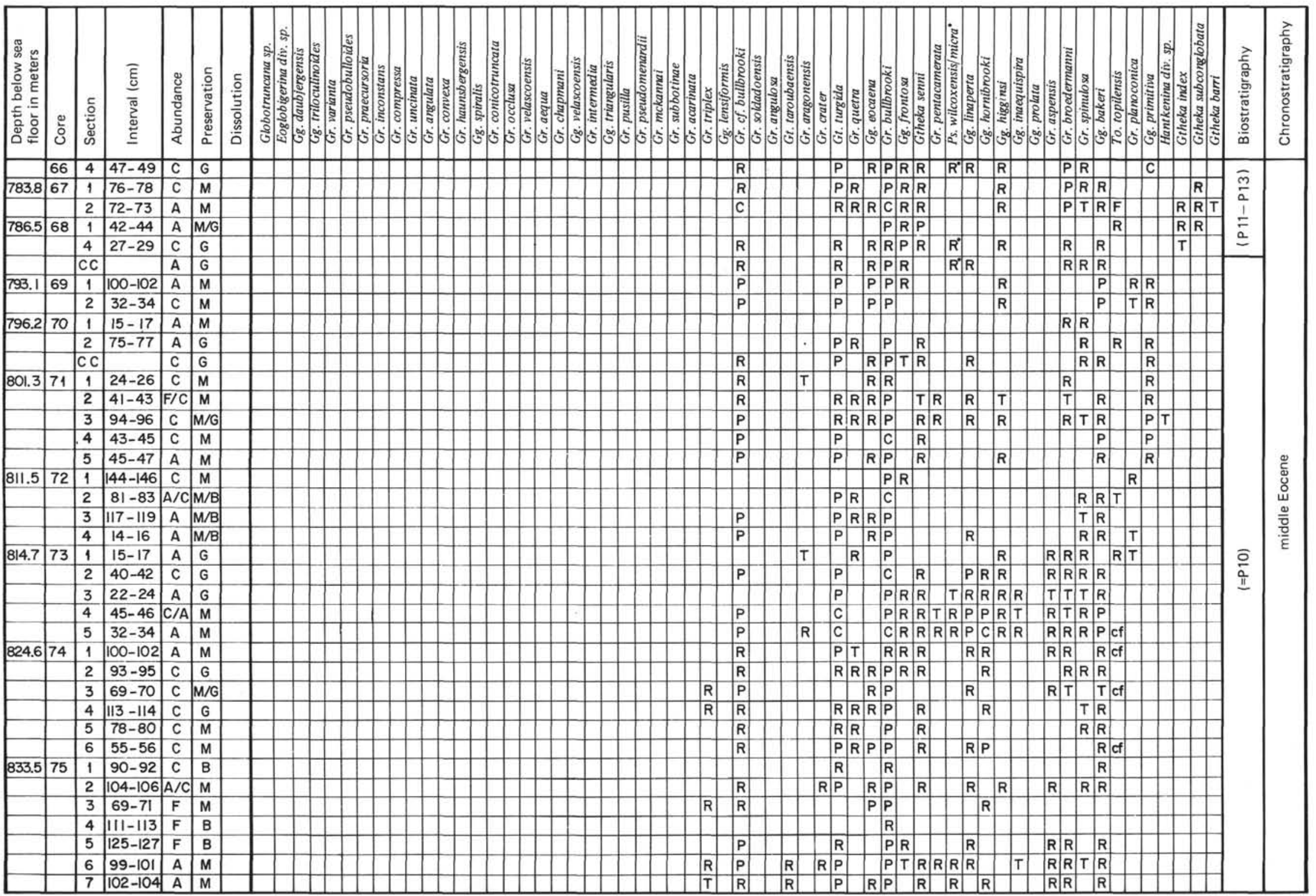


Table 6. (Continued).

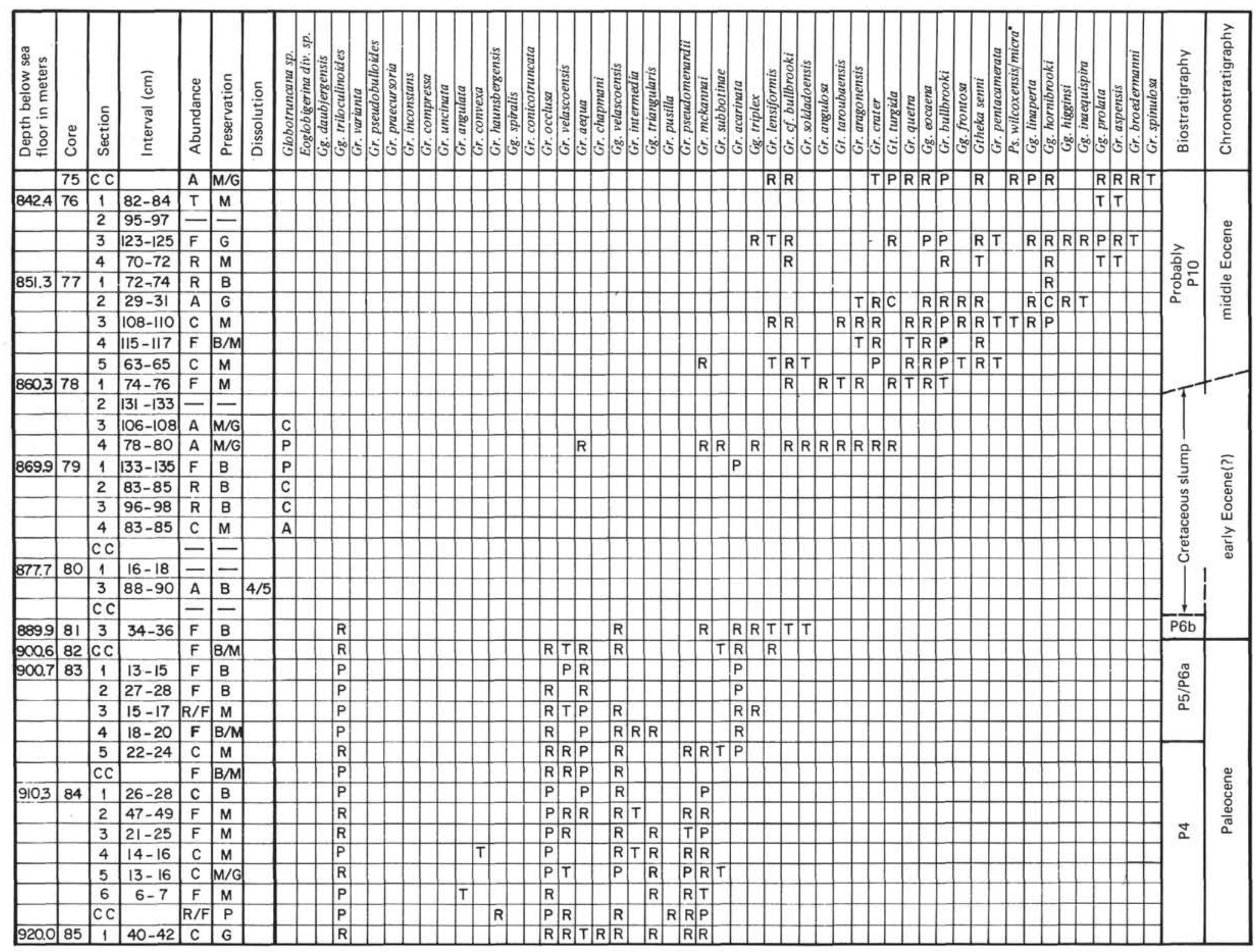


Table 6. (Continued).

\begin{tabular}{|c|c|c|c|c|c|c|c|c|c|c|c|c|c|c|c|c|c|c|c|c|c|c|c|c|c|}
\hline 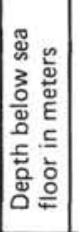 & ๖ั & 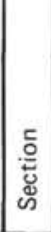 & 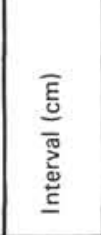 & 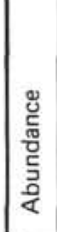 & 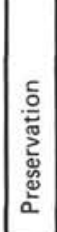 & 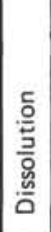 & : & 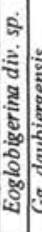 & 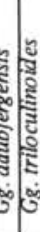 & 产 & 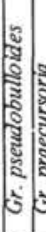 & 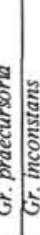 & 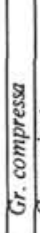 & 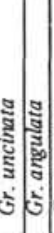 & ث: & 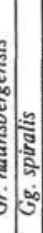 & 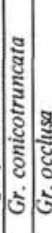 & 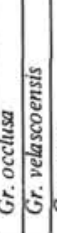 & 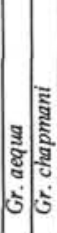 & 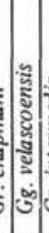 & 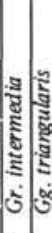 & & 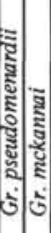 & 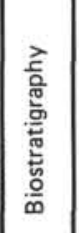 & 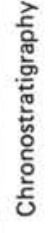 \\
\hline & 85 & 2 & $42-44$ & $C$ & $\mathrm{M} / \mathrm{G}$ & & & & P & & & & & & & & & \begin{tabular}{|l|l|}
$P$ & $R$ \\
\end{tabular} & $T$ & $R$ & R & & $R R$ & P4 & \\
\hline & & CC & & A & $M / G$ & & & & $P$ & & & & & & R & & & $P|P|$ & R & & $\mathbf{R}$ & & $\begin{array}{l}R \\
R\end{array}$ & 7 & \\
\hline 929.2 & 86 & 1 & $13-15$ & $\mathbf{R}$ & $M$ & & & & $P$ & & & & & & & & & & & $R$ & & $R$ & & \multirow{16}{*}{ P3 } & \\
\hline & & 2 & $55-56$ & $R$ & 8 & & & & 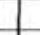 & & & & & & & & & $\mathrm{T}$ & & & & $R$ & & & \\
\hline & & 3 & $6-9$ & $R$ & B & & & & $R$ & & & & & & & & & & & & & $\mathbf{R}$ & & & \\
\hline & & 4 & $6-9$ & $F$ & MB & & & & $\mathrm{P}$ & & & & & & \begin{tabular}{|l|} 
\\
\end{tabular} & & & \begin{tabular}{l|l|}
$R$ & $T$ \\
\end{tabular} & $\mathrm{~T}$ & $R$ & & $\mathrm{P} P$ & & & \\
\hline & & 5 & $48-51$ & $R$ & $M$ & & & & $R$ & & $T$ & & & $T$ & \begin{tabular}{|l|l} 
& $\mathrm{T}$ \\
\end{tabular} & & & & & & R & ק. & & & \\
\hline & & 6 & $51-53$ & $F$ & M & & & & P & & $T$ & & & $R$ & $\mathrm{R}$ & & & $\mathbf{R}$ & & $R$ & $R$ & & & & \\
\hline & & Cc & & $\mathrm{F}$ & M & & & & P & & & & & $P$ & $R$ & & \begin{tabular}{|l|l}
$R$ & $R$ \\
\end{tabular} & $R|R|$ & $T / R$ & & $R P$ & $R$ & & & \\
\hline 939.0 & 87 & 1 & $51-52$ & $\mathrm{~F}$ & M & & & & R & & & & & $R$ & $R$ & & & $R$ & $T$ & $R$ & & & & & \\
\hline & & 2 & $57-59$ & $C$ & $M$ & & $\mathrm{~T}$ & & R & & & & & $R$ & & & & $\mathrm{P} P \mathrm{P}$ & $R$ & & & & & & \\
\hline & & 3 & $53-54$ & $F$ & $M$ & & & & P & & $R$ & & & $\mathrm{P}$ & $P$ & & \begin{tabular}{|l|l}
$R$ & $R$ \\
\end{tabular} & \begin{tabular}{l|l|}
$R$ & $R$ \\
\end{tabular} & $T R$ & 8. & & & & & \\
\hline & & 4 & $43-44$ & C & $M$ & & & & P & & $R$ & & $R$ & $P$ & \begin{tabular}{|l|l}
$P$ & $P$ \\
\end{tabular} & & \begin{tabular}{l|l}
$R$ & $R$ \\
\end{tabular} & \begin{tabular}{l|l}
$R$ & \\
\end{tabular} & & & & & & & \\
\hline & & 5 & $48-49$ & C & B & & & & $P$ & & & & $R$ & \begin{tabular}{l|l}
$P$ & \\
\end{tabular} & $\mathrm{R} P$ & & & & & & & & & & \\
\hline & & 6 & $19-20$ & C & $M$ & & & & P & & $R$ & $R R$ & $R$ & $P c f$ & $R$ & 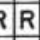 & & & & & & & & & \\
\hline & & 7 & $39-40$ & C & M & & & & P & & $R$ & R $\mathrm{R}$ & $P$ & $P$ cf & $R$ & $R$ & & & & & & & & & \\
\hline 949.0 & 88 & 1 & $93-95$ & C & $M$ & & & & P & $R$ & $\mathbf{R}$ & \begin{tabular}{l|l}
$R$ & $R$ \\
\end{tabular} & $\mathrm{P}$ & \begin{tabular}{l|l}
$R$ & \\
\end{tabular} & . P & R & & & & & & & & & \\
\hline & & 2 & $102-103$ & C & G & & & & P & & R & & $\mathrm{P}$ & $\mathrm{P}$ & \begin{tabular}{l|l}
$P$ & $R$ \\
\end{tabular} & & & & & & & & & & \\
\hline & & 3 & $55-57$ & C & $M / G$ & & & & P & $R$ & $\mathrm{P}$ & $\mathrm{R} \mid \mathrm{P}$ & & \begin{tabular}{l|l}
$\mathbf{T}$ \\
\end{tabular} & & & & & & & & & & \multirow{15}{*}{ P1d } & \\
\hline 957.7 & 89 & 1 & $15-17$ & $F$ & $M$ & & & & $\mathrm{R}$ & $R$ & $\mathrm{R}$ & $R$ & $\mathrm{P}$ & & & & & & & & & & & & 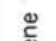 \\
\hline & & & $22-24$ & $R$ & B & & & & $R$ & & $R$ & & $R$ & & & & & & & & & & & & ষ্ঠ \\
\hline 959.2 & & 2 & $15-17$ & $F$ & $M$ & & & & $R$ & $R$ & $R$ & $R$ & $P$ & & & & & & & & & & & & $\frac{\mathscr{d}}{\pi}$ \\
\hline & & & $41-42$ & A & $M$ & & & & $P$ & $R$ & $\mathrm{P}$ & $\mathrm{R}$ & $P$ & & & & & & & & & & & & $\approx$ \\
\hline & & & $100-102$ & $F$ & $M$ & & & & $R$ & $R$ & \begin{tabular}{|l|l} 
\\
\end{tabular} & F & $P$ & & & & & & & & & & & & \\
\hline & & & $\mid 119-121$ & $A / C$ & $M / G$ & & & & $\mathrm{P}$ & $R$ & $P$ & \begin{tabular}{l|l}
$R$ & $F$ \\
\end{tabular} & $P$ & & & & & & & & & & & & \\
\hline & & & $148-150$ & $F$ & $M$ & & & & $R$ & $R$ & $\mathrm{P}$ & $\mathrm{R} F$ & $P$ & & & & & & & & & & & & \\
\hline \multirow[t]{4}{*}{961.2} & & 3 & $6-8$ & $F$ & $M$ & & & & $P$ & $R$ & \begin{tabular}{|l|l} 
& \\
\end{tabular} & $\begin{array}{l}P \\
P\end{array}$ & $P$ & & & & & & & & & & & & \\
\hline & & & $17-18$ & $F$ & $M$ & & $T$ & & $P$ & $R$ & $\mathrm{P}$ & $P R$ & $P$ & & & & & & & & & & & & \\
\hline & & & $36-38$ & $\mathrm{~F}$ & $\mathrm{M}$ & & & & $P$ & $R$ & $\mathrm{P} / \mathrm{P}$ & $\begin{array}{ll}\mathrm{R} & \mathrm{F} \\
\end{array}$ & $P$ & & & & & & & & & & & & \\
\hline & & & $83-85$ & $F$ & $M / G$ & & & & $P$ & $R$ & $R$ & \begin{tabular}{l|l}
$R$ & $F$ \\
\end{tabular} & $R$ & & & & & & & & & & & & \\
\hline \multirow[t]{6}{*}{962.6} & & 4 & $5-7$ & $R$ & B & & & & & & $R^{-1}$ & $\mathrm{~T}$ & & & & & & & & & & & & & \\
\hline & & & $38-40$ & C & $M$ & & & & $R$ & $R$ & \begin{tabular}{|l|l} 
\\
\end{tabular} & \begin{tabular}{l|l}
$P$ & $P$ \\
\end{tabular} & $\mathrm{P}$ & & & & & & & & & & & & \\
\hline & & & $68-70$ & $A / C$ & $M$ & & & & $P$ & $P$ & $\mathrm{P}$ & \begin{tabular}{l|l}
$P$ & $P$ \\
\end{tabular} & $P$ & & & & & & & & & & & & \\
\hline & & & $96-99$ & $R$ & B & & & & $T$ & & & & & & & & & & & & & & & \multirow{8}{*}{$\frac{0}{a}$} & \\
\hline & & & $132-134$ & $R$ & $M$ & & & & F & $P$ & $P$ & & & & & & & & & & & & & & \\
\hline & & & $148-150$ & $\mathrm{~F}$ & $M$ & & & & $\mathrm{~F}$ & $R$ & $P$ & & & & & & & & & & & & & & \\
\hline \multirow[t]{6}{*}{963.6} & & 5 & $0-2$ & $F$ & $M$ & & $T$ & & \begin{tabular}{l|l}
$R$ & $P$ \\
\end{tabular} & $P$ & $\mathrm{P}$ & & & & & & & & & & & & & & \\
\hline & & & $3-5$ & $R$ & B & & & & $T / P$ & $R$ & $R$ & & & & & & & & & & & & & & \\
\hline & 89 & 5 & $6-8$ & $R$ & B & & \begin{tabular}{|l|}
$T$ \\
\end{tabular} & & & & & & & & & & & & & & & & & & \\
\hline & & & $13-15$ & $R$ & $\mathrm{M} / \mathrm{G}$ & & & \begin{tabular}{l|l}
$P$ & $F$ \\
\end{tabular} & $\begin{array}{r}R \\
R\end{array}$ & & $P$ & & & & & & & & & & & & & & \\
\hline & & & $15-21$ & $F$ & M & & $R$ & \begin{tabular}{l|l}
$R$ & $F$ \\
\end{tabular} & $\begin{array}{ll}R & P \\
\end{array}$ & & P & & & & & & & & & & & & & & \\
\hline & & & $21-24$ & $F$ & $M$ & & & \begin{tabular}{l|l}
$P$ \\
\end{tabular} & & & & & & & & & & & & & & & & \multirow{2}{*}{ P1a } & \\
\hline \multirow[t]{5}{*}{963.4} & & & $24-26$ & $F$ & $M$ & & & $P$ & & & & & & & & & & & & & & & & & \\
\hline & & & $28-30$ & $\mathrm{C}$ & $M$ & & $\mathrm{R}$ & & & & & & & & & & & & & & & & & \multirow{5}{*}{\multicolumn{2}{|c|}{ Cretaceous }} \\
\hline & & & $34-36$ & $\mathrm{C}$ & $M$ & & \begin{tabular}{|l|l}
$P$ \\
\end{tabular} & & & & & & & & & & & & & & & & & & \\
\hline & & & $36-38$ & $\mathrm{~F}$ & $M$ & & $\mathrm{P}$ & & & & & & & & & & & & & & & & & & \\
\hline & & & $55-58$ & C & $M$ & & C & & & & & & & & & & & & & & & & & & \\
\hline 965.7 & & 6 & 64-65 & A & $M$ & & A & & & & & & & & & & & & & & & & & & \\
\hline
\end{tabular}


Table 7. Distribution of planktonic foraminifers at Hole 517.

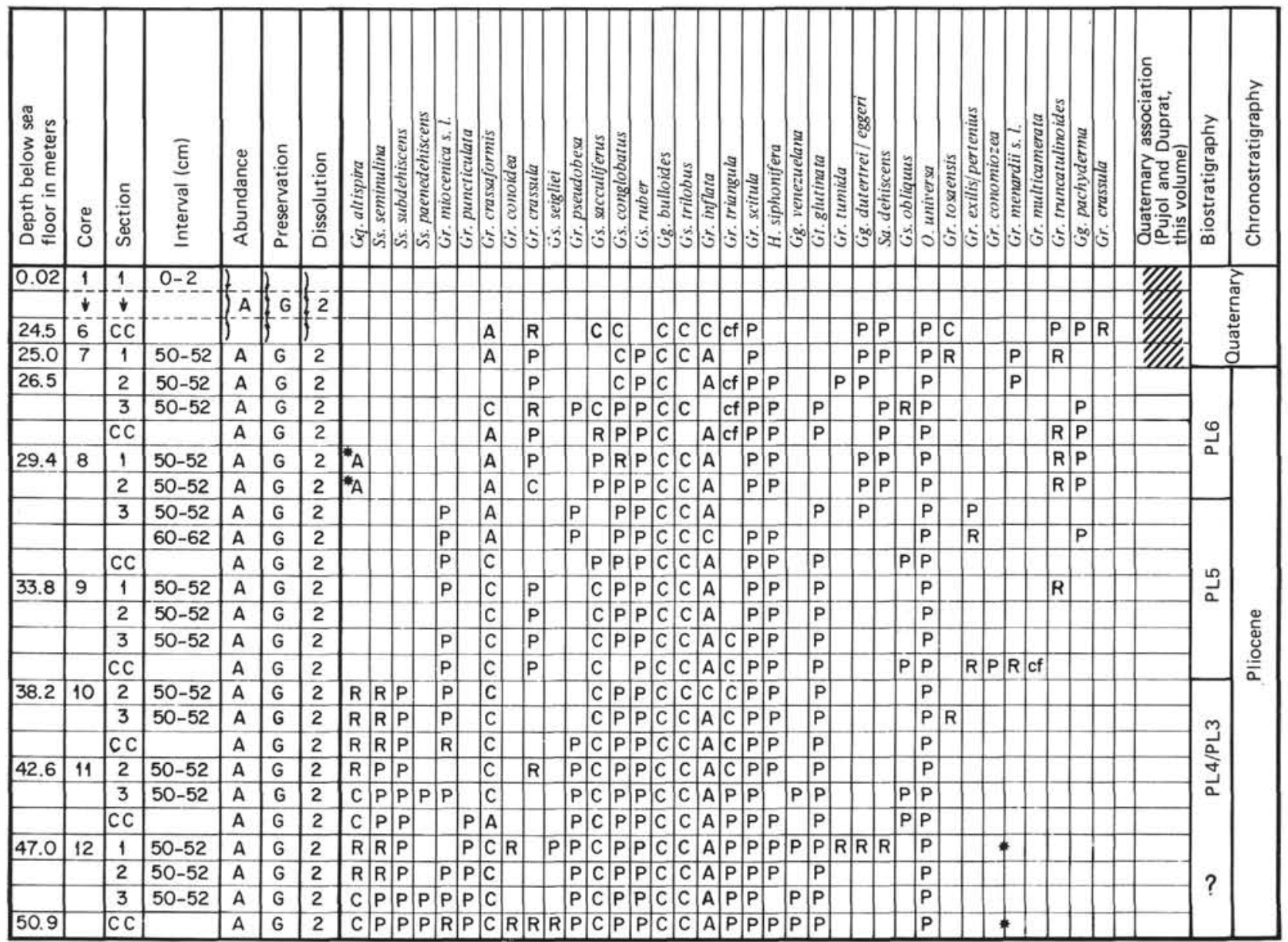

Note: Please see note on Table $2 ; \quad *$ = reworked.

or some contaminants preclude differentiation of Zones PL3 and PL4. Probably the last core from Site 517 corresponds to Zone PL2 or PL3.

\section{SITE 518}

This site is located in a water depth of $3950 \mathrm{~m}$ on the lower western flank of the Rio Grande Rise. The deepest sediment recovered came from $71.94 \mathrm{~m}$ sub-bottom in Core 18. This hole has been divided into two lithologic units: Unit 1, calcareous ooze (Cores 1 to 4 ) and Unit 2, terrigenous mud. Planktonic foraminifers from this hole are abundant, and dissolution is minor to important, especially in the deepest cores (Table 8).

This HPC hole cuts through a sequence extending from the Recent to lower Miocene with a probable hiatus. The Pliocene/Quaternary boundary is probably in Core 518-5 (17.8 m sub-bottom). Contamination or disturbances produce the overlap in the distribution of Globorotalia truncatulinoides and G. exilis/G. pertenuis. The last occurrence of Globoquadrina dehiscens marks the base of the Pliocene sequence in Sample $518-11-2,50-52 \mathrm{~cm}$. The various Pliocene Subzones
PL1 to PL6 are differentiated according to Berggren's (1973) criteria.

An occurrence of Globorotalia margaritae long before the disappearance of Globoquadrina dehiscens makes recognition of the upper Miocene $G$. dehiscens/ Globorotalia margaritae Zone possible. It occurs between Samples 518-13-1, 83-85 cm, and 518-11-2, 50-52 $\mathrm{cm}$. Cores 13 and 14 probably belong to Zone N17. Between Cores 14 and 15, a radical microfaunal change occurs. The deposits of Cores 15 and 16 show strong dissolution effects and Pliocene-Pleistocene contamination, preventing a reasonable biostratigraphic identification. Core 17 presents a new, abundant, and better preserved microfauna, including Globorotalia siakensis, Globorotalia zealandica and, at the base, Praeorbulina transitoria, Globorotalia praemenardii. This association can probably be assigned to the base of Zone N9. Finally, Core 18 contains a very dissolved microfauna where only the most resistant specimens remain (Globorotalia miozea, Sphaeroidinellopsis seminulina, and Catapsydrax sp.). This association could represent the lower Miocene deposits (probably Zone N6). 
Table 8. Distribution of planktonic foraminifers at Hole 518.

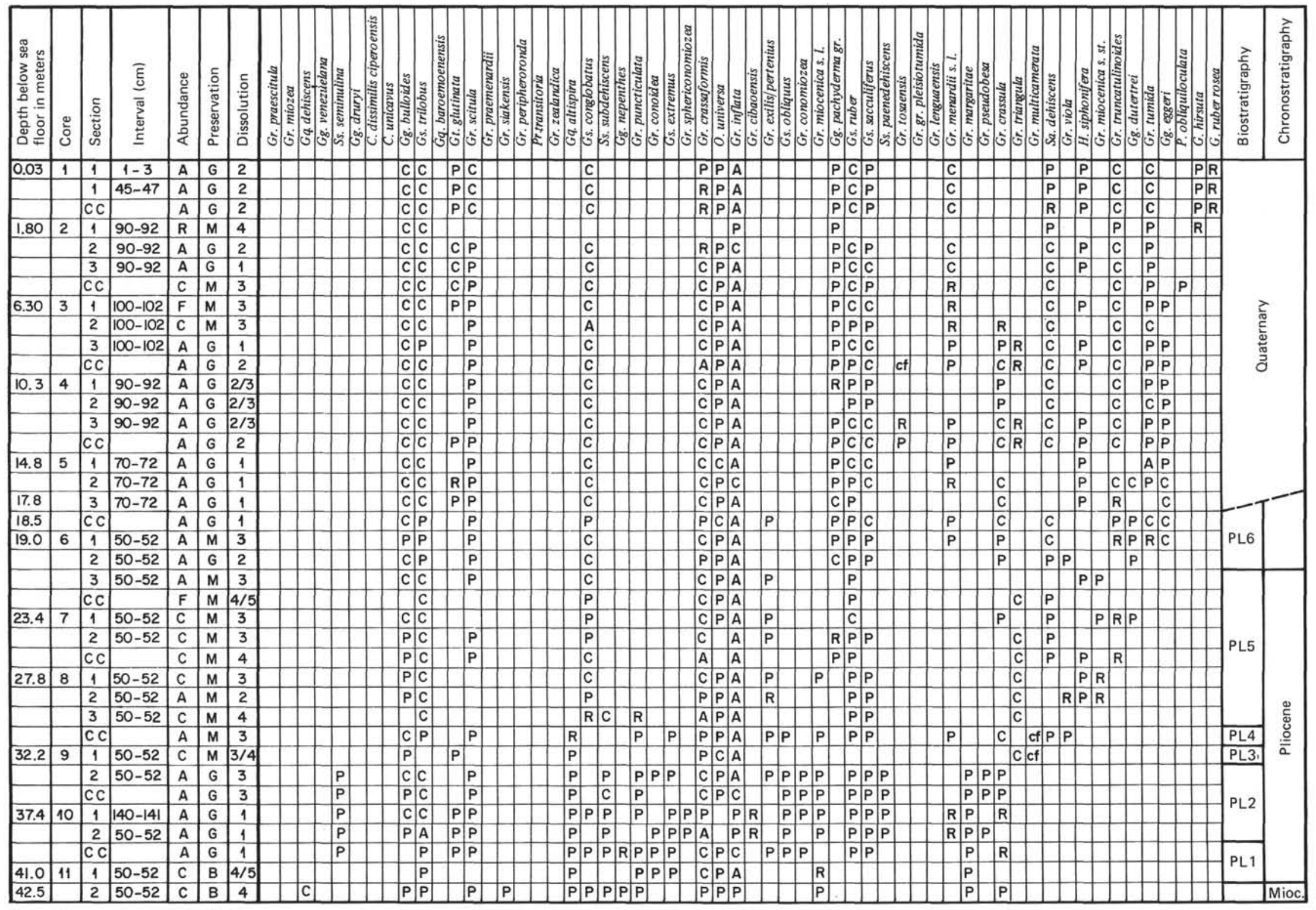


Table 8. (Continued).

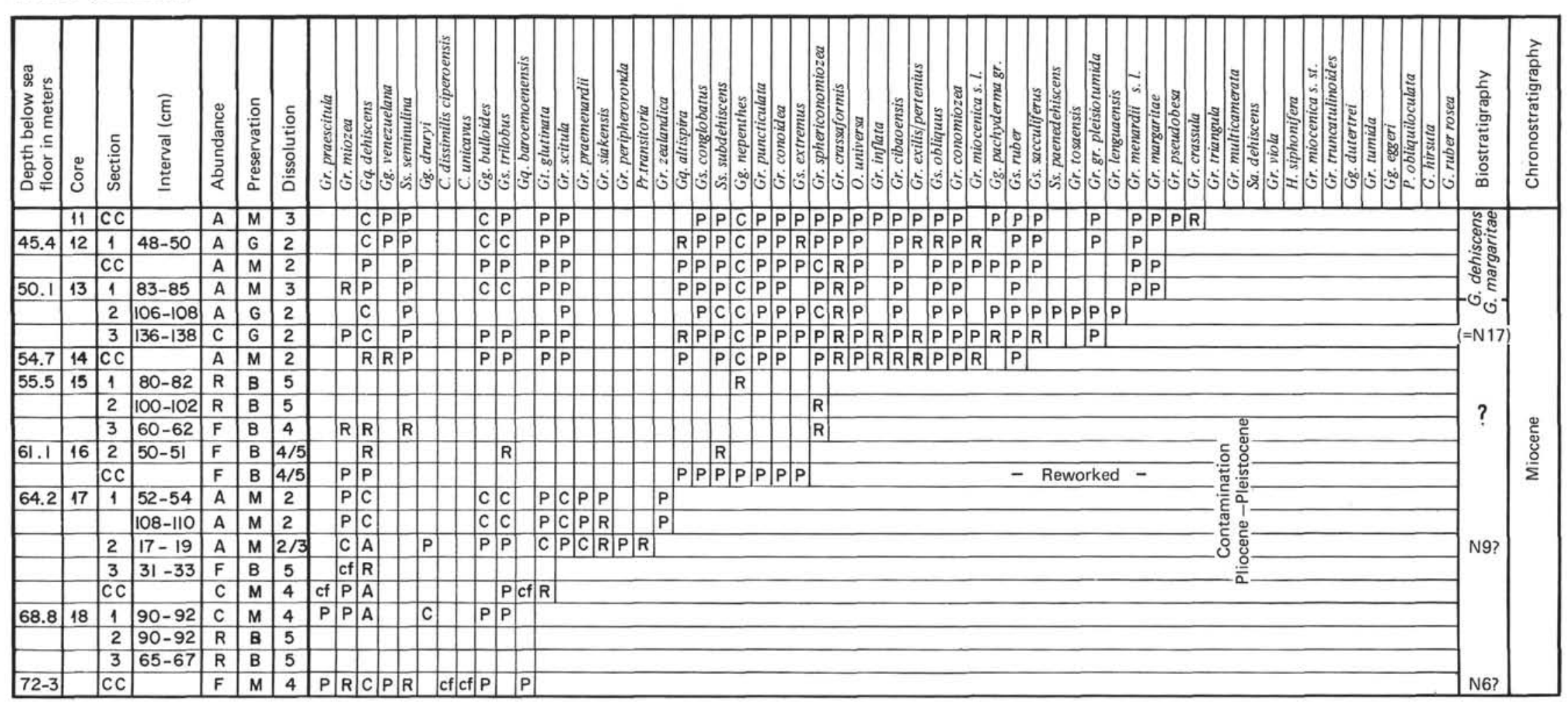




\section{CENOZOIC ZONES AND BOUNDARIES}

\section{Quaternary}

Quaternary deposits were cored with HPC in the four sites (Holes 515, 515A, 516, 516A, 517, and 518), and the Quaternary sequence of Site 517 was studied in detail (Pujol and Duprat, this volume).

It is difficult to evaluate exactly the importance of the Quaternary series at these different sites, because the most recent deposits have been reworked either by bottom currents or by the coring method. The most recent deposits recovered are marked by the presence of rightcoiling Globorotalia hirsuta and Globigerinoides ruber rosea. The Pliocene/Pleistocene boundary is determined by the appearance of Globorotalia truncatulinoides and, given the intensive dissolution of planktonic microfauna at Site 515 , is difficult to spot. The Quaternary is certainly thicker than $50 \mathrm{~m}$ at Holes 515 and 515A, amounts to about $8.7 \mathrm{~m}$ at Site 516, $26.5 \mathrm{~m}$ at Site 517, and about $18 \mathrm{~m}$ at Site 518 .

\section{Pliocene}

Difficulties arise at the Pliocene/Quaternary boundary in the sites studied, because the extinction of Globigerinoides obliquus was not observed throughout. In most of the Leg 72 sites, the first occurrence of Globorotalia truncatulinoides or the disappearance of $G$. exilis/G. pertenuis determines the top of the Pliocene. In Hole 516A, the boundary is located at the disappearance of $G$. multicamerata because of the probable contamination in the underlying levels. According to Berggren (1977b), the base of the Pliocene is located at the disappearance of Globoquadrina dehiscens and Globorotalia mediterranea. Only the first of these two is defined in this study. The Pliocene is about $41 \mathrm{~m}$ thick in both Holes 516 and $516 \mathrm{~A}$, where the base is better differentiated; that is about $24 \mathrm{~m}$ thicker than in Site 518 .

\section{Miocene}

An increase in abundance of Globigerinoides primordius defines the base, and the disappearance of Globoquadrina dehiscens the top of the Miocene sequence recovered at Sites 515, 516, and 518. The extent of Miocene deposits at Site 515 is difficult to estimate because of the uncertainty in placement of the lower boundary. The most complete Cenozoic succession is in Hole 516. Site 517 did not reach to Miocene, but Miocene sediments were recovered in seven cores from Site 518.

Globorotalia conoidea and $G$. conomiozea are the most frequent and typical species in upper Miocene faunal assemblage at Holes 516, 516A, 517, and 518. We were also able to distinguish the same biozones found at Site 357 (Boersma, 1977; Berggren, 1977a) and described from piston cores of the Rio Grande Rise (Berggren, 1977b). The Globorotalia margaritae/Globoquadrina dehiscens Zone is without doubt recovered in Site 518, but we have not observed the concurrent range of these two species in Holes 516 and 516A. At Site 516, Globorotalia margaritae occurs a few samples above the $\mathrm{LAD}$ of Globoquadrina dehiscens. The Globorotalia conomiozea/G. mediterranea and $G$. miozea/G. conoidea
Zones were not distinguished and are grouped in Zone N17, according to Berggren's (1977b) assumptions. Moreover, the upper Miocene was not distinctly differentiated but recognized as Zones N15 (part)-N17.

The middle Miocene contains the first appearance of Orbulina universa. The lower boundary at Site 516 occurs in deposits where dissolution is significant; indeed, the first occurrence of $O$. universa (Sample 516-17-3, $50-52 \mathrm{~cm}$ ) is probably delayed artificially by dissolution. Only in Hole 516 is it possible to observe the last occurrence of $G$. siakensis and the first occurrence of $G$. nepenthes, species that define Zone N14. Although G. lenguaensis is well represented at the top of N14, the subsequent Zones N9 to N13 are not differentiated.

The lower Miocene is distinct in Holes 516 and $516 \mathrm{~F}$. The Globigerinoides sicanus occurrence clearly determines the base of Zone N8. The disappearance of Catapsydrax dissimilis helps locate the top of N6. Unfortunately, the absence of Globigerinatella insueta in the deposits does not identify the boundary between Zones N6 and N5. An increase in abundance but not first appearance of Globigerinoides primordius determines the base of Zone N4. This development occurs near the first appearance of Globorotalia kugleri s.s.

The Miocene sequence is characterized by a temperate association also recorded in southeastern Australia (Jenkins, 1960); New Zealand (Jenkins, 1971; Jenkins, 1975); and in the southeastern Atlantic (Jenkins, 1978). Characteristic is the development of the Globigerina labiacrassata-brazieri-woodi group, which spans the entire lower Miocene. The genus Globoquadrina is also well represented, in particular the species $G$. baroemoenensis, which disappears at the top of Zone N7. G. altispira is common only at the top of Zone N6. One of the most characteristic lower Miocene species of Site 516 is Globorotalia zealandica s.1. This population has a short stratigraphic occurrence limited to Zone N7 and the top of Zone N6. Some specimens of $G$. praescitula (not included in Table 4) are noted together with the $G$. zealandica group from the top of Zone N6. Finally, several specimens may be compared to those forms illustrated by Boersma (1977), for example Globoquadrina sp. spiny form. These forms occur at the base of the Miocene and disappear in the bases of Zones N5 and N6, before the appearance of Globorotalia zealandica.

If the different holes of Site 516 are considered, the Miocene as a whole represents a thickness of almost $150 \mathrm{~m}$. The lower Miocene is the best represented sequence (about $130 \mathrm{~m}$ ).

\section{Oligocene}

The Oligocene was found on the basis of planktonic microfauna, but only in Hole 516F. Its upper boundary corresponds to the Globigerinoides primordius increase in abundance and its base to the disappearance of the forms belonging to the Globorotalia cerroazulensis group.

The upper Oligocene accounts for about $242 \mathrm{~m}$ of Hole $516 \mathrm{~F}$ and its microfaunal association, despite its low diversity, contains the main index taxa. In particular Globorotalia testarugosa, well represented in Zone 
P21 and in the base of Zone P22, is also found in the Austral area (Jenkins, 1971) and in the southeastern Atlantic (Toumarkine, 1978). This species occurs frequently as do Globigerina angulisuturalis and the first Globoquadrina. Globoquadrina sp. (spiny form) persists throughout the upper Oligocene Zone in P20. Globigerina gortanii, generally thought to first occur in Zone P17, is observed here for the first time further upsection in Zone P20. A few specimens of G. sellii are observed in Zone P22, and the first ones appear in P21. Finally, the upper Oligocene sequence is marked by a drastic increase in abundance of $G$. galavisi and $G$. praebulloides. G. ampliapertura is mostly represented in the Zone P20 and below.

The lower Oligocene was not subdivided because of the lack of standard species for Blow's (1969) zones or because of their unusual succession in relation to the author's pattern. Taking into account the definition of the upper and lower boundary at the lower Oligocene, we could attribute this sequence to the Cassigerinella chipolensis/Pseudohastigerina micra Zone of Stainforth and others (1975); however, the absence of C. chipolensis does not permit identification. The lower Oligocene of Hole $516 \mathrm{~F}$ probably corresponds to Zones P17 (part) to P19. G. tapuriensis is observed only in the top of the sequence (Sample 516F-33,CC), Globorotalia increbescens disappears near the LAD of Pseudohastigerina. The typical Globigerina angiporoides begins in the middle of this section, at the same place that G. ampliapertura and G. euapertura appear. Together, the upper and lower Oligocene sections are more than $330 \mathrm{~m}$ thick.

\section{Eocene}

The Eocene/Oligocene boundary is marked by the last appearance of few Globigerinatheka species and Globorotalia cocoaensis. The upper Eocene (Zones P15 to P16) is differentiated on the basis of the last appearance of the keeled Globorotalia, G. spinulosa. No biozones are distinguished within the upper Eocene. The genus Globigerinatheka is developed with $G$. index, $G$. luterbacheri, and mostly $G$. semiinvoluta $\mathrm{sp}$. This last species is limited to this interval but it does not appear as typical. It could well be compared with Toumarkine's figures of species recognized from the southeastern Atlantic $(1978, \mathrm{pl} .5$, figs. $15-21)$. So, it is probably not a reliable datum species for this area.

Orbulinoides beckmannii is not observed; therefore no differentiation between middle Eocene Zones P11 to P14 can be made on the basis of this generally used datum. The first appearance of Globigerinatheka $(G$. index) marks Zone P11. Moreover, Globorotalia aragonensis has a short occurrence in this sequence between Zones P11 and P14, and its last appearance in Core $516 \mathrm{~F}-71$ seems to be too low in a biostratigraphic sequence.

Zones below P10 are difficult to distinguish. The first fragments of Hantkenina appear in Core 516F-71, but because of the moderate to bad preservation of the samples it seems better to retain another datum for the definition of the base of Zone P10, namely the first appear- ance of Truncorotaloides sp. in Sample 516F-74-6, $55-56 \mathrm{~cm}$. Just above the thick slump of Cretaceous sediment, typical Globorotalia bullbrooki occurs in Core $516 \mathrm{~F}-78$, suggesting a middle Eocene age for the sediments. So the base of Zone P10 is probably in or below Core 516F-78. The base of the Eocene, Zone P6b, occurs in Core 516F-81. The total thickness of the Eocene in Hole $516 \mathrm{~F}$ is difficult to establish, because of a Cretaceous slump of at least $10 \mathrm{~m}$. This slump disturbs the biostratigraphic succession between Cores 516F-79 and 516F-78.

We can suppose, however, that the Eocene sequence has a thickness of about $20 \mathrm{~m}$ for the lower Eocene, 240 $\mathrm{m}$ for the middle Eocene, and about $60 \mathrm{~m}$ for the upper Eocene. In contrast to Hole $516 \mathrm{~F}$, a complete sequence of lower Eocene occurs in Hole 515B. Zones P6b to P9 may be differentiated despite unfavorable conditions of preservation. In this case, thickness of the lower Eocene is about $15 \mathrm{~m}$.

\section{Paleocene}

A Paleocene series about $100 \mathrm{~m}$ thick occurs in Hole $516 \mathrm{~F}$. We succeeded in distinguishing Zones P1 to P6a. The faunal association recovered does not show any peculiarities. The main indices are observed, and their distribution corresponds to patterns already established. Subzones P1b and P1c are not differentiated because of the low representation of the Globigerina daubjergensis population and the appearance of the G. pseudobulloides population.

A short interval of about $5 \mathrm{~cm}$ near $963 \mathrm{~m}$ sub-bottom contains only small planktonic foraminifers in the fraction between 60 and 150 microns. This microfauna is often badly preserved and corresponds to Eoglobigerina sp. (Plate 1, Figs. 1-3).

It does not seem possible to assign this episode to the typical G. eugubina Zone because of the nondetermination of the standard species. Its place, however, in the stratigraphic succession and its fauna may lead us to assume that this is a Paleocene Subzone P1a.

\section{TAXONOMIC NOTES}

The taxonomic concepts and terminology of Blow $(1969,1979)$, Berggren (1977b), and Stainforth and others (1975) are used herein, wherever possible. This study aimed to solve mostly stratigraphic problems. Therefore, the illustration of the most significant species of the southwestern Atlantic are given in the order of stratigraphic appearance. For reading convenience, however, the comments and the list of the illustrated species are arranged in alphabetic order.

Catapsydrax dissimilis ciperoensis (Blow and Banner, 1962). Plate 14, Figures 9-10.

Catapsydrax dissimilis dissimilis (Cushman and Bermudez, 1937). Plate 12, Figure 5.

Catapsydrax unicava primitiva (Blow and Banner, 1962). Plate 10, Figure 7.

Eoglobigerina sp. Plate 1, Figures 1-3. The subgenus of Morozova (1959) is retained here to differentiate several little forms limited to P1a Zone.

Globigerina ampliapertura Bolli, 1957. Plate 10, Figures 4, 8, 10. Globigerina angiporoides Hornibrook, 1965. Plate 11, Figure 2. Globigerina anguliofficinalis Blow, 1969. Plate 11, Figures 8. Globigerina angulisuturalis Bolli, 1957. Plate 12, Figure 3.

Globigerina angustiumbilicata Bolli, 1957. Plate 13, Figure 4. Globigerina bakeri Cole, 1927. Plate 6, Figure 4. Species' concept of Blow (1979) is retained. 
Globigerina binaiensis Koch, 1935. Plate 12, Figures 7-8. The flattened apertural face distinguishes this species from its forerunner G. sellii Borsetti, which is similar in all other respects.

Globigerina ciperoensis Bolli, 1954. Plate 13, Figure 1.

Globigerina corpulenta Subbotina, 1953. Plate 7, Figure 9. The main characteristics of this species are the large and robust tests, the deeply incised sutures of the last whorl, and the relatively high trochospire.

Globigerina daubjergensis Brönnimann, 1953. Plate 1, Figure 4.

Globigerina druryi Akers, 1955. Plate 16, Figure 12.

Globigerina euapertura Jenkins, 1961. Plate 10, Figure 5; Plate 11, Figure 10. (Globigerina prasaepsis Blow, 1969).

Globigerina frontosa Subbotina, 1953. Plate 4, Figure 9.

Globigerina galavisi Bermudez, 1961. Plate 8, Figure 9; Plate 10, Figure 6; Plate 14, Figures 6-7 (G. yeguaensis Weinzierl and Applin, 1929; Blow and Banner, 1962; G. pseudovenezuelana Blow and Banner, 1962).

Globigerina gortanii Borsetti, 1959. Plate 11, Figure 4; Plate 15, Figure 4. The loftiness of its spire distinguishes $G$. gortanii from other species. G. praeturritilina Blow, 1969, could be a subspecific variant of gortanii. We distinguish however, this form on the basis of the height of the spire. The stratigraphic range these two forms were combined. G. gortanii appears in Zone P20.

Globigerina higginsi (Bolli, 1957). Plate 7, Figure 7. No supplementary apertures are observed on the specimens, but the general morphology agrees with the definition of $G$. higginsi.

Globigerina hornibrooki Brönnimann, 1952. Plate 4, Figures 4-5.

Globigerina labiacrassata Jenkins, 1965. Plate 12, Figure 4; Plate 14, Figure 8; Plate 15, Figure 7.

Globigerina linaperta Finlay, 1939. Plate 7, Figure 8; Plate 9, Figure 1.

Globigerina nepenthes Todd, 1957. Plate 17, Figure 12; Plate 18, Figure 2.

Globigerina praebulloides Blow, 1959. Plate 13, Figure 5 (G. praebulloides leroyi Banner and Blow, G. praebulloides occlusa Banner and Blow).

Globigerina praeturritilina Blow and Banner, 1962. Plate 10, Figure 9. See $G$. gortanii.

Globigerina primitiva (Finlay, 1947). Plate 6, Figure 1. See G. triplex. Globigerina pseudoeocaena Subbotina, 1953. Plate 8, Figure 1.

Globigerina sellii Borsetti, 1959. Plate 9, Figure 3; Plate 13, Figure 9. See $G$. binaiensis.

Globigerina senilis Bandy, 1949. Plate 11, Figure 9.

Globigerina spiralis Bolli, 1957. Plate 2, Figure 11.

Globigerina triangularis White, 1928. Plate 2, Figure 10. Concept of Bolli (1957, plate 15, figs. 12-14).

Globigerina triloculinoides Plummer, 1953. Plate 1, Figure 5.

Globigerina tripartita Koch, 1926. Plate 9, Figure 2; Plate 13, Figures $10-11$.

Globigerina triplex (Subbotina, 1953). Plate 6, Figure 2. This species differs from $G$. primitiva (Finlay) in a tighter arrangement of chambers, an narrower aperture, and more or less extraumbilical.

Globigerina velascoensis Cushman, 1925. Plate 2, Figure 9.

Globigerina venezuelana Hedberg, 1937. Plate 15, Figure 1.

Globigerina woodi Jenkins, 1960. Plate 15, Figure 2.

Globigerinatheka barri Brönnimann, 1952. Plate 8, Figure 12. These forms have a typical kugleri morphology, but they possess bullae and have a smaller final chamber.

Globigerinatheka index Finlay, 1939. Plate 9, Figure 8.

Globigerinatheka kugleri (Bolli, Loeblich, and Tappan, 1957). Plate 7, Figure 4. This species is slightly more compact than G. mexicana; distinction between the two is difficult. They are added together in their range (Table 6).

Globigerinatheka luterbacheri Bolli, 1972. Plate 7, Figures 2-3; Plate 8, Figure 11. It is characterized by its elongate test, thick calareous wall, and large size.

Globigerinatheka mexicana (Cushman, 1925). Plate 7, Figure 1.

Globigerinatheka semiinvoluta spp. Plate 8, Figures 7-8. No typical specimens of the Keijzer's species have been observed. The population is like that described by Toumarkine $(1978$, p. 687).

Globigerinatheka senni (Beckmann, 1953). Plate 6, Figure 3.

Globigerinatheka subconglobata (Shutskaya, 1958). Plate 5, Figures 8-9.

Globigerinatheka tropicalis (Blow and Banner, 1962). Plate 8, Figure 10.

Globigerinita africana Blow and Banner, 1962. Plate 10, Figure 3.
Globigerinita globiformis Blow and Banner, 1962. Plate 6, Figure 10. Globigerinita glutinata (Egger, 1893). Plate 16, Figures 9-10. G. incrusta Akers, 1955; Globigerina juvenilis Bolli, 1957; Globigerinita ambitacrena (Loeblich and Tappan, 1957); G. naparimaensis Brönnimann, 1951.

Globigerinita howei Blow and Banner, 1962. Plate 8, Figure 6. Globigerinita martini Blow and Banner, 1962. Plate 6, Figure 9.

Globigerinita pera (Todd, 1957). Plate 7, Figure 11. Globigerinita simulans (Bermudez, 1961). Plate 7, Figure 10.

Globigerinita taroubaensis (Brönnimann, 1952). Plate 4, Figure 8.

Globigerinita turgida (Finlay, 1939). Plate 5, Figure 5.

Globigerinita riveroae Bermudez, 1961. Plate 9, Figure 11; Plate 12, Figure 6.

Globigerinoides altiaperturus Bolli, 1957. Plate 14, Figures 11-12; Plate 15, Figure 6.

Globigerinoides extremus Bolli and Bermudez, 1965. Plate 17, Figure 9.

Globigerinoides primordius Blow and Banner, 1962. Plate 14, Figure 3.

Globigerinoides sacculiferus (Brady, 1887). Plate 17, Figure 10.

Globigerinoides sicanus de Stefani, 1952. Plate 15, Figure 8 (G. bisphaericus Todd).

Globigerinoides seigliei Bermudez and Bolli, 1969. Plate 17, Figures 6-7 (G. tyrrhenicus Borsetti and Cali, 1974).

Globoquadrina altispira (Cushman and Jarvis, 1936). Plate 18, Figure 1.

Globoquadrina baroemoenensis (Le Roy, 1939). Plate 12, Figure 2; Plate 15, Figure 5.

Globoquadrina dehiscens (Chapman, Parr, and Collins, 1934). Plate 16 , Figure 4 ( $G$. dehiscens advena Bermudez, 1949).

Globoquadrina globularis Bermudez, 1961. Plate 12, Figure 1.

Globoquadrina praedehiscens Blow and Banner, 1962. Plate 13, Figure 8.

Globoquadrina? spiny form. Plate 9, Figures 4-7, 10. (Globoquadrina sp., Boersma, 1977, plate 2, figs. 8-10). Several specimens are recognized in the lower Miocene. The general outline of these forms corresponds to the genus Globoquadrina?, but they possess a bulla or a little chamber on the umbilicus. Moreover, these forms are spiny; the spines are more or less elongate.

Globorotalia acarinata (Subbotina, 1953). Plate 3, Figure 6.

Globorotalia aequa Cushman and Renz, 1942. Plate 3, Figure 12.

Globorotalia angulata (White, 1928). Plate 2, Figure 7.

Globorotalia angulosa (Bolli, 1957). Plate 3, Figure 11. The specimens observed have $31 / 2$ (rarely 4 ) chambers in the last whorl. The umbilical shoulders are distinctly angular. The first whorl is slightly prominent.

Globorotalia aragonensis Nuttal, 1930. Plate 4, Figure 2.

Globorotalia aspensis (Colom, 1954). Plate 4, Figures 14-15.

Globorotalia broedermanni Cushman and Bermudez, 1949. Plate 4, Figures 10-11; Plate 5, Figure 6; Plate 6, Figure 5. Different aspects of the population are illustrated. The specimens of Plate 4 (Figures 10-11) are the more primitive in the sequence (probably Zone P10). But they have a biconvex test, 5-6 chambers in the last whorl, and a slightly lobate peripheral margin. However, the apertural face is not so prominent as for the specimens observed in Zones P11-P13 (Plate 5, Figure 6), and the walls of the chambers are more coarsely perforated.

Globorotalia bullbrooki Bolli, 1957. Plate 5, Figures 1-2; Plate 6, Figure 6. Some specimens often associated with typical G. bullbrooki are regarded as $G$. cf. bullbrooki (Plate 6, Figure 6). They have no deep sutures on the ventral side, but have a tighter arrangement of the chambers of last whorl; the last chambers are slightly above the umbilicus.

Globorotalia cerroazulensis (Cole, 1928). Plate 10, Figure 1.

Globorotalia chapmani Parr, 1938. Plate 3, Figure 3.

Globorotalia cibaoensis Bermudez, 1949. Plate 17, Figure 13.

Globorotalia cocoaensis Cushman, 1928. Plate 10, Figure 2.

Globorotalia collactea Finlay, 1933. Plate 6, Figure 8.

Globorotalia compressa (Plummer, 1953). Plate 2, Figures 2-4.

Globorotalia conicotruncata (Subbotina, 1947). Plate 2, Figure 8.

Globorotalia conoidea Walters, 1965. Plate 17, Figures 4-5.

Globorotalia conomiozea Kennett, 1966. Plate 18, Figures 3-6.

Globorotalia continuosa Blow, 1959. Plate 11, Figure 3; Plate 13, Figure 7; Plate 15, Figure 3. The concept of $G$. pseudocontinuosa Jenkins (1967) is included in the stratigraphic range of this taxon. The illustration gives an idea of the variability of the population accepted here. 
Globorotalia convexa Subbotina, 1953. Plate 3, Figures 1-2.

Globorotalia crassaformis Galloway and Wissler, 1927. Plate 18, Figures 7,13 . We agree with the lineage $G$. puncticulata-G. crassaformis described by Berggren (1977b). The figured specimen is typical of the earlier stage of the evolution.

Globorotalia crassula Cushman and Stewart, 1930. Plate 18, Figures 10-12; Plate 19, Figures 1,12. Species' concept of Berggren and Amdurer (1973) and Berggren (1977b).

Globorotalia crater Finlay, 1939. Plate 4, Figure 1.

Globorotalia exilis Blow, 1969. Plate 19, Figure 10. See G. pertenuis Beard.

Globorotalia haunsbergensis Gohrbandt, 1963. Plate 2, Figures 5-6. The spiral side is more convex than in G. ehrenbergi Bolli, and the septal sutures are more curved.

Globorotalia inconstans (Subbotina, 1953). Plate 1, Figure 7.

Globorotalia inflata (d'Orbigny, 1839). Plate 19, Figure 2.

Globorotalia intermedia (Subbotina, 1953). Plate 3, Figure 7. These forms are characterized by a tighter arrangement of chambers of the last whorl and a rounded outline of the test.

Globorotalia kugleri Bolli, 1957. Plate 14, Figures 1-2. In the stratigraphic range, G. pseudokugleri Blow (1969) is added (Plate 14, Figure 2).

Globorotalia lehneri Cushman and Jarvis, 1929. Plate 5, Figure 4.

Globorotalia lenguaensis Bolli, 1957. Plate 17, Figures 2-3.

Globorotalia lensiformis Subbotina, 1953. Plate 3, Figure 14.

Globorotalia margaritae Bolli and Bermudez, 1965. Plate 18, Figures 8-9 (G. margaritae evoluta Cita, 1973).

Globorotalia mckannai (White, 1928). Plate 3, Figure 5.

Globorotalia cf. medizzai (Toumarkine and Bolli, 1975). Plate 8, Figures $4-5$. Few specimens with only 4 to $41 / 2$ chambers in the last whorl are recognized. They can be integrated in the variation of the species population described by the authors.

Globorotalia menardii (Parker, Jones, and Brady, 1865). ex d'Orbigny. Plate 17, Figures 1 . (G. cultrata d'Orbigny, 1839).

Globorotalia mendacis Blow, 1969. Plate 12, Figures 9-10; Plate 13, Figure 6; Plate 14, Figure 4.

Globorotalia miocenica Palmer, 1945. Plate 19, Figures 3, 7-8, 11, 13. Some specimens are large multicamerate with a slight biconvexity (Plate 19, Figure 3). They appear sooner in the Pliocene sequence than the specimens with a flat spiral side.

Globorotalia miozea Finlay, 1939. Plate 16, Figure 1.

Globorotalia multicamerata Cushman and Jarvis, 1930. Plate 19, Figure 5.

Globorotalia obesa Bolli, 1957. Plate 11, Figure 5.

Globorotalia occlusa Loeblich and Tappan, 1957. Plate 3, Figure 8.

Globorotalia opima nana Bolli, 1957. Plate 11, Figure 6.

Globorotalia opima opima Bolli, 1957. Plate 11, Figure 7.

Globorotalia pentacamerata Subbotina, 1947. Plate 5, Figure 3.

Globorotalia peripheroronda Blow and Banner, 1966. Plate 16, Figure 8.

Globorotalia pertenuis Beard, 1969. Plate 19, Figures 4, 6, 9. This species differs from $G$. exilis Blow in its more numerous and lobate chambers. These two species have the same stratigraphic distribution at the four sites of Leg 72 .

Globorotalia pomeroli Toumarkine and Bolli, 1970. Plate 8, Figure 3.

Globorotalia possagnoensis Toumarkine and Bolli, 1970. Plate 8, Figure 2.

Globorotalia praecursoria (Morozova, 1957). Plate 1, Figures 8-10. Globorotalia trinidadensis Bolli (1957) is considered a junior synonym.

Globorotalia praemenardii Cushman and Stainforth, 1945. Plate 16, Figures 2-3.

Globorotalia prolata (Bolli, 1957). Plate 4, Figures 12-13. The concept of Blow (1979, p. 856) is retained.

Globorotalia pseudobulloides (Plummer, 1953). Plate 1, Figure 6.

Globorotalia pseudomenardii Bolli, 1957. Plate 3, Figure 4.

Globorotalia puncticulata (Deshayes, 1832). Plate 18, Figure 14.

Globorotalia pusilla Bolli, 1957. Plate 2, Figures 12-13. It corresponds to the $G$. pusilla pusilla form of the author.

Globorotalia siakensis Le Roy, 1939. Plate 11, Figure 1; Plate 14, Figure 5. Some specimens referable to $G$. semivera Hornibrook (1961) are grouped with this species.

Globorotalia scitula (Brady, 1882). Plate 16, Figure 6; Plate 17, Figure 8 . Some specimens referable to $G$. praescitula Blow (1959) are recognized at the top of Zone N6. They are not separated according to stratigraphic range.

Globorotalia soldadoensis (Brönnimann, 1952). Plate 3, Figure 10. Globorotalia sphericonomiozea Walters, 1965. Plate 17, Figure 11.

Globorotalia spinulosa Cushman, 1927. Plate 5, Figure 7; Plate 7, Figure 6.

Globorotalia subbotinae Morozova, 1939. Plate 3, Figure 13.

Globorotalia uncinata Bolli, 1957. Plate 1, Figure 11; Plate 2, Figure 1. Globorotalia velascoensis (Cushman, 1925). Plate 3, Figure 9.

Globorotalia zealandica Hornibrook, 1958. Plate 15, Figures 9-12. The different "subspecies" of Walters (1965) (G. zealandica incognita, G. zealandica pseudomiozea) are not separated, partly because of their poor representation.

Globorotaloides suteri Bolli, 1957. Plate 12, Figure 11.

Globorotaloides testarugosa (Jenkins, 1960). Plate 13, Figures 2-3.

Hantkenina sp. Plate 6, Figure 7. Only fragments of specimens are observed. No specific determination is made.

Praeorbulina sp. Plate 16, Figure 7. The different subspecies of glomerosa Blow (1956) are not differentiated.

Praeorbulina transitoria (Blow, 1956). Plate 16, Figure 5.

Pseudohastigerina barbadoensis Blow, 1969. Plate 9, Figure 9.

Pseudohastigerina micra (Cole, 1927). Plate 4, Figure 7.

Pseudohastigerina wilcoxensis (Cushman and Ponton, 1932). Plate 4, Figure 6.

Sphaeroidinellopsis seminulina (Schawger, 1866). Plate 16, Figure 11. Truncorotaloides topilensis (Cushman, 1925). Plate 5, Figures 10-11; Plate 7, Figure 5.

\section{ACKNOWLEDGMENTS}

I wish to express my sincere thanks to the authorities of the Deep Sea Drilling Project for their kind invitation to participate onboard the Glomar Challenger for Leg 72.

I should like to thank M. Le Blanc of the Centre de Microscopie Electronique of the Université de Bordeaux. The Institut de Géologie du Bassin d'Aquitaine provided laboratory facilities and technical help. I thank W. Berggren and D. Johnson (Woods Hole Oceanographic Institution) and W. Coulbourn (Scripps Institution of Oceanography) for their helpful comments on this manuscript. I wish to thank my coscientists on Leg 72 and particularly L. Tjalsma and P. Cepek for valuable discussion on Leg 72 biostratigraphy problems.

\section{REFERENCES}

Berggren, W. A., 1971. Multiple phylogenetic zonations of the Cenozoic based on planktonic Foraminifera. In Farinacci, A. (Ed.), Proc. II Plankt. Conf. Roma: Rome (Edizioni Tecnoscienza), $1: 41-56$

1973. The Pliocene time scale: calibration of planktonic foraminiferal and calcareous nannoplankton zones. Nature, 243(5407):391-397.

1977a. Late Neogene planktonic foraminiferal biostratigraphy of DSDP Site 357 (Rio Grande Rise). In Supko, P. R., PerchNielsen, K., et al., Init. Repts. DSDP, 39: Washington (U.S. Govt. Printing Office), 591-614.

1977b. Late Neogene planktonic foraminiferal biostratigraphy of the Rio Grande Rise (South Atlantic). Mar. Micropaleontol., 2:265-313.

Berggren, W. A., and Amdurer, M., 1973. Late Paleogene (Oligocene) and Neogene planktonic foraminiferal biostratigraphy of the Atlantic Ocean (Lat. $3^{\circ} \mathrm{N}$ to Lat. $30^{\circ} \mathrm{S}$ ). Riv. Ital. Paleontol., 79: 337-392.

Blow, W. H., 1969. Late middle Eocene to Recent planktonic foraminiferal biostratigraphy. In Brönniman, P., and Renz, H. H. (Eds.), Proc. First. Int. Conf. Plankt. Microfossils: Leiden (E. J. Brill), 1:199-422.

1979. The Cainozoic Globigerinida: Leiden (E. J. Brill).

Boersma, A., 1977. Cenozoic planktonic Foraminifera, DSDP Leg 39 (South Atlantic). In Supko, P. R., Perch-Nielsen K., et al., Init. Repts. DSDP, 39: Washington (U.S. Govt. Printing Office), 567-590.

Bolli, H. M., 1957. The genera Globigerina and Globorotalia in the Paleocene-lower Eocene Lizard Springs Formation of Trinidad, B. W. I. U.S. Nat. Mus. Bull., 215:155-172. 
1966. Zonation of Cretaceous to Pliocene marine sediments based on planktonic Foraminifera. Bol. Inf. Asoc. Venez. Geol. Min. Pet., 9:3-32.

Jenkins, D. G., 1960. Planktonic Foraminifera from the Lakes Entrace oil shaft, Victoria, Australia. Micropaleontology, 6:345-371.

1971. Cenozoic planktonic Foraminifera of New Zealand. N. Z. Geol. Surv. Paleontol. Bull., 42:1-278.

1975. Cenozoic planktonic foraminiferal biostratigraphy of the southwestern Pacific and Tasman Sea, DSDP Leg 29. In Kennett, J. P., Houtz, R. E., et al., Init. Repts. DSDP, 29: Washington (U.S. Govt. Printing Office), 449-467.

1978. Neogene planktonic foraminifers from DSDP Leg 40, Sites 360 and 362 in the southeastern Atlantic. In Bolli, H. M., Ryan, W. B. F., et al., Init. Repts. DSDP, 40: Washington (U.S. Govt. Printing Office), 723-739.
Maxwell, A. E., Von Herzen, R. P., et al., 1970. Init. Repts. DSDP, 3: Washington (U.S. Govt. Printing Office).

Stainforth, R. M., Lamb, J. L., Luterbacher, H., Beard, J. H., and Jeffords, R. M., 1975. Cenozoic planktonic foraminiferal zonation and characteristics of index forms. Contrib. Univ. Kans., Article 62 .

Supko, P. R., Perch-Nielsen, K., et al., 1977. Init. Repts. DSDP, 39: Washington (U.S. Govt. Printing Office).

Toumarkine, M., 1978. Planktonic foraminiferal biostratigraphy of the Paleogene of Site 360 to 364 and the Neogene of Sites 362A, 363, and 364, Leg 40. In Bolli, H. M., Ryan, W. B. F. et al., Init. Repts. DSDP, 40: Washington (U.S. Govt. Printing Office), 679-722.

Date of Initial Receipt: May 6, 1982 
APPENDIX

List of Samples without Planktonic Foraminifers, Holes 515A and 515B (core-section, interval in $\mathrm{cm}$ )

\begin{tabular}{|c|c|c|c|c|}
\hline Hole $515 \mathrm{~A}$ & Hole 515B & Hole 515B (Cont.) & Hole 515B (Cont.) & Hole 515B (Cont.) \\
\hline $1-3,90-93$ & $1-1,60-62$ & $14-4,30-32$ & $30-3,50-52$ & $42, \mathrm{CC}$ \\
\hline $1, \mathrm{CC}$ & $1-2,60-62$ & $14-5,30-32$ & $30-4,50-52$ & $43-1,51-53$ \\
\hline $2-1,84-86$ & $1-3,60-62$ & $14-6,30-32$ & $30-5,50-52$ & $43-2,94-97$ \\
\hline $2-2,36-38$ & $1-4,60-62$ & $14, \mathrm{CC}$ & $30-6,50-52$ & $43-3,63-65$ \\
\hline $2-2,47-49$ & $1, \mathrm{CC}$ & $15-1,30-32$ & $30-7,50-52$ & $43-4,43-44$ \\
\hline $2-3,44-46$ & $2-1,122-124$ & $15-2,30-32$ & $30, \mathrm{CC}$ & $43-5,63-65$ \\
\hline $2, \mathrm{CC}$ & $2-2,135-137$ & $15-3,30-32$ & $31-1,50-52$ & $43-6,25-27$ \\
\hline $3-1,54-56$ & $2, \mathrm{CC}$ & $15-4,30-32$ & $31-2,50-52$ & $43, \mathrm{CC}$ \\
\hline $3-2,54-56$ & $3-1,60-62$ & $15-5,34-36$ & $31-3,50-52$ & $44-1,76-79$ \\
\hline $3-3,54-56$ & $3-2,60-62$ & $15, \mathrm{CC}$ & $31-4,40-46$ & $44-2,70-72$ \\
\hline $4-2,83-85$ & $3-3,50-62$ & $16-1,30-32$ & $31-5,50-52$ & $44-3,52-54$ \\
\hline $4-3,83-85$ & $3-4,65-67$ & $16-2,30-32$ & $31-6,50-52$ & $44-4,37-40$ \\
\hline $4, \mathrm{CC}$ & $3-6,100-102$ & $16-3,30-32$ & $31-7,50-52$ & $44-5,20-23$ \\
\hline $5-1,55-57$ & $3, \mathrm{CC}$ & $16-4,30-32$ & $31, \mathrm{CC}$ & $44-6,57-59$ \\
\hline $5-2,55-57$ & $4-1,95-97$ & $16-5,30-32$ & $32-1,115-117$ & $44, C C$ \\
\hline $5-3,55-57$ & $4-2,95-97$ & $16, \mathrm{CC}$ & $32-2,115-117$ & $45-1,8-9$ \\
\hline $6-1,103-105$ & $4-3,95-97$ & $17-1,86-89$ & $32-3,62-64$ & $45-1,10-12$ \\
\hline $6-2,103-105$ & $4-4,95-97$ & $17-2,86-89$ & $32-4,115-117$ & $45-2,130-131$ \\
\hline $7-3,103-105$ & $4-5,95-97$ & $17-3,86-89$ & $32-5,110-117$ & $45-3,91-92$ \\
\hline $7, \mathrm{CC}$ & $4-6,95-97$ & $17, \mathrm{CC}$ & $32-6,96-98$ & $45-4,130-131$ \\
\hline $8-1,104-106$ & $5-2,95-97$ & $18-1,40-42$ & $32-7,42-46$ & $45-5,132-134$ \\
\hline $8-2,104-106$ & $5-3,95-97$ & $18-2,40-42$ & $32, \mathrm{CC}$ & $45-6,43-45$ \\
\hline $8, \mathrm{CC}$ & $5-4,95-97$ & $18, \mathrm{CC}$ & $33-1,93-95$ & $45, \mathrm{CC}$ \\
\hline $9-1,103-105$ & $5, \mathrm{CC}$ & $19-1,90-92$ & $33-2,94-96$ & $46-1,103-104$ \\
\hline $9-2,103-105$ & $6-1,60-64$ & $19-2,90-92$ & $33-3,96-98$ & $46-1,145-147$ \\
\hline $9-3,103-105$ & $6-1,95-97$ & $19-3,90-92$ & $33-4,113-115$ & $46-2,71-73$ \\
\hline $10-1,103-105$ & $6-2,95-97$ & $19-3,90-92$ & $33-5,101-103$ & $46-2,103-105$ \\
\hline $10-3,101-103$ & $6-2,135-139$ & $19-4,90-92$ & $33-6,71-73$ & $46-3,103-105$ \\
\hline $10, \mathrm{CC}$ & $6-3,60-64$ & $19, \mathrm{CC}$ & $33-7,5-7$ & $46-4,88-90$ \\
\hline $11-1,103-105$ & $6-3,95-97$ & $20-1,120-122$ & $33, \mathrm{CC}$ & $46-5,94-96$ \\
\hline $11-2,103-105$ & $6-4,95-97$ & $20-2,120-122$ & $34-1,115-117$ & $46-6,97-98$ \\
\hline $11-3,100-101$ & $6-4,137-141$ & $20-3,120-122$ & $34-2,122-124$ & $46-7,60-61$ \\
\hline $11, \mathrm{CC}$ & $6-5,43-47$ & $20-4,120-122$ & $34-3,119-121$ & $46, C C$ \\
\hline $12-1,0-2$ & $6-5,60-64$ & $20-5,120-122$ & $34-4,127-129$ & $47-1,90-91$ \\
\hline $12-1,103-105$ & $6-5,70-74$ & $20-6,100-102$ & $34-5,105-107$ & $47-2,91-93$ \\
\hline $12-2,103-105$ & $6-5,95-97$ & $20, \mathrm{CC}$ & $34-6,107-109$ & $47-4,114-116$ \\
\hline $12-3,95-97$ & $6-6,111-115$ & $21-1,95-96$ & $34-7,42-44$ & $47-5,88-90$ \\
\hline $12, \mathrm{CC}$ & $6-7,5-9$ & $21-2,95-96$ & $34, \mathrm{CC}$ & $47, \mathrm{CC}$ \\
\hline $13-1,103-105$ & $7-1,22-23$ & $21-3,95-96$ & $35-1,124-126$ & $48-1,88-90$ \\
\hline $13-2,103-105$ & $7-2,22-23$ & $21-4,95-96$ & $35-2,125-127$ & $48-2,88-90$ \\
\hline $13-3,90-92$ & $7-3,22-23$ & $21-5,95-96$ & $35-3,125-127$ & $48-3,88-90$ \\
\hline $13, \mathrm{CC}$ & $7, \mathrm{CC}$ & $21-6,96-96$ & $35-4,102-104$ & $48-4,88-90$ \\
\hline $14-1,54-56$ & $8-1,85-87$ & $21, \mathrm{CC}$ & $35-5,109-111$ & $49-5,46-49$ \\
\hline $14-2,54-56$ & $8-2,56-60$ & $22-1,96-98$ & $35-6,65-67$ & $49, \mathrm{CC}$ \\
\hline $14-3,54-56$ & $8-2,85-87$ & $22-2,96-98$ & $35-7,36-38$ & $50-1,0-3$ \\
\hline $14, \mathrm{CC}$ & $8-3,85-87$ & $22-3,96-98$ & $36-1,7-10$ & $50-3,141-143$ \\
\hline $16-1,20-22$ & $8-4,56-60$ & $22-4,96-98$ & $36-2,43-45$ & $50-4,85-91$ \\
\hline $16-2,20-22$ & $8-4,85-87$ & $22-5,96-98$ & $36-3,8-12$ & $50-5,105-109$ \\
\hline $16-3,20-22$ & $8-4,130-132$ & $23-1,109-110$ & $36-4,62-64$ & $50-6,110-112$ \\
\hline $16, \mathrm{CC}$ & $8, \mathrm{CC}$ & $23-2,109-110$ & $36-5,42-45$ & $50, \mathrm{CC}$ \\
\hline $17-1,20-22$ & $9, \mathrm{CC}$ & $23-3,109-110$ & $36-6,24-26$ & $51-1,23-25$ \\
\hline $17-2,20-22$ & $10-1,30-32$ & $23-5,109-110$ & $36, \mathrm{CC}$ & $51-2,104-106$ \\
\hline $17-3,20-22$ & $10-3,30-32$ & $25-1,55-56$ & $37-1,78-80$ & $51, \mathrm{CC}$ \\
\hline $17, \mathrm{CC}$ & $10, \mathrm{CC}$ & $25-3,55-56$ & $37-2,37-38$ & $52, \mathrm{CC}$ \\
\hline $18-1,20-22$ & $11-1,30-32$ & $25-5,55-56$ & $37-4,105-106$ & $53-1,59-61$ \\
\hline $18-2,20-22$ & $11-2,30-32$ & $25, \mathrm{CC}$ & $37, \mathrm{CC}$ & $53-2,48-50$ \\
\hline $18-3,20-22$ & $11-3,30-32$ & $26-1,95-97$ & $38-1,41-42$ & $53-3,26-28$ \\
\hline $18, \mathrm{CC}$ & $11-4,30-32$ & $26-2,10-12$ & $38-2,75-78$ & $53-5,43-45$ \\
\hline $19-1,70-72$ & $11-5,30-32$ & $26, \mathrm{CC}$ & $38-3,37-40$ & $53-6,71-73$ \\
\hline $19, \mathrm{CC}$ & $11-6,30-32$ & $27-1,147-148$ & $38-4,31-34$ & $54-1,53-55$ \\
\hline $21-1,20-22$ & $11, \mathrm{CC}$ & $27-3,147-148$ & $38-5,84-85$ & $55-1,70-72$ \\
\hline $21-2,20-22$ & $12-1,40-42$ & $27-4,147-148$ & $38-6,60-63$ & $55-1,106-108$ \\
\hline $21, \mathrm{CC}$ & $12-2,40-42$ & $27-5,147-148$ & $38, \mathrm{CC}$ & $55-3,75-77$ \\
\hline $22-1,20-22$ & $12-2,110-113$ & $27-6,140-142$ & $39-1,20-21$ & $55-3,131-135$ \\
\hline $22, \mathrm{CC}$ & $12-3,40-42$ & $27-6,147-148$ & $39-2,64-67$ & $55-4,40-42$ \\
\hline $23-1,85-87$ & $12-4,40-42$ & $28-1,131-133$ & $39-3,16-17$ & $55-5,65-67$ \\
\hline $23-2,20-22$ & $12, \mathrm{CC}$ & $28-2,50-52$ & $39-4,64-67$ & $55-6,80-82$ \\
\hline $23-3,20-22$ & $13-1,91-93$ & $28-3,50-52$ & $39-5,11-13$ & $55-6,106-109$ \\
\hline $23, \mathrm{CC}$ & $13-2,91-93$ & $28-4,50-52$ & $39, \mathrm{CC}$ & $55, \mathrm{CC}$ \\
\hline $24-1,55-57$ & $13-3,91-93$ & $28-5,50-52$ & $40-1,0-4$ & $56-1,96-103$ \\
\hline $24-2,55-57$ & $13-4,91-93$ & $28-6,50-52$ & $40-2,8-10$ & $56-2,119-124$ \\
\hline $24-3,35-37$ & $13-4,94-98$ & $28-7,50-52$ & $40-3,33-36$ & $56-3,143-150$ \\
\hline $24, \mathrm{CC}$ & $13-5,30-32$ & $28, \mathrm{CC}$ & $40-4,57-59$ & \\
\hline $25-1,75-77$ & $13-5,61-62$ & $29-1,83-84$ & $40-5,26-29$ & \\
\hline $25-2,75-77$ & $13-5,91-93$ & $29-2,83-84$ & $40, \mathrm{CC}$ & \\
\hline $25-3,27-29$ & $13-5,127-128$ & $29-3,83-84$ & $41-1,72-75$ & \\
\hline $25, \mathrm{CC}$ & $13-6,91-93$ & $29-4,83-84$ & $41-2,26-29$ & \\
\hline $26-3,35-37$ & $13, \mathrm{CC}$ & $29-5,83-84$ & $41-3,60-62$ & \\
\hline $26, \mathrm{CC}$ & $14-1,30-32$ & $29-6,83-84$ & $41-4,47-48$ & \\
\hline $27-1,80-82$ & $14-2,30-32$ & $29, \mathrm{CC}$ & $41-5,12-14$ & \\
\hline $27-2,80-82$ & $14-2,40-43$ & $30-1,50-52$ & $41-6,15-17$ & \\
\hline $27, \mathrm{CC}$ & $14-3,30-32$ & $30-2,50-52$ & $41, \mathrm{CC}$ & \\
\hline
\end{tabular}



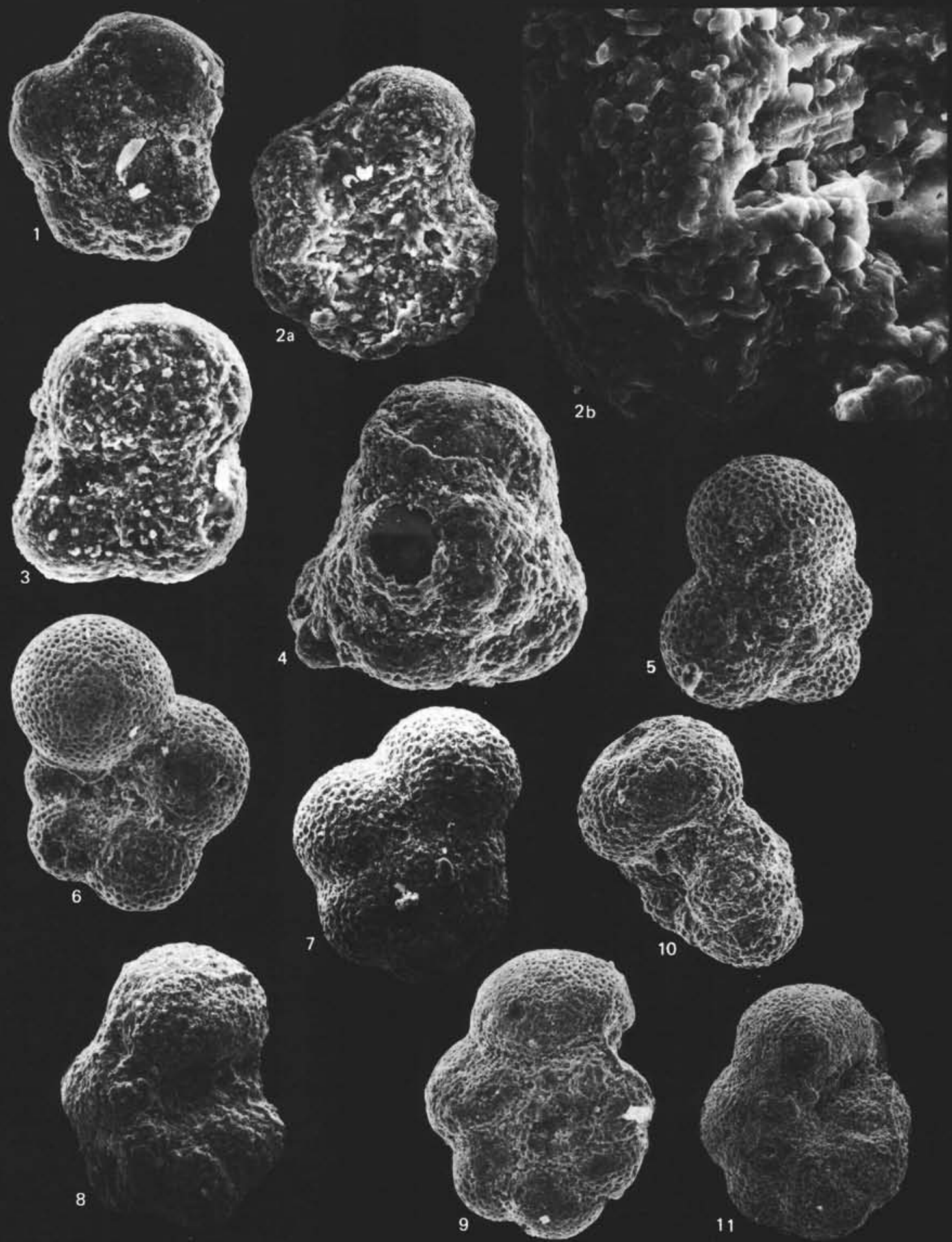

8

10
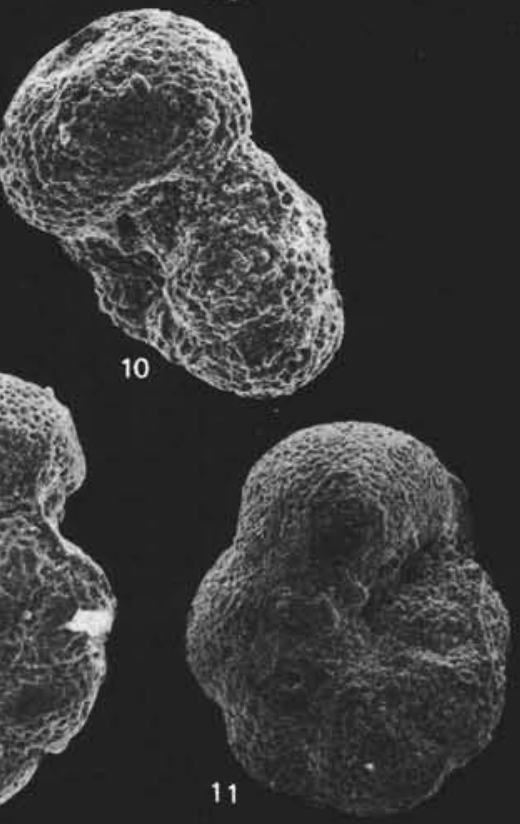

Plate 1. 1-3. Eoglobigerina sp., Sample 516F-89-5, 24-26 cm, (1) spiral view, $\times 230$; (2) (a) umbilical view, $\times 430$, (b) detail, $\times 1720$; (3) umbilical view, $\times 345$. 4. Globigerina daubjergensis Brönnimann, Sample $516 \mathrm{~F}-89-5,14-21 \mathrm{~cm}$, spiral view, $\times 260$. 5-6. Sample $516 \mathrm{~F}-89-2$, $119-121 \mathrm{~cm}$, (5) Globigerina triloculinoides Plummer, spiral view, $\times 155,(6)$ Globorotalia pseudobulloides (Plummer), umbilical view, $\times 155$. 7. Globorotalia inconstans (Subbotina), Sample 516F-88-3, 55-57 cm, spiral view, $\times 120$. 8-10. Globorotalia praecursoria (Morozova), (8) Sample 516F-88-3, $55-57 \mathrm{~cm}$, umbilical view, $\times 120$, (9) Sample $516 \mathrm{~F}-89-2,119-121 \mathrm{~cm}$, spiral view, $\times 150,(10)$ Sample $516 \mathrm{~F}-87-7,39-40 \mathrm{~cm}$, side view, $\times 140$. 11 . Globorotalia uncinata Bolli, Sample 516F-87-7, 39-40 cm, umbilical view, $\times 85$. 

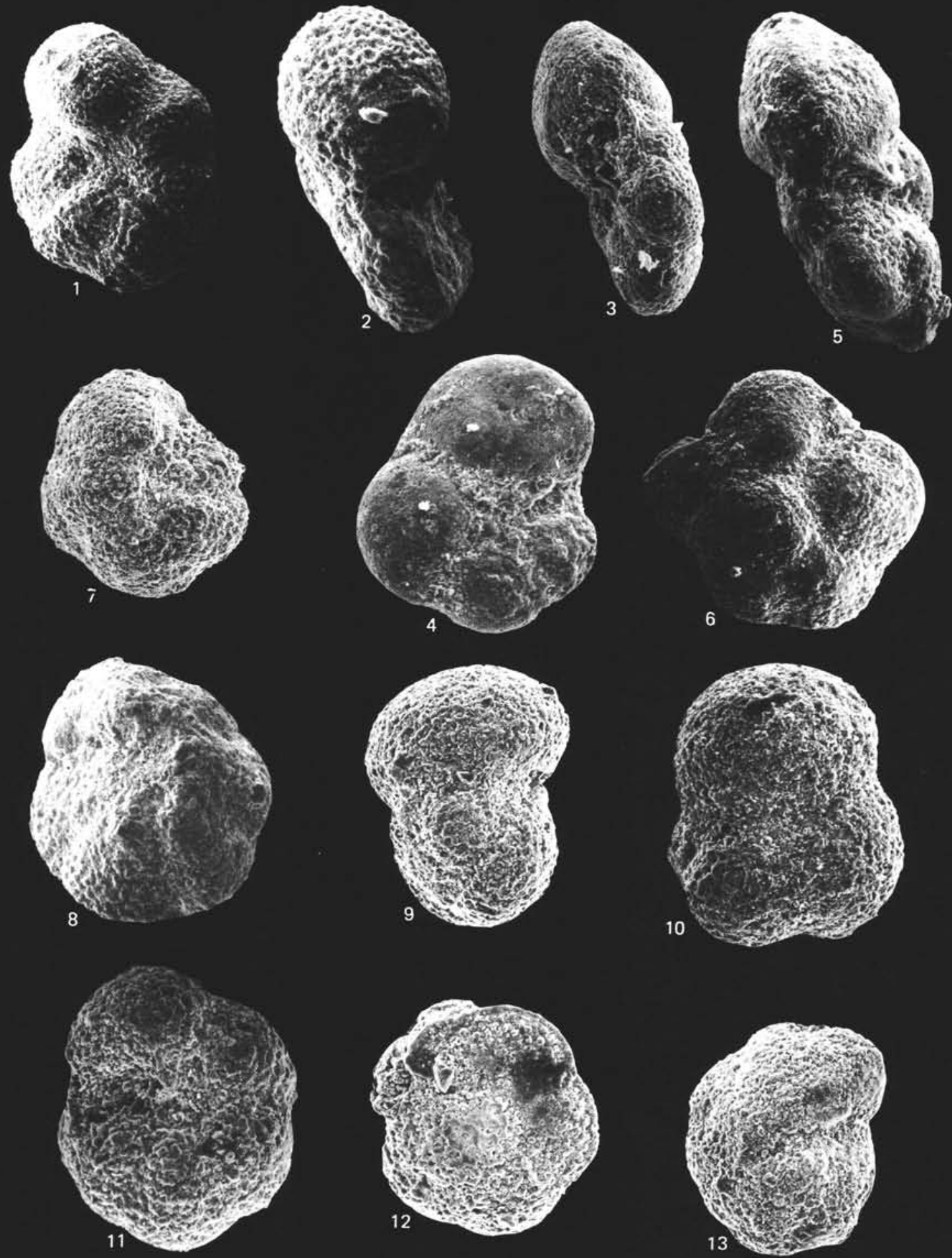

Plate 2. 1. Globorotalia uncinata Bolli, Sample $516 \mathrm{~F}-87-7,39-40 \mathrm{~cm}$, umbilical view, $\times 120$. 2-4. Globorotalia compressa (Plummer), (2) Sample 516F-89-2, 119-121 cm, side view, $\times 200$, (3-4) Sample 516F-88-3, 55-57 cm (3, side view, $\times 150 ; 4$, umbilical view, $\times 140)$. 5-6. Globorotalia haunsbergensis Gohrbandt, Sample 516F-87-5, 48-49 cm, (5) side view, $\times 170$, (6) spiral view, $\times 150$. 7. Globorotalia angulata (White), Sample 516F-88-2, 102-103 cm, umbilical view, $\times 115$. 8. Globorotalia conicotruncata Subbotina, Sample $516 \mathrm{~F}-87-4,43-44 \mathrm{~cm}$, umbilical view, $\times 130$. 9. Globigerina velascoensis Cushman, Sample $516 \mathrm{~F}-86-4,6-9 \mathrm{~cm}$, side view, $\times 120$. 10. Globigerina triangularis White, Sample $516 \mathrm{~F}-85-1,40-42 \mathrm{~cm}$, umbilical view, $\times 130$. 11. Globigerina spiralis Bolli, Sample $516 \mathrm{~F}-87-5,39-40 \mathrm{~cm}$, umbilical view, $\times 180$. 12-13. Globorotalia pusilla Bolli, Sample 516F-86-4, 6-9 cm, (12) spiral view, $\times 130$, (13) umbilical view, $\times 105$. 

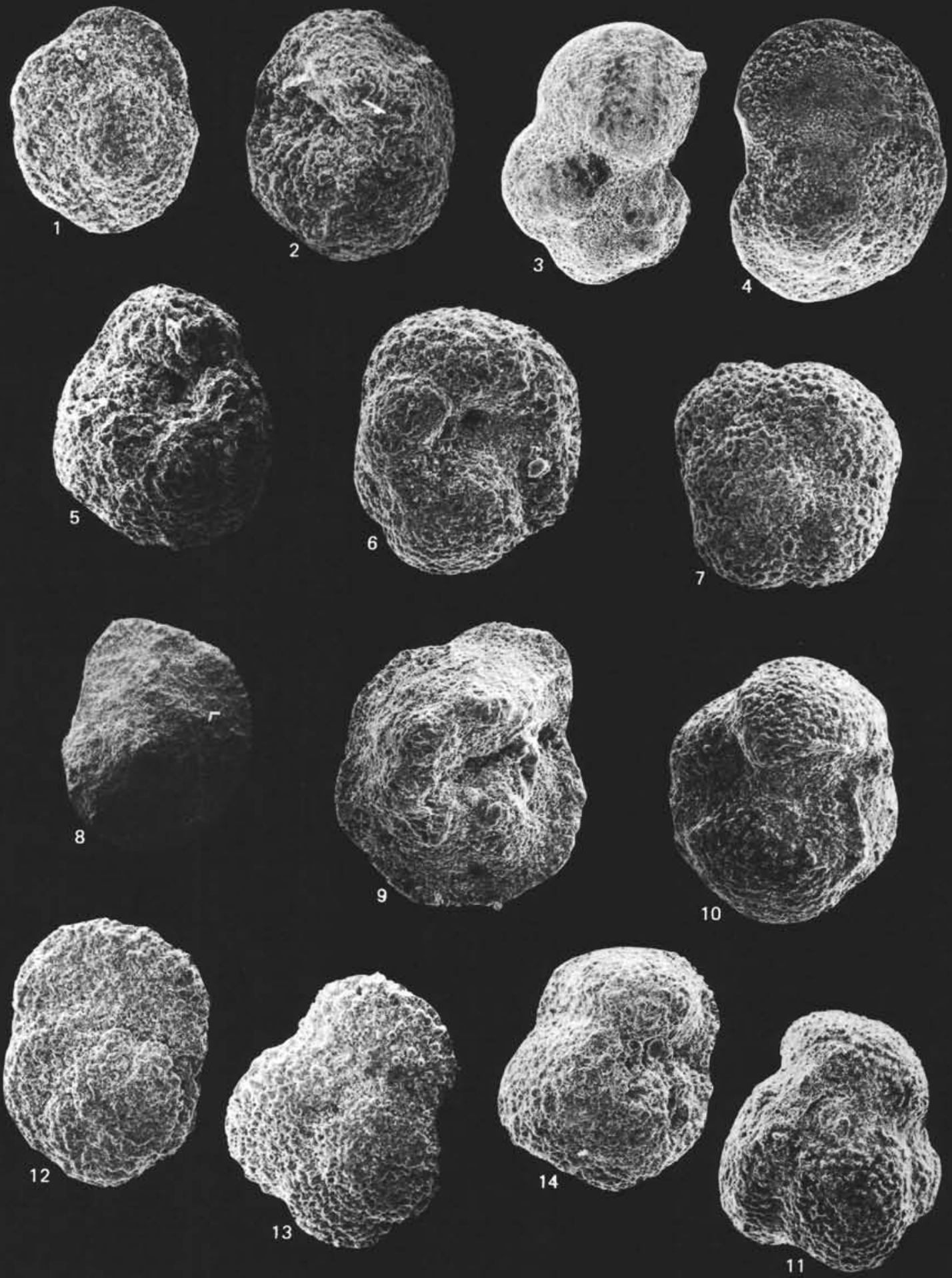

Plate 3. 1-2. Globorotalia convexa Subbotina, Sample 516F-88-2, 102-103 cm, (1) umbilical view, $\times 130,(2)$ spiral view, $\times 140$. 3. Globorotalia chapmani Parr, Sample 516F-85-1, 40-42 cm, spiral view, $\times 80$. 4, 9. Sample 516F-84-5, 13-15 cm, (4) Globorotalia pseudomenardii Bolli, spiral view, $\times 150$, (9) Globorotalia velascoensis (Cushman), umbilical view, $\times 90 . \quad 5,7,12$. Sample 516F-83-5, 22-24 cm, (5) Globorotalia mckannai (White), spiral view, $\times 140$, (7) Globorotalia intermedia (Subbotina), spiral view, $\times 120,(12)$ Globorotalia aequa Cushman and Renzi, spiral view, $\times 105$. 6. Globorotalia acarinata (Subbotina), Sample 516F-85-1, $40-42 \mathrm{~cm}$, umbilical view, $\times 105$. 8. Globorotalia occlusa Loeblich and Tappan, Sample 516F-84-3, 21-25 cm, spiral view, $\times 90.10-11$. Sample $516 \mathrm{~F}-78-4,78-80 \mathrm{~cm}$, (10) Globorotalia soldadoensis (Brönnimann), umbilical view, $\times 90$, (11) Globorotalia angulosa (Bolli), umbilical view, $\times 80 . \quad 13$. Globorotalia subbotinae Morozova, Sample $516 \mathrm{~F}-83-8,22-24 \mathrm{~cm}$, spiral view, $\times 110$. 14. Globorotalia lensiformis Subbotina, Sample $516 \mathrm{~F}-82, \mathrm{CC}$, umbilical view, $\times 70$. 

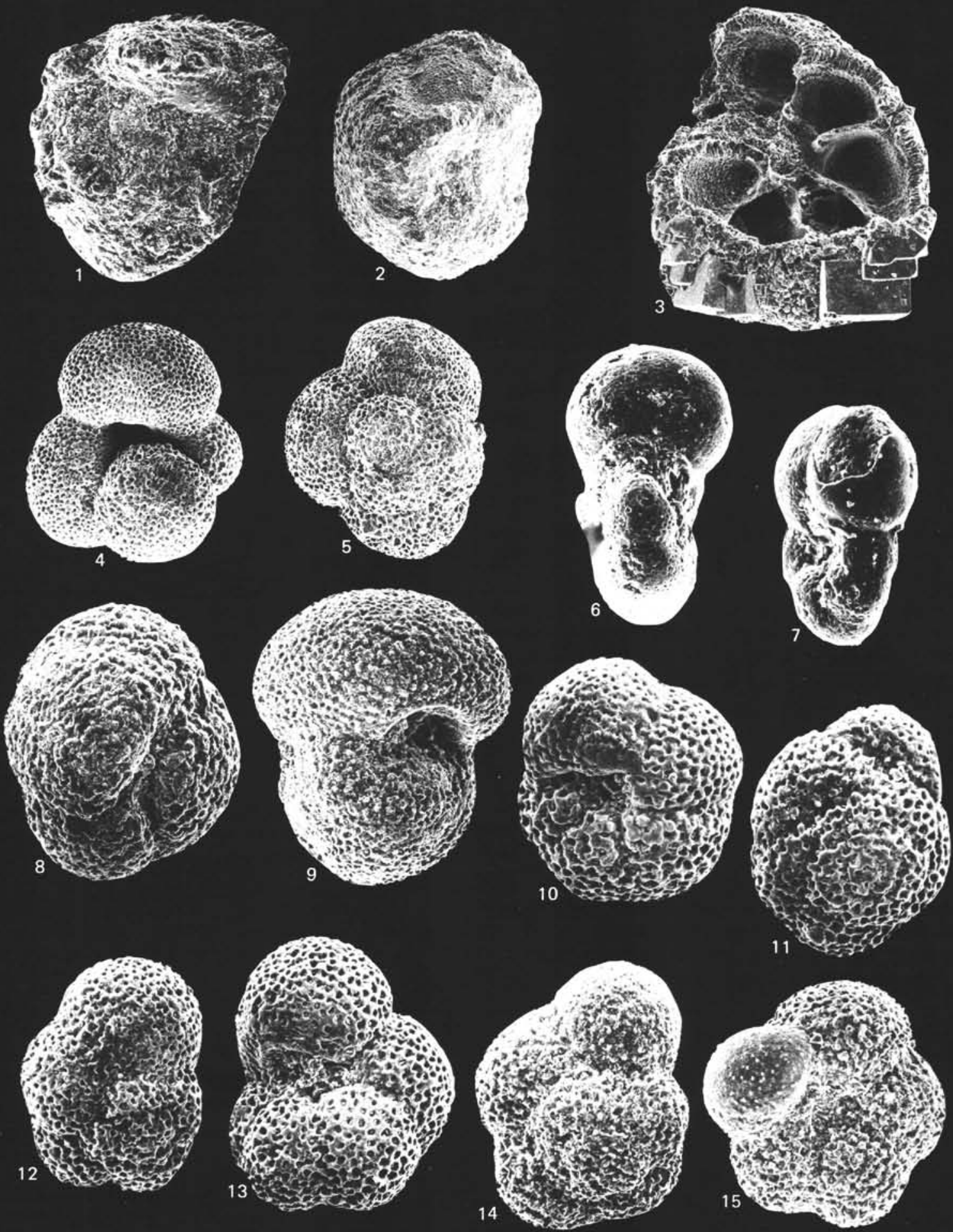

Plate 4. 1. Globorotalia crater Finlay, Sample $516 \mathrm{~F}-77-5,63-66 \mathrm{~cm}$, side view, $\times 80$. 2. Globorotalia aragonensis Nuttal, Sample $516 \mathrm{~F}-78-4$, $78-80 \mathrm{~cm}$, umbilical view, $\times 70$. 3. Sample $516 \mathrm{~F}-80-3,88-90 \mathrm{~cm}, \times 90$, some aspect of the planktonic foraminifers with $\mathrm{H}_{2} \mathrm{~S}$ crystallized. $4-5$. Globigerina hornibrooki Brönnimann, $\times 70$, (4) Sample 516F-75-6, 99-101 cm, umbilical view, (5) Sample 516F-73-4, 148-150 cm, spiral view. 6-7. Sample 516F-75,CC, (6) Pseudohastigerina wilcoxensis (Cushman and Ponton), axial apertural view, $\times 160$, (7) Pseudohastigerina micra (Cole), axial apertural view, $\times 150$. 8. Globigerinita taroubaensis (Brönnimann), Sample 516F-67-2, 72-73 cm, umbilical view, $\times 105$. 9. Globigerina frontosa Subbotina, Sample 516F-62-3, 42-43 cm, side view, $\times 95$. 10-13. Sample 516F-76-3, 123-125 cm, (10-11) Globorotalia broedermanni Cushman and Bermudez, $\times 150,(10$, umbilical view; 11, spiral view), (12-13) Globorotalia prolata (Bolli), (12, spiral view, $\times 130 ; 13$, umbilical view, $\times 120$ ). 14-15. Globorotalia aspensis $($ Colom) Sample 516F-75,CC, (14) spiral view, $\times 120,(15)$ umbilical view, $\times 115$. 

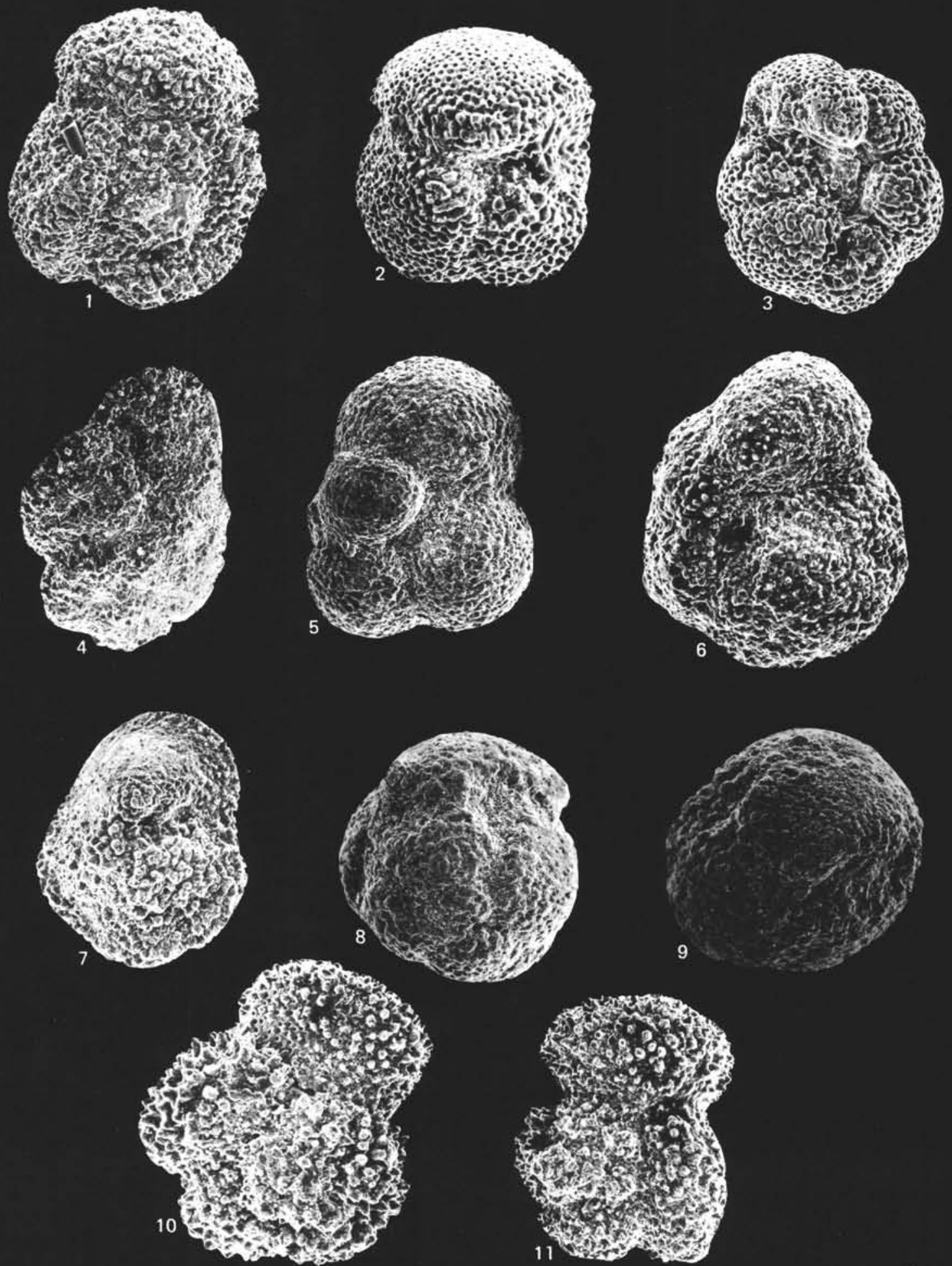

Plate 5. 1-2. Globorotalia bullbrooki Bolli, (1) Sample 516F-70-2, 75-77 cm, spiral view, $\times 100$, (2) Sample $516 \mathrm{~F}-73-4,148-150 \mathrm{~cm}$, umbilical view, $\times 125$, this specimen shows a relatively closed umbilicus. 3. Globorotalia pentacamerata Subbotina, Sample $516 \mathrm{~F}-73-4,148-150 \mathrm{~cm}$, umbilical view, $\times 110$, 4. Globorotalia ex. int, lehneri Cushman and Jarvis, Sample 516F-62-2, 69-71 cm, spiral view, $\times 90$. 5. Globigerinita turgida Finlay, Sample 516F-77-2, 29-31 cm, umbilical view, $\times 80$. 6. Globorotalia broedermanni Cushman and Bermudez, Sample 516F-63-3, 42-43 cm, umbilical view, $\times 125$. 7. Globorotalia spinulosa Cushman, Sample 516F-70-2, 75-77 cm, umbilical view, $\times 80$. 8-9. Globigerinatheka subconglobata (Shutskaya), Sample 516F-64,CC, spiral view, $(8) \times 70,(9) \times 90$. 10-11. Truncorotaloides topilensis (Cushman), Sample 516F-62-3, 42-43 cm, spiral view, (10) $\times 110$, (11) $\times 90$. 

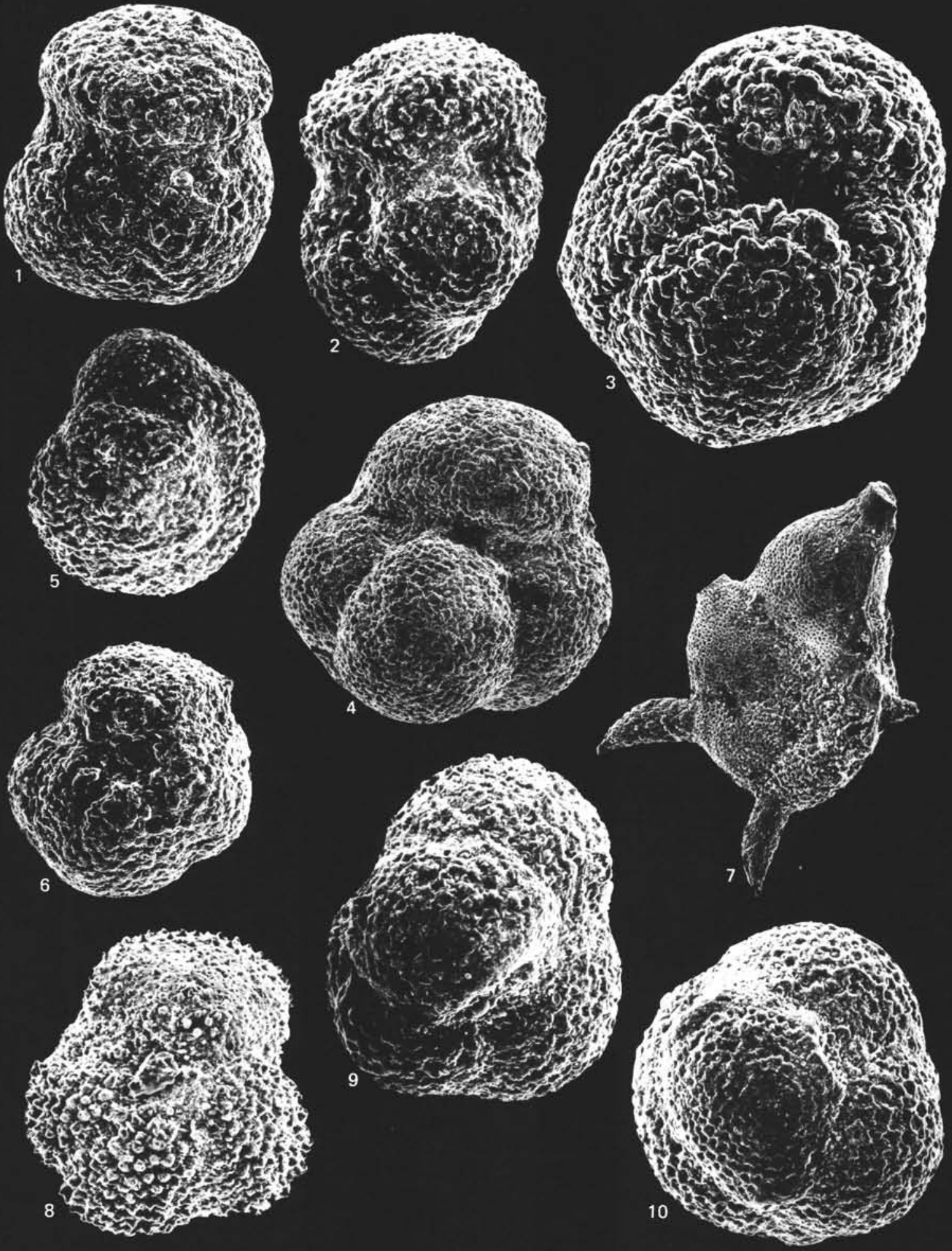

Plate 6. 1. Globigerina primitiva (Finlay), Sample $516 \mathrm{~F}-69-1,100-102 \mathrm{~cm}$, umbilical view, $\times 115$. 2. Globigerina triplex (Subbotina), Sample 516F-78-4, 78-80 cm, side view, $\times 155$. 3. Globigerinatheka senni (Beckmann), Sample 515B-56-4, 80-85 cm, umbilical view, $\times 180$. 4. Globigerina bakeri Cole, Sample $516 \mathrm{~F}-73-1,15-17 \mathrm{~cm}$, umbilical view, $\times 90$. 5. Globorotalia broedermanni Cushman and Bermudez, Sample $516 \mathrm{~F}-66-3,47-49 \mathrm{~cm}$, spiral view, $\times 125$. 6. Globorotalia $\mathrm{cf}$. bullbrooki Bolli, Sample 516F-65-2, 97-99 cm, umbilical view, $\times 115$. 7-8. Sample 516F-58,CC, (7) Hantkenina sp., side view, $\times 100$, (8) Globorotalia collactea Finlay, umbilical view, $\times 155$. 9. Globigerinita martini Blow and Banner, Sample 516F-56-1, 58-59 cm, umbilical view, $\times 170 . \quad 10$. Globigerinita globiformis Blow and Banner, Sample 516F-54-6, 53-55 $\mathrm{cm}$, umbilical view, $\times 160$. 

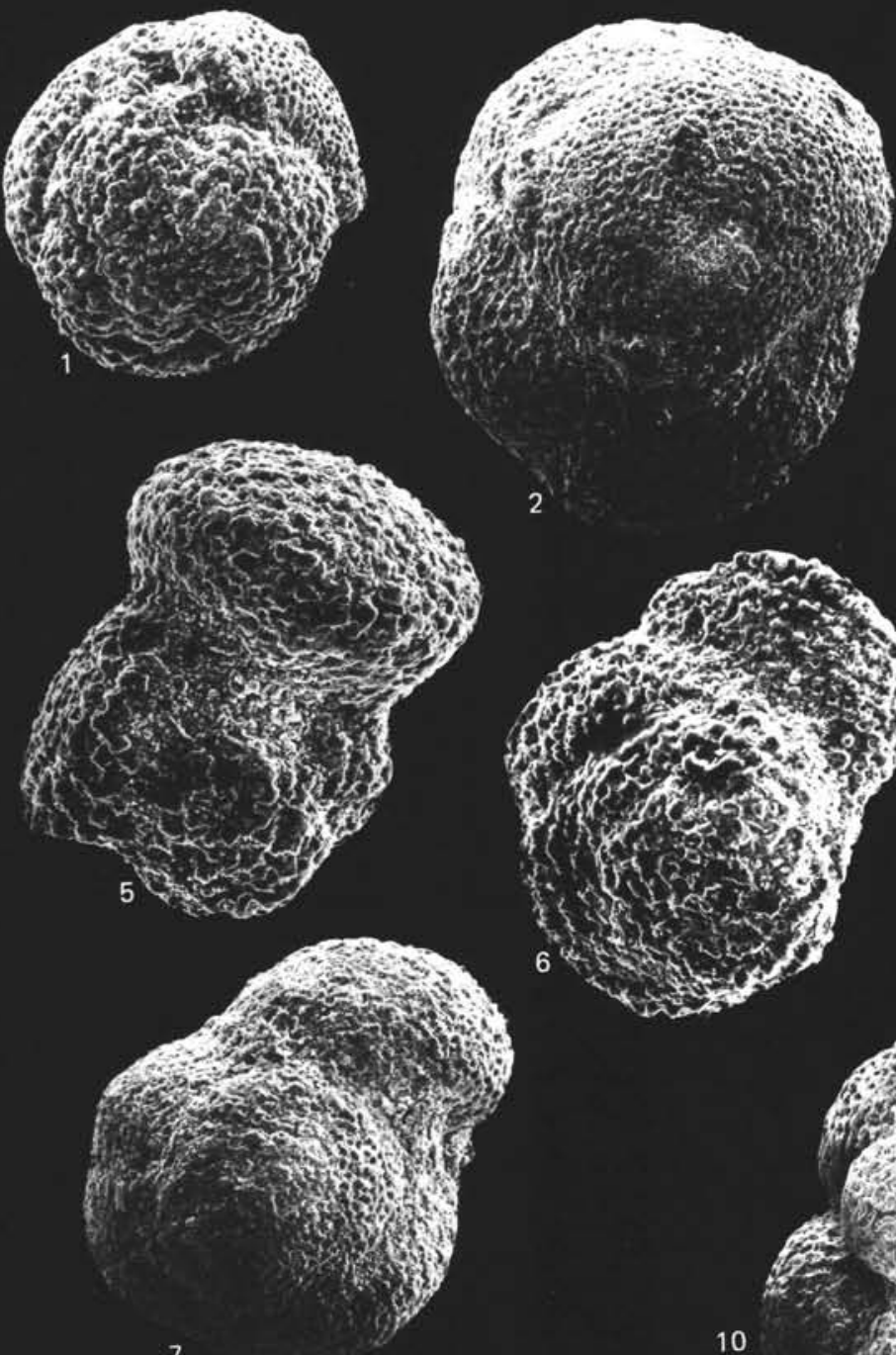

2
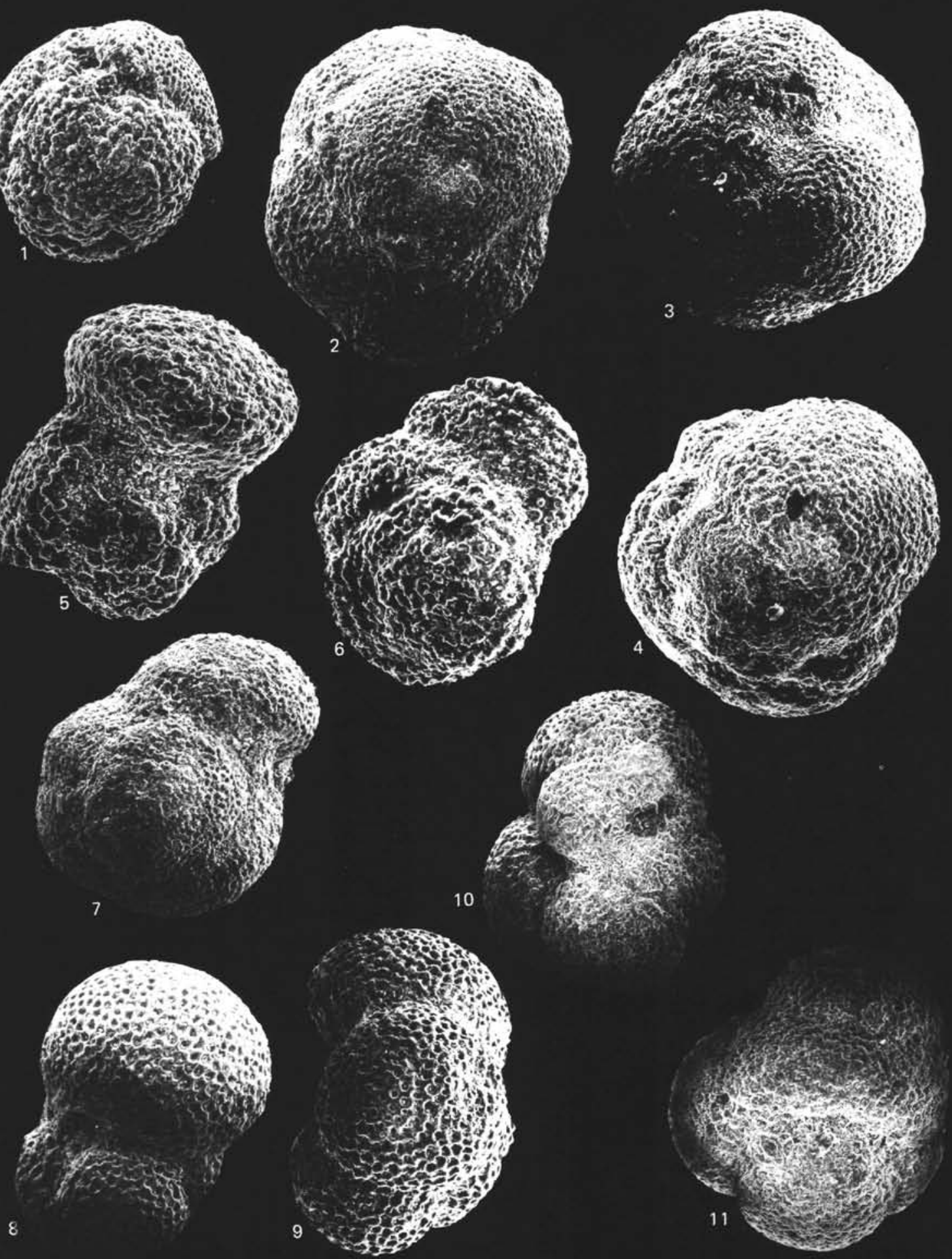

Plate 7. 1. Globigerinatheka mexicana (Cushman), Sample $516 \mathrm{~F}-62-3,42-44 \mathrm{~cm}$, spiral view, $\times 105$. 2-4. Sample $516 \mathrm{~F}-54-6$, $53-55 \mathrm{~cm},(2-3)$ Globigerinatheka luterbacheri Bolli, $\times 90$ (2, side view; 3, spiral view), (4) Globigerinatheka kugleri (Bolli, Loeblich, and Tappan), side view, $\times 95$. 5. Truncorotaloides topilensis (Cushman), Sample $516 \mathrm{~F}-53-4,29-31 \mathrm{~cm}$, umbilical view, $\times 125$. 6-7. Sample $516 \mathrm{~F}-53-3,29-31 \mathrm{~cm},(6)$ Globorotalia spinulosa Cushman, spiral view, $\times 140$, (7) Globigerina higginsi (Bolli), oblique spiral view, $\times 90$. 8. Globigerina linaperta Finlay, Sample 516F-50-1, 58-59 cm, umbilical view, $\times 125$. 9. Globigerina corpulenta Subbotina, Sample 516F-52-4, 61-63 cm, side view, $\times 105$. 10. Globigerinita simulans (Bermudez), Sample $516 \mathrm{~F}-51-2,25-27 \mathrm{~cm}$, umbilical view, $\times 80 . \quad 11$. Globigerinita pera (Todd), Sample $516 \mathrm{~F}-52-2,61-63 \mathrm{~cm}$, umbilical view, $\times 90$. 

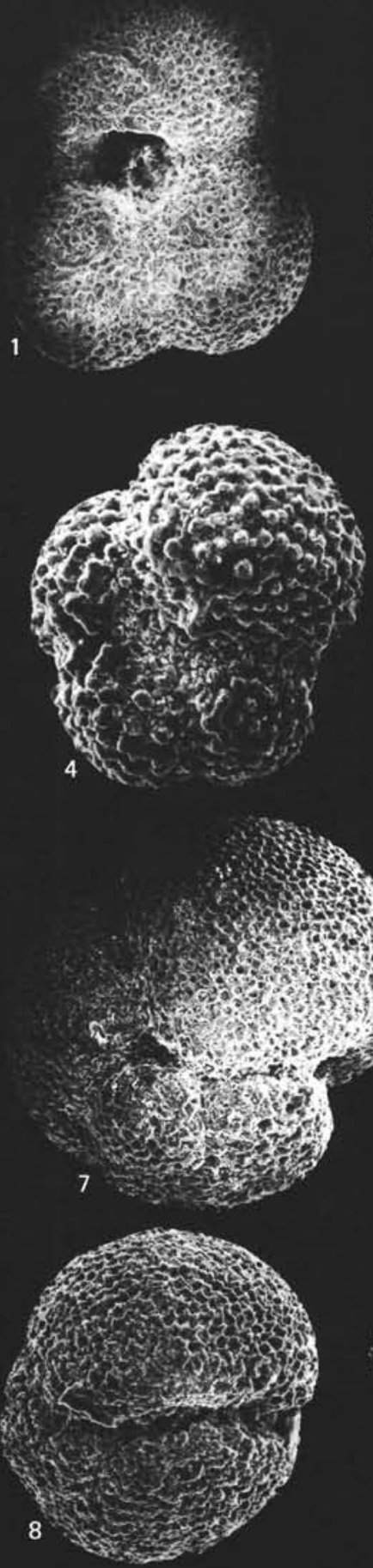
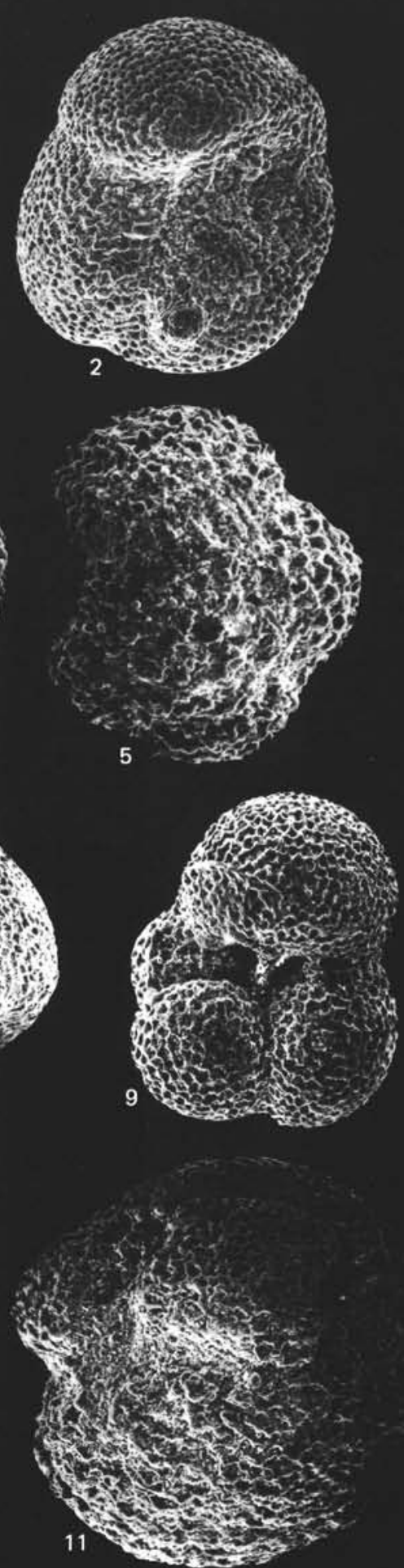
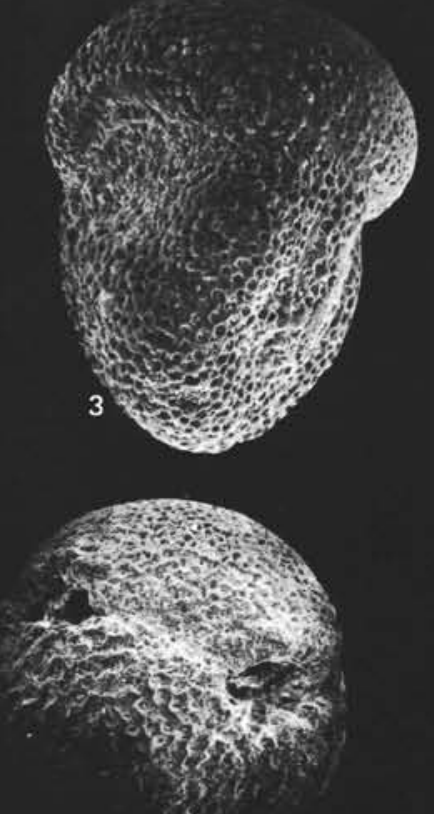

6
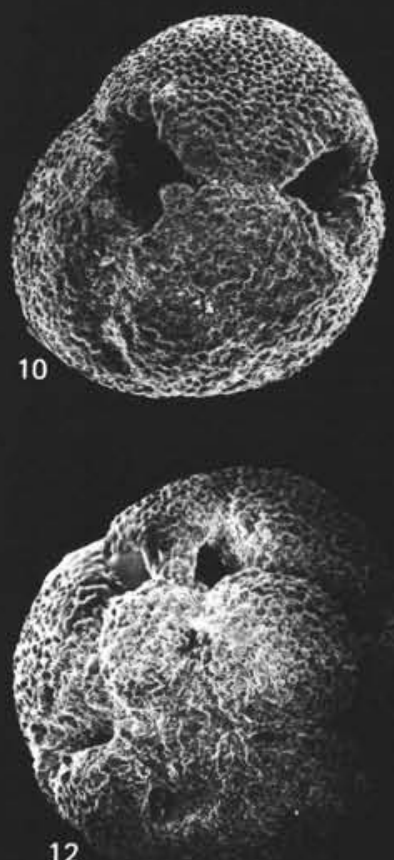

12

Plate 8. 1-3. Sample 516F-52-4, 61-63 cm, (1) Globigerina pseudoeocaena Subbotina, umbilical view, $\times 95$, (2) Globorotalia possagnoensis Toumarkine and Bolli, $\times 105$, (3) Globorotalia pomeroli Toumarkine and Bolli, $\times 115$. 4-5. Globorotalia cf. medizzai (Toumarkine and Bolli), Sample 516F-49-1, 83-84 cm, $\times 175$, (4) umbilical view, (5) spiral view. 6. Globigerinita howei Blow and Banner, Sample 516F-48-1, 63-64 cm, oblique umbilical view, $\times 80$. 7-8. Globigerinatheka semiinvoluta spp., (7) Sample $516 \mathrm{~F}-41-1,79-80 \mathrm{~cm}$, spiral view, $\times 80,(8)$ Sample $516 \mathrm{~F}-45-1,43-45 \mathrm{~cm}$, oblique umbilical view, $\times 90$. 9. Globigerina galavisi Bermudez, Sample $516 \mathrm{~F}-45-1,43-45 \mathrm{~cm}$, umbilical view, $\times 90$. 10-11. Sample 516F-41-1, 79-80 cm, (10) Globigerinatheka tropicalis (Blow and Banner), side view, $\times 60$, (11) Globigerinatheka luterbacheri Bolli, side view, $\times 90$. 12. Globigerinatheka barri Brönnimann, Sample 516F-40-1, 104-106 cm, distal view, $\times 60$. 

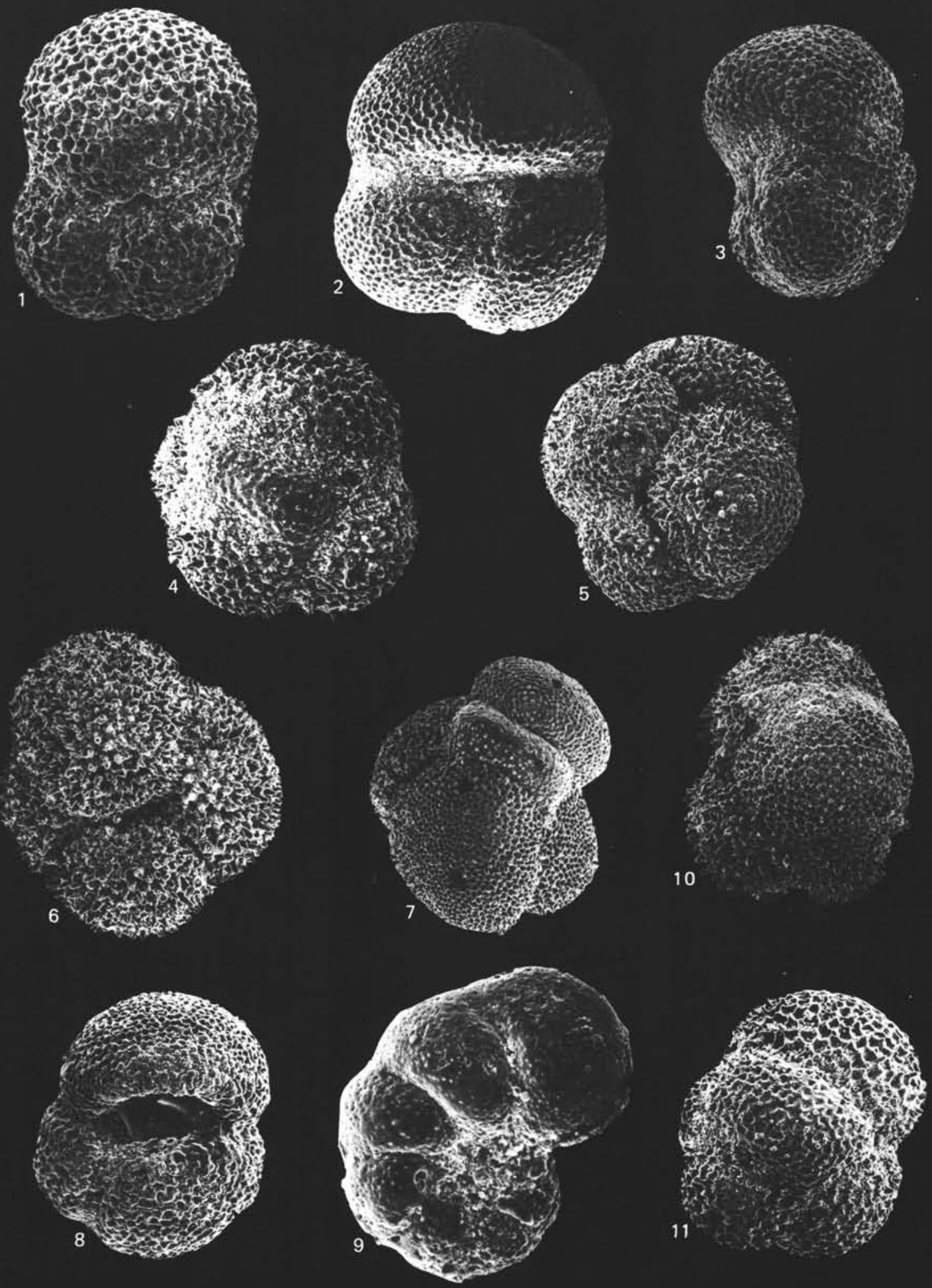

Plate 9. 1. Globigerina linaperta Finlay, Sample 516F-45-1, 43-45 cm, umbilical view, $\times 150.2-3$. Sample 516-42-1, 50-52 cm, $\times 85,(2)$ Globigerina tripartita Koch, umbilical view, (3) Globigerina sellii Borsetti, side view. 4-7. Globoquadrina? spiny form, Sample 516-42,CC, umbilical view, (4) $\times 110$, (5) $\times 85,(6) \times 75$, (7) $\times 65$. 8. Globigerinatheka index Finlay, Sample $516 \mathrm{~F}-40-1,104-106 \mathrm{~cm}$, umbilical view, $\times 95$. 9 . Pseudohastigerina barbadoensis Blow, Sample 516F-40-2, 100-102 cm, side view, $\times 180$. 10. Globoquadrina? spiny form, Sample 516-38,CC, umbilical view, $\times 85$. 11. Globigerinita riveroae Bermudez, Sample $516-35-1,60-62 \mathrm{~cm}$, umbilical view, $\times 85$. 

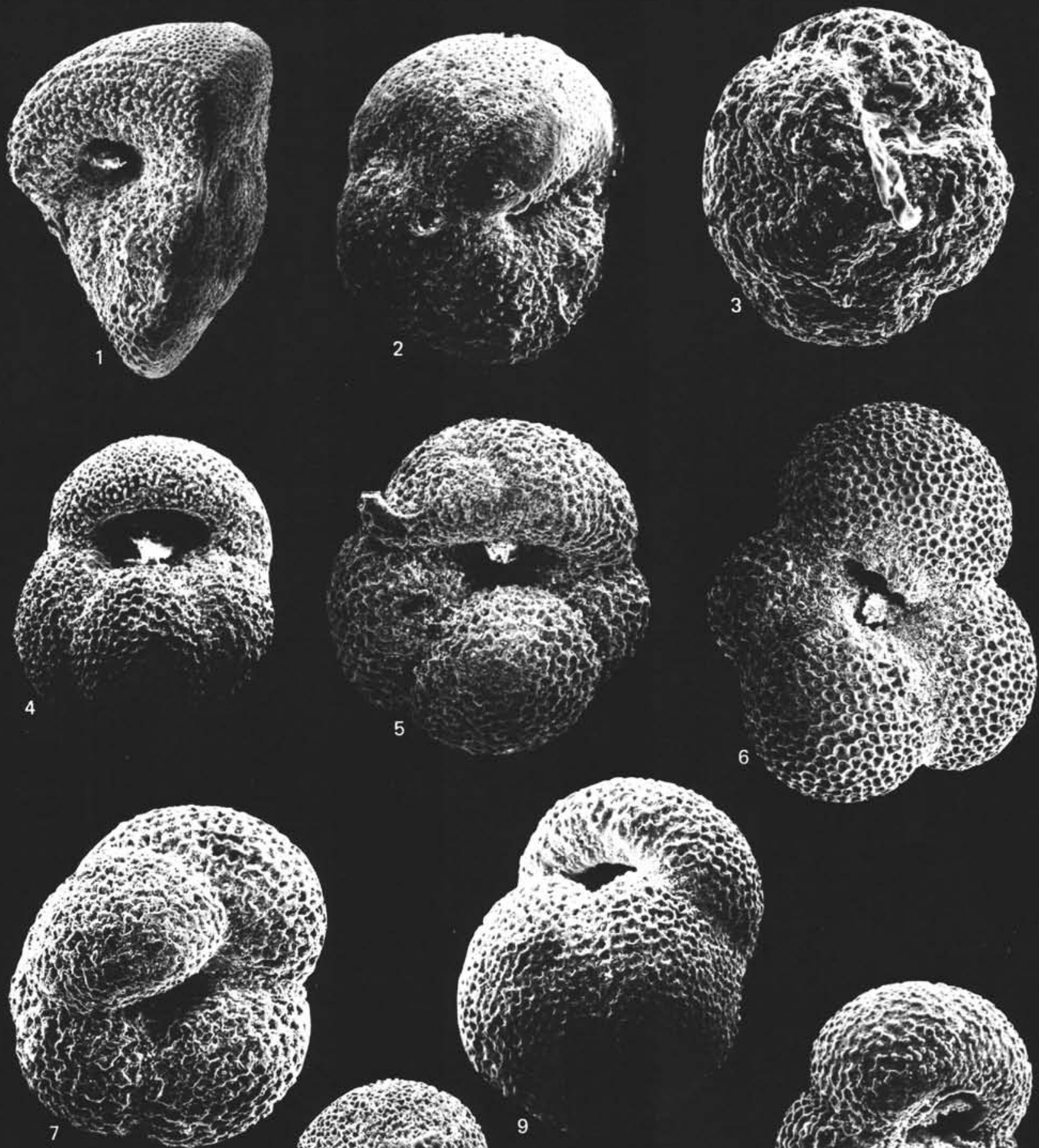

5

6
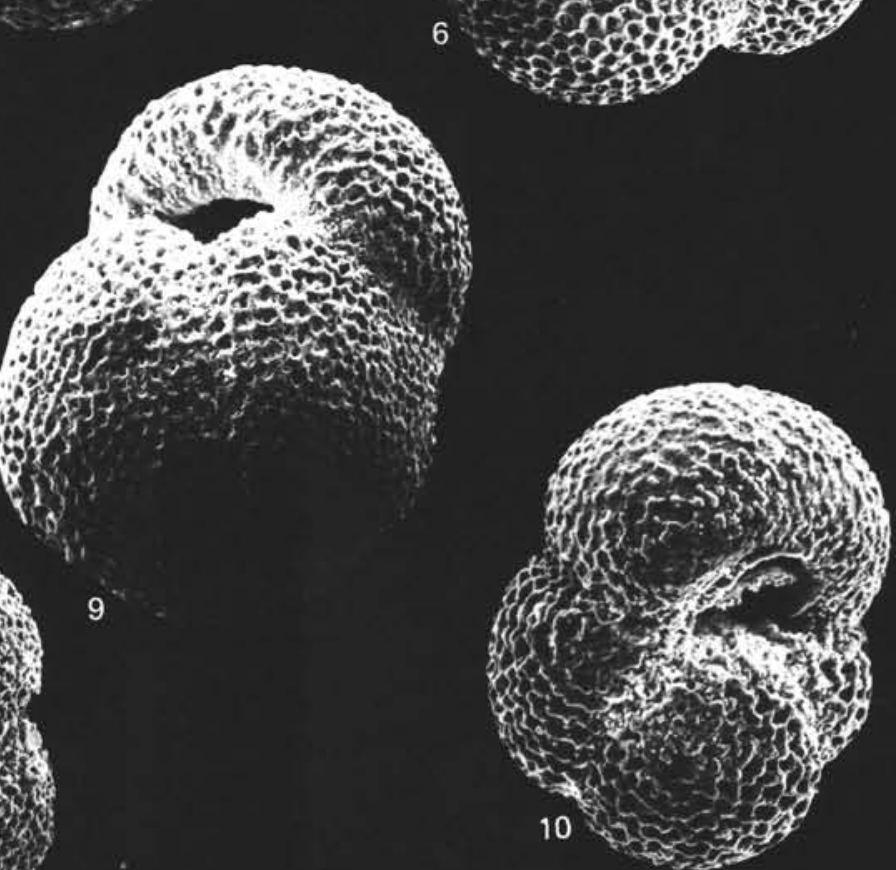

Plate 10. 1. Globorotalia cerroazulensis (Cole), Sample $516 \mathrm{~F}-40-1,104-106 \mathrm{~cm}$, side view, $\times 105$. 2. Globorotalia cocoaensis Cushman, Sample $516 \mathrm{~F}-39, \mathrm{CC}$, umbilical view, $\times 125$. 3. Globigerinita africana Blow and Banner, Sample 516F-39-2, 6-7 cm, umbilical view, $\times 155.4,8,10$. Globigerina ampliapertura Bolli, $(4,8)$ Sample 516F-34,CC; umbilical view, $\times 95$, (10) Sample 516F-29-3, 90-91 cm, umbilical view, $\times 155$. 5 . Globigerina euapertura Jenkins, Sample 516F-33-5, 31-32 cm, umbilical view, $\times 115$. 6. Globigerina galavisi Bermudez, Sample 516F-32-4, $36-38 \mathrm{~cm}$, umbilical view, $\times 85$. 7. Catapsydrax unicava primitiva (Blow and Banner), Sample $516 \mathrm{~F}-30-5,33-35 \mathrm{~cm}, \mathrm{umbilical}$ view, $\times 105$. 9 . Globigerina praeturritilina Blow and Banner, Sample 516F-30-4, 31-33 cm, oblique umbilical view, $\times 105$. 

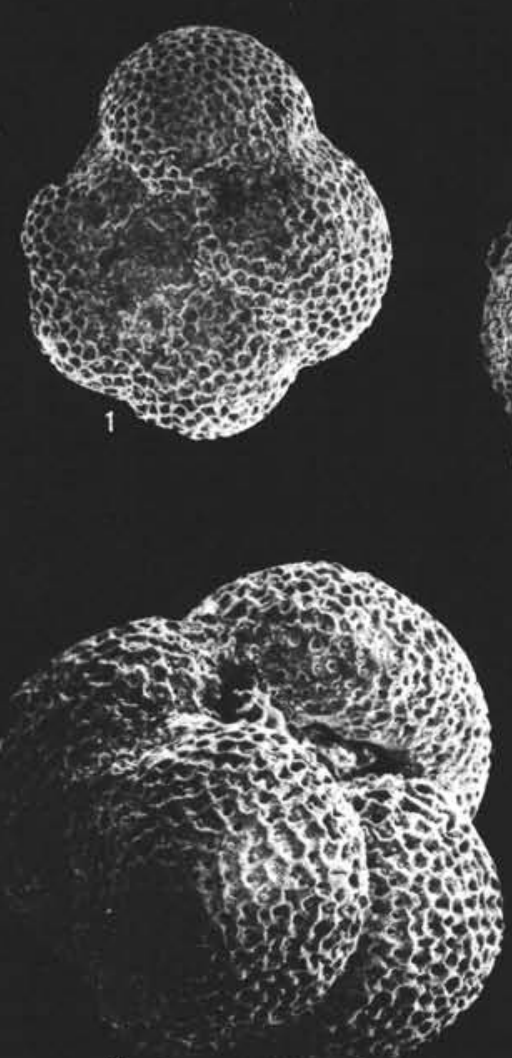

4
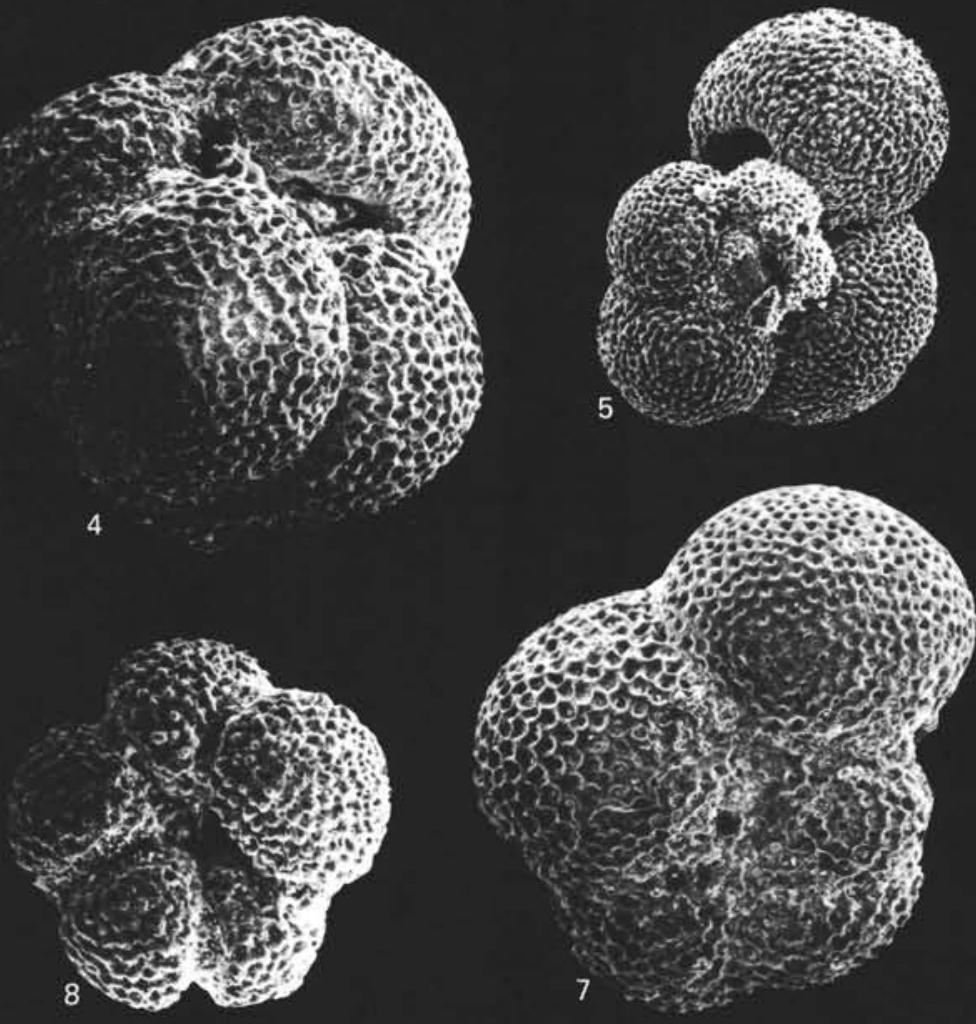
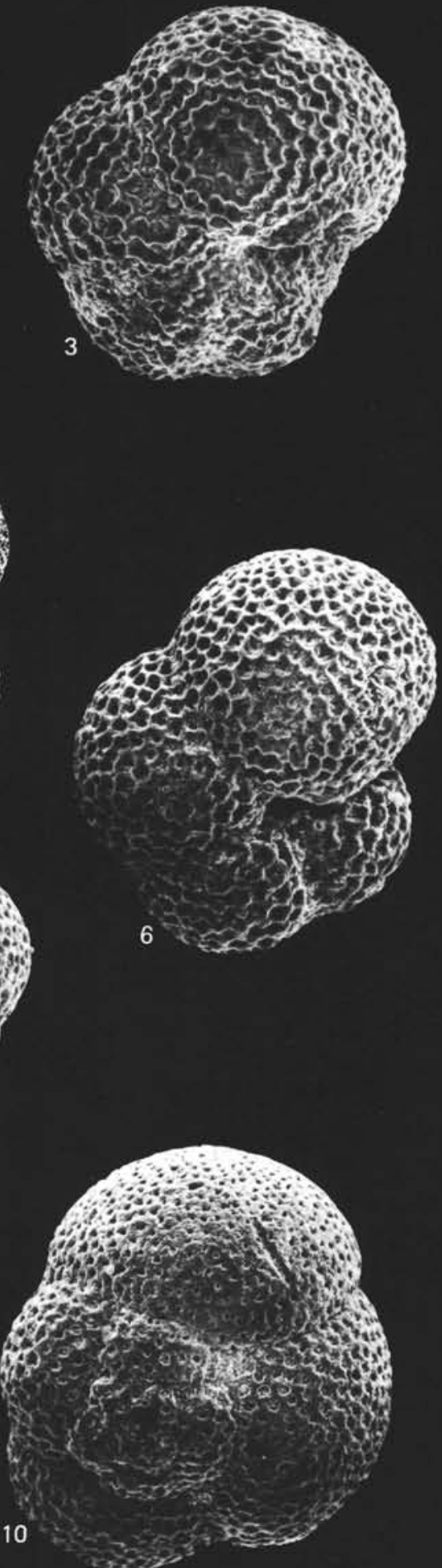

Plate 11. 1. Globorotalia siakensis Le Roy, Sample 516F-30-6, 33-35 cm, spiral view, $\times 120$. 2-3. Sample 516F-27-3, 13-15 cm, (2) Globigerina angiporoides Hornibrook, umbilical view, $\times 100,(3)$ Globorotalia continuosa Blow, umbilical view, $\times 185$. 4. Globigerina gortanii Borśetti, Sample 516F-27-1, 89-90 cm, oblique umbilical view, $\times 110$. 5. Globorotalia obesa Bolli, Sample 516-25,CC, spiral view, $\times 100$. 6. Globorotalia opima nana Bolli, Sample 516F-25-3, 31-32 cm, umbilical view, $\times 145$. 7. Globorotalia opima opima Bolli, Sample 516F-24-1, 3-5 cm, spiral view, $\times 140$. 8. Globigerina anguliofficinalis Blow, Sample $516 \mathrm{~F}-23, \mathrm{CC}$, umbilical view, $\times 165$. 9. Globigerina senilis Bandy, Sample $516 \mathrm{~F}-25-4,89-91 \mathrm{~cm}$, umbilical view, $\times 145$. 10. Globigerina euapertura Jenkins, Sample 516F-20,CC, spiral view, $\times 110$. 


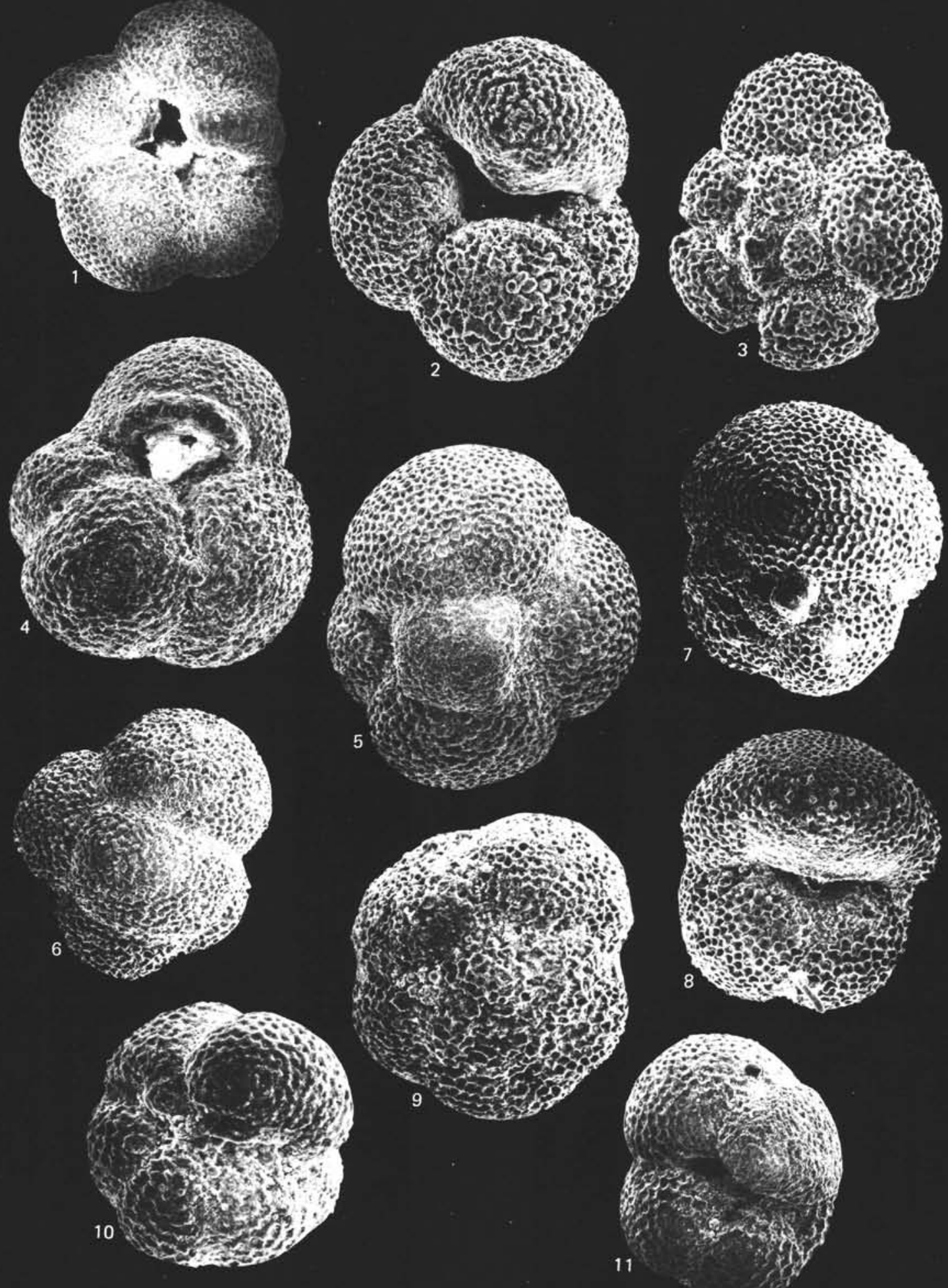

Plate 12. 1. Globoquadrina globularis Bermudez, Sample 516F-21-4, 60-62 cm, umbilical view, $\times 90$. 2. Globoquadrina baroemoensis (Le Roy), Sample 516F-20,CC, umbilical view, $\times 95$. 3. Globigerina angulisuturalis Bolli, Sample 516F-20-5, $50-52 \mathrm{~cm}$, spiral view, $\times 175$. 4 . Globigerina labiacrassata Jenkins, Sample 516F-20-3, 50-52 cm, umbilical view, $\times 115$. 5. Catapsydrax dissimilis dissimilis Cushman and Bermudez, Sample $516 \mathrm{~F}-15-6,50-52 \mathrm{~cm}$, umbilical view, $\times 90$. 6. Globigerinita riveroae Bermudez, Sample $516 \mathrm{~F}-15-1,50-52 \mathrm{~cm}$, umbilical view, $\times 70$. 7-10. Sample 516F-13-3, 77-81 cm, (7-8) Globigerina binaiensis Koch, (7, spiral view, $\times 115 ; 8$, umbilical view, $\times 105)$, (9-10) Globorotalia mendacis Blow, $(9$, spiral view, $\times 175 ; 10$, umbilical view, $\times 195)$. 11. Globorotaloides suteri Bolli, Sample $516 \mathrm{~F}-12-1,19-20 \mathrm{~cm}$, umbilical view, $\times 80$. 

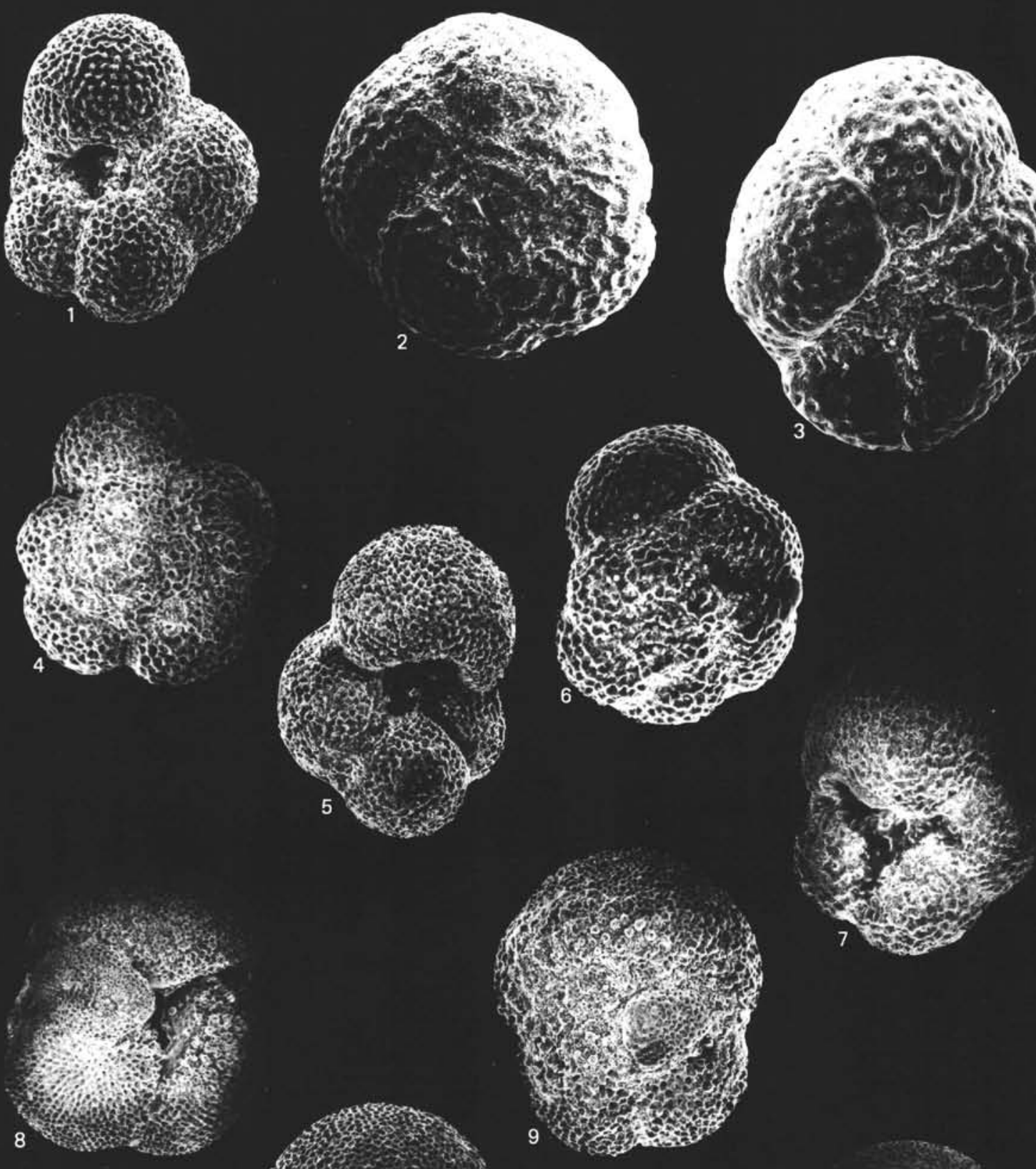

10
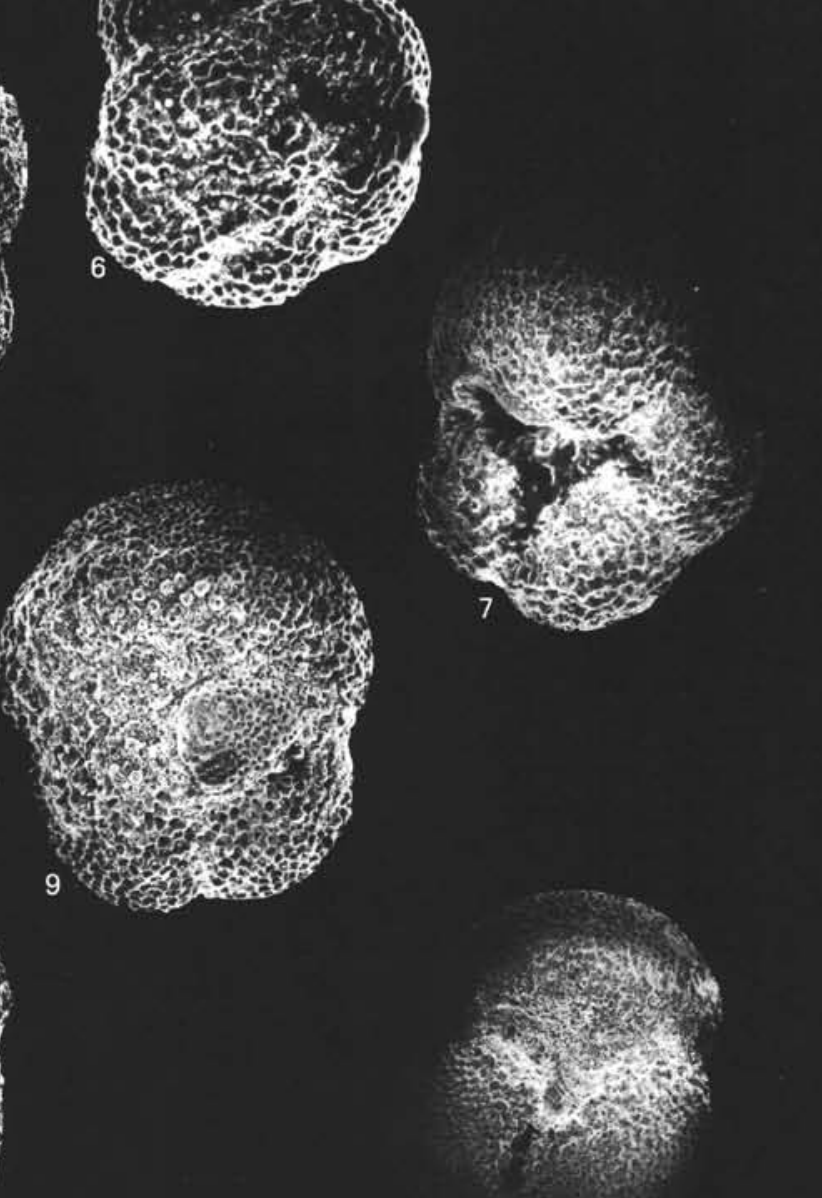

11

Plate 13. 1. Globigerina ciperoensis Bolli, Sample 516F-21,CC, umbilical view, $\times 120$. 2-3. Globorotaloides testarugosa Jenkins, Sample $516 \mathrm{~F}-11-3,102-103 \mathrm{~cm},(2)$ spiral view, $\times 135$, (3) umbilical view, $\times 145$. 4, 6. Sample 516F-11-2, $16-17 \mathrm{~cm}$, (4) Globigerina angustiumbilicata Bolli, spiral view, $\times 145$, (6) Globorotalia mendacis Blow, spiral view, $\times 155$. 5. Globigerina praebulloides Blow, Sample $516 \mathrm{~F}-11, \mathrm{CC}$, umbilical view, $\times 90$. 7. Globorotalia continuosa Blow, Sample 516F-10-2, 50-52 cm, umbilical view, $\times 120$. 8-11. Sample 516F-5, CC, (8) Globoquadrina praedehiscens Blow and Banner, umbilical view, $\times 65$, (9) Globigerina sellii Borsetti, spiral view, $\times 70$, (10-11) Globigerina tripartita Koch (10, umbilical view, $\times 70 ; 11$, umbilical view, $\times 45)$. 

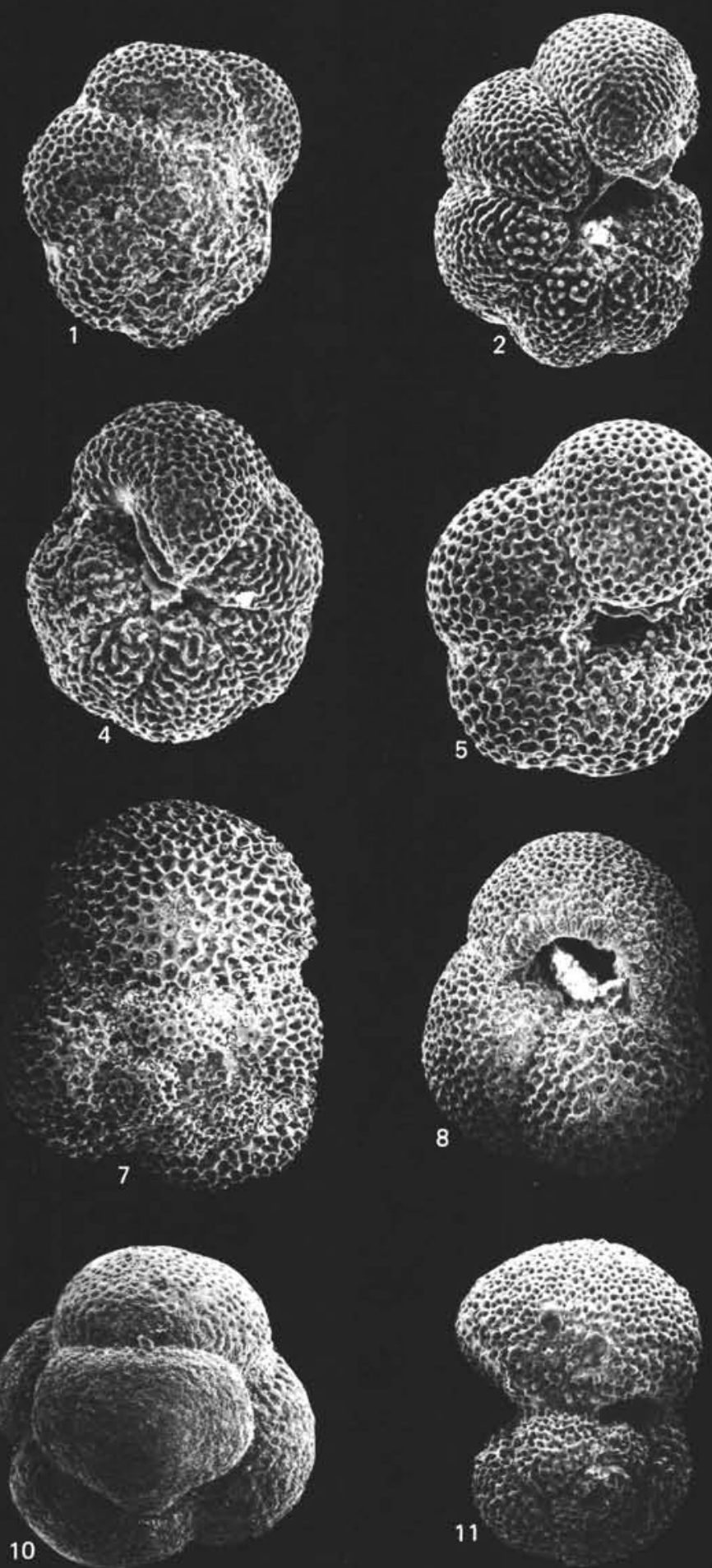
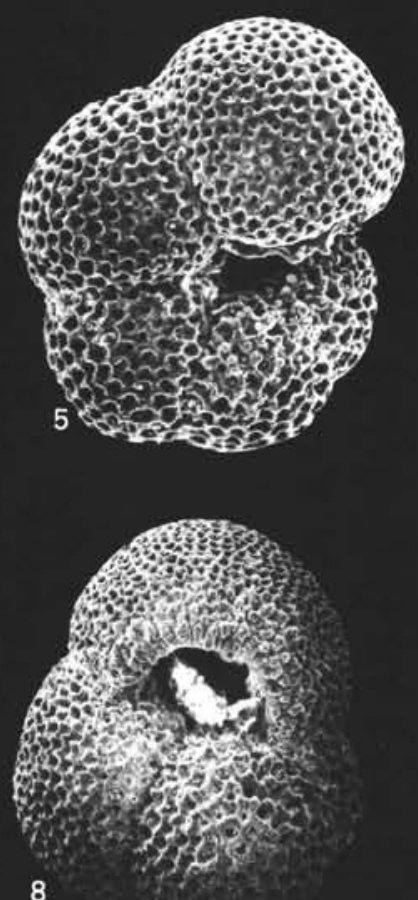

8

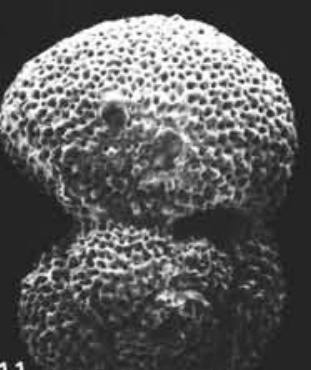

11

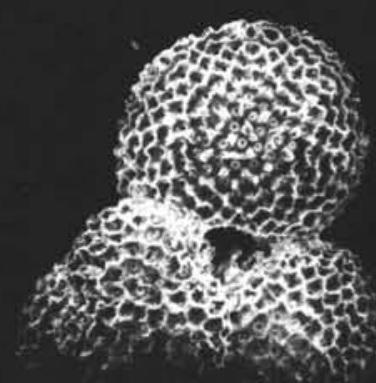

3
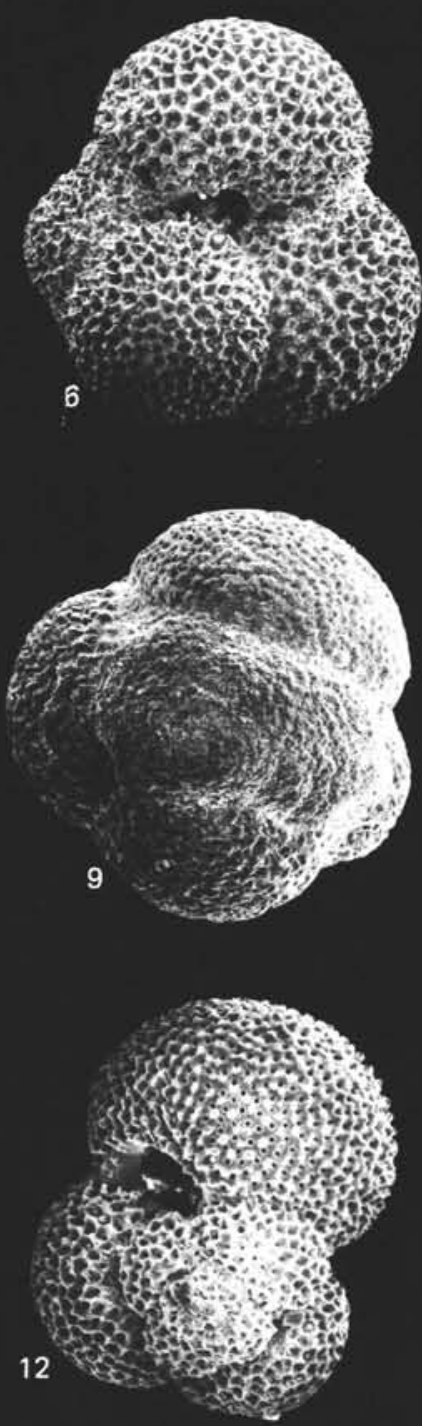

Plate 14. 1-2. Globorotalia kugleri Bolli, (1) Sample 516F-2-2, 50-52 cm, spiral view, $\times 135$, (2) Sample 516F-2,CC, umbilical view, $\times 145$, this specimen can be referred to G. pseudokugleri Blow. 3. Globigerinoides primordius Blow and Banner, Sample $516 \mathrm{~F}-2, \mathrm{CC}$, spiral view, $\times 120$. 4-5. Sample 516-43,CC, umbilical view, $\times 135$, (4) Globorotalia mendacis Blow, (5) Globorotalia siakensis Le Roy. 6-7. Globigerina galavisi Bermudez, Sample 516-42,CC, (6) umbilical view, $\times 70$, (7) spiral view, $\times 90$. 8. Globigerina labiacrassata Jenkins, Sample 516-39,CC, umbilical view, $\times 70$. 9-10. Catapsydrax dissimilis ciperoensis Blow and Banner, Sample 516-39-1, 60-62 cm, umbilical view, (9) $\times 80$, (10) $\times 70$. 11-12. Globigerinoides altiaperturus Bolli, (11) Sample 516-38,CC, spiral view, $\times 80,(12)$ Sample $516-34, C C$, spiral view of a specimen growth to Globigerinoides ruber, $\times 100$. 

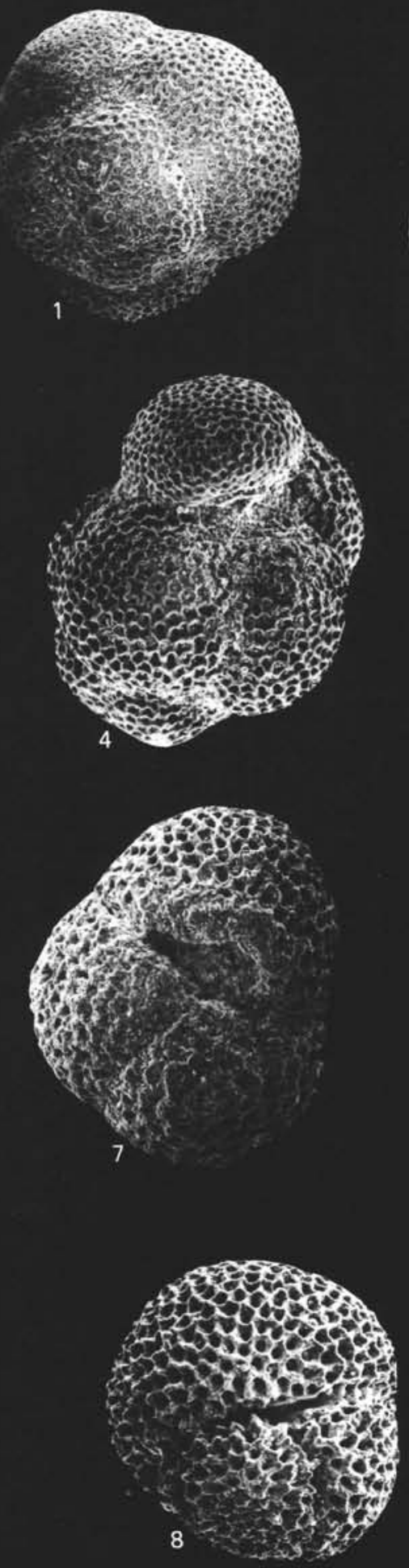
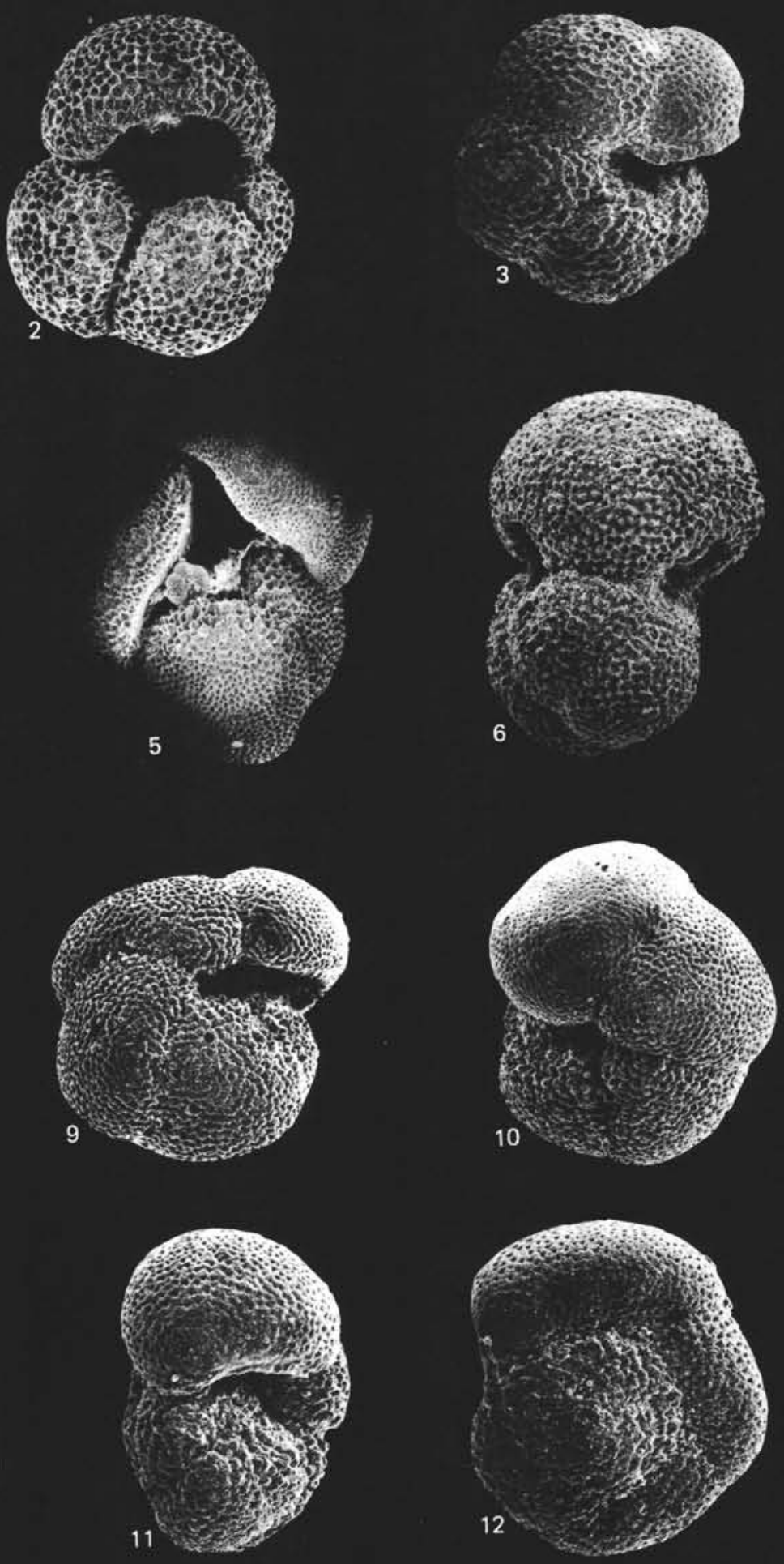

Plate 15. 1. Globigerina venezuelana Hedberg, Sample 516-35-3, 30-32 cm, spiral view, $\times 80$. 2-3. Sample 516-34-2, 90-92 cm, $\times 90$, (2) Globigerina woodi Jenkins, umbilical view, (3) Globorotalia continuosa Blow, umbilical view. 4. Globigerina gortanii Borsetti, Sample 516-33-2, $90-92 \mathrm{~cm}$, umbilical view, $\times 100$. 5. Globoquadrina baroemoenensis (Le Roy), Sample 516-32-2, 35-37 cm, umbilical view, $\times 65$. 6-7. Sample 516-30-4, 15-17 cm, (6) Globigerinoides altiaperturus Bolli, side view, $\times 75$, (7) Globigerina labiacrassata Jenkins, umbilical view, $\times 100$. 8-12. Sample 516-25,CC, (8) Globigerinoides sicanus de Stefani, umbilical view, $\times 90,(9-12)$ Globorotalia zealandica Hornibrook (9, umbilical view, $\times 80 ; 10$, umbilical view, $\times 80 ; 11$, side view, $\times 90 ; 12$, spiral view, $\times 110$ ). 

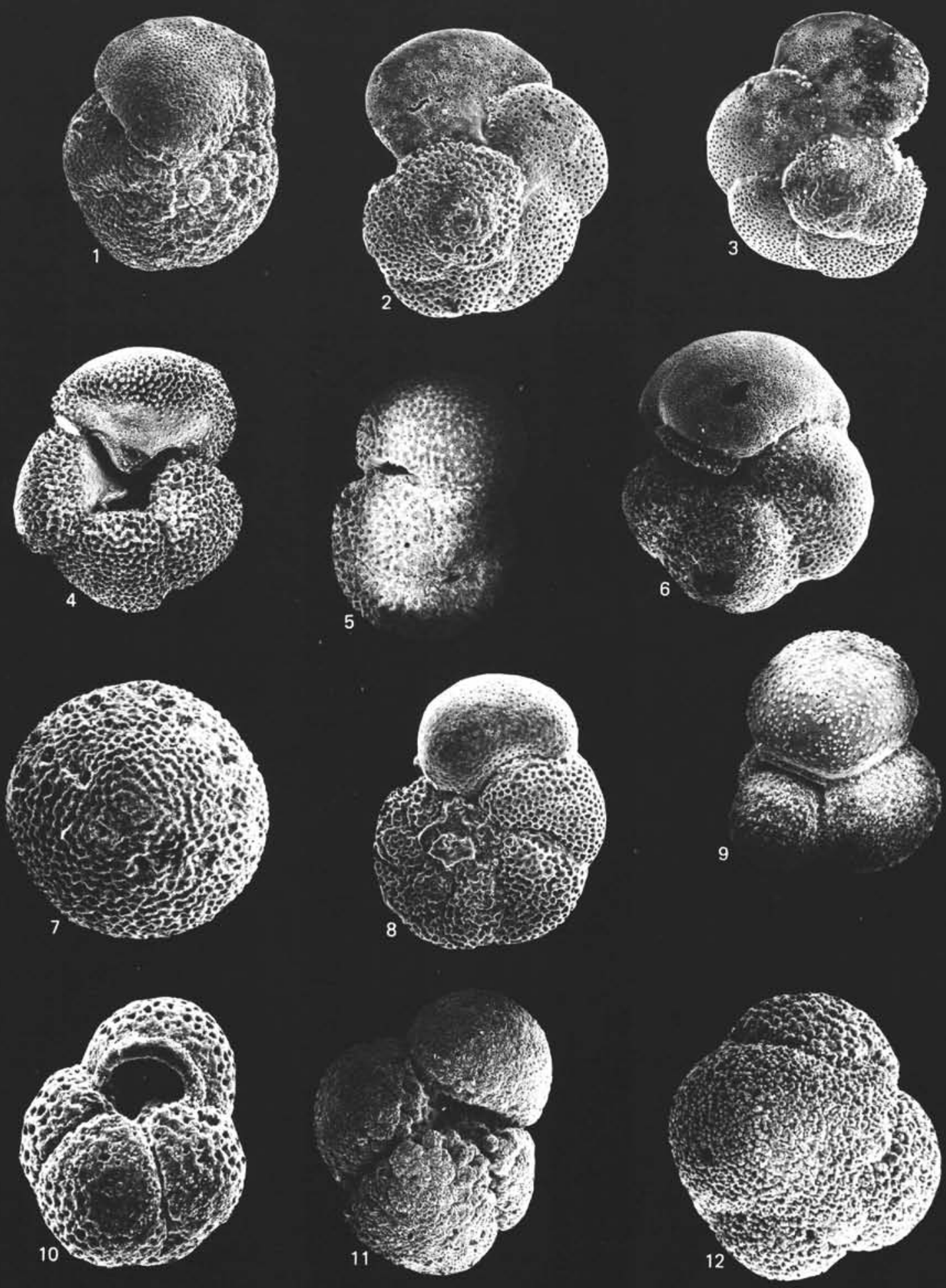

Plate 16. 1. Globorotalia miozea Finlay, Sample 516-22-1, 80-82 cm, umbilical view, $\times 70$. 2-3. Globorotalia praemenardii Cushman and Stainforth, (2) Sample 516-22-1, 80-82 cm, spiral view, $\times 90$, (3) Sample 516-21,CC, spiral view, $\times 80.4$, 6. Sample 516-21,CC, (4) Globoquadrina dehiscens (Chapman, Parr, and Collins), umbilical view, $\times 65,(6)$ Globorotalia scitula (Brady), umbilical view, $\times 120$. 5. Praeorbulina transitoria (Blow), Sample 516-21-2, 50-52 cm, oblique spiral view, $\times 70$. 7. Praeorbulina sp., Sample 516-18-2, 50-52 cm, spiral view, $\times 120$. 8. Globorotalia peripheroronda Blow and Banner, Sample $516-17-3,50-52 \mathrm{~cm}$, spiral view, $\times 100$. 9-12. Sample $516-15, \mathrm{CC},(9-10)$ Globigerinita glutinata Egger, umbilical view $(9, \times 125 ; 10, \times 135)$, (11) Sphaeroidinellopsis seminulina (Schawger), umbilical view, $\times 80,(12)$ Globigerina druryi Akers, umbilical view, $\times 145$. 

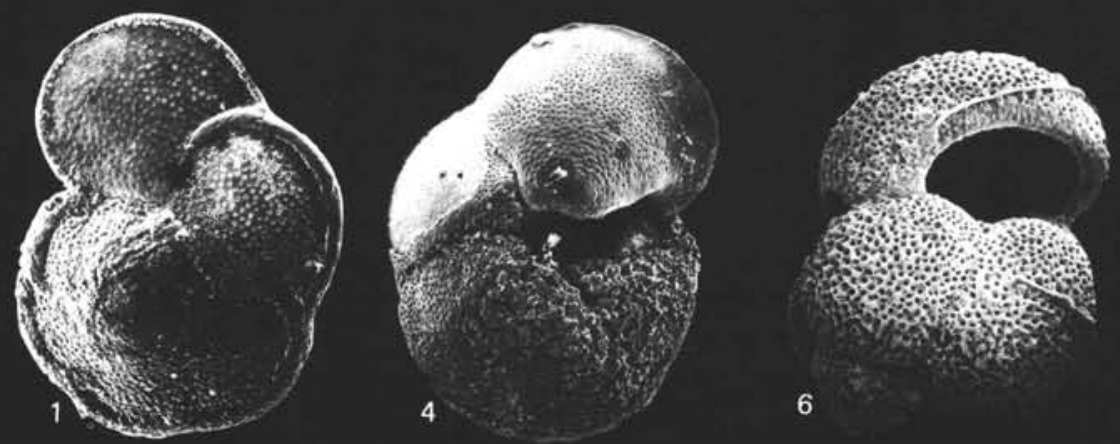

4

6
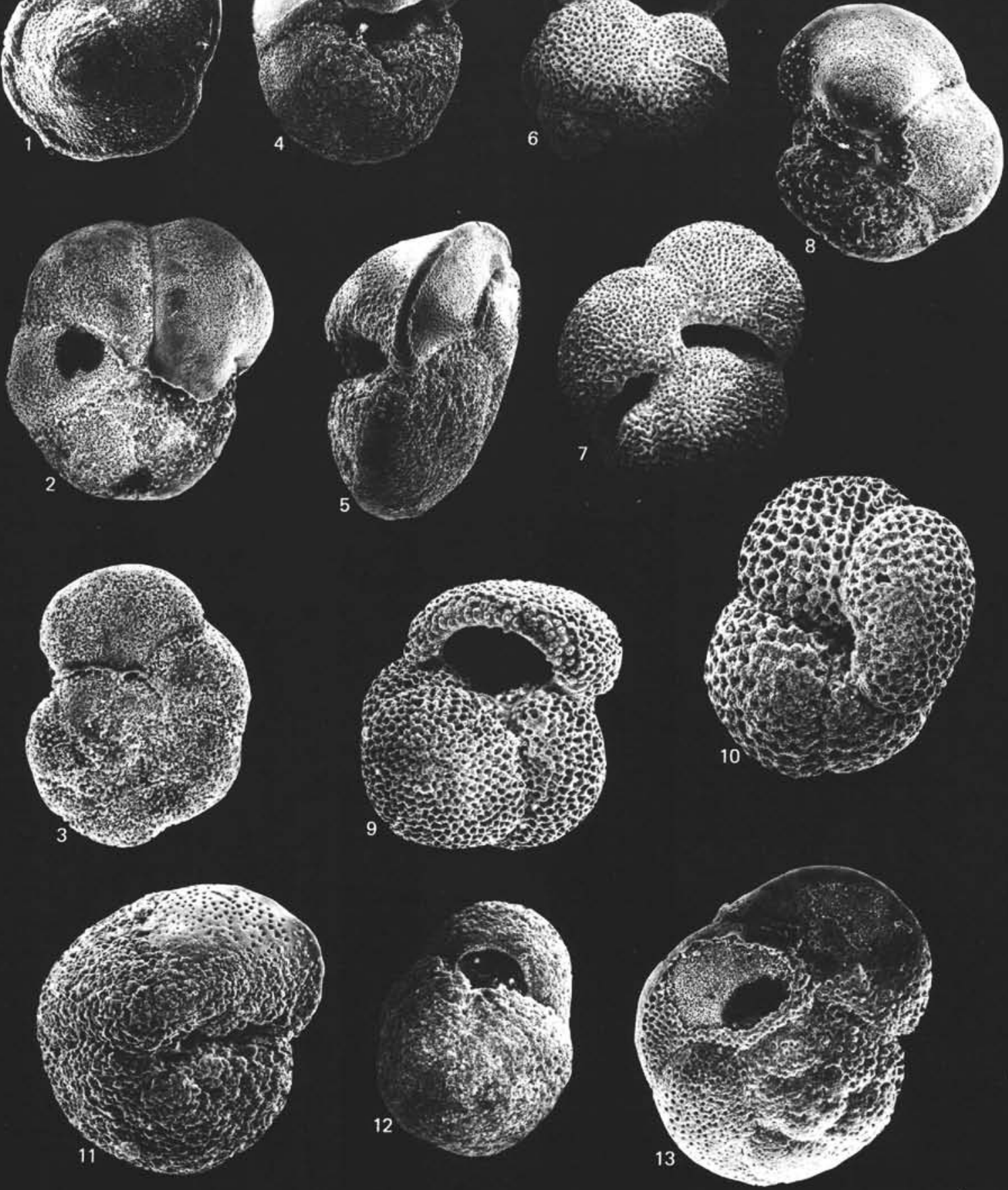

Plate 17. 1-3. Sample 516-14-1, 60-62 cm, (1) Globorotalia menardii s.1. (Parker, Jones, and Brady) ex d'Orbigny, spiral view, $\times 80$, (2-3) Globorotalia lenguaensis Bolli (2, umbilical view, $\times 110 ; 3$, spiral view, $\times 135)$. 4-5. Globorotalia conoidea Walters, Sample 516-13-2, 90-92 cm, (4) umbilical view, $\times 45,(5)$ side view, $\times 65$. 6-7, 9-12. Sample 516-11,CC, (6-7) Globigerinoides seigliei Bermudez and Bolli, (6, oblique umbilical view, $\times 65 ; 7$, distal view, $\times 55)$, (9) Globigerinoides extremus Bolli and Bermudez, umbilical view, $\times 90$, (10) Globigerinoides sacculiferus (Brady), umbilical view, $\times 90,(11)$ Globorotalia sphericonomiozea Walters, umbilical view, $\times 110,(12)$ Globigerina nepenthes Todd, umbilical view, $\times 110$. 8. Globorotalia scitula (Brady), Sample 516-12-3, 50-52 cm, umbilical view, $\times 115$. 13. Globorotalia cibaoensis Bermudez, Sample $516-10, C C$, spiral view, $\times 125$. 

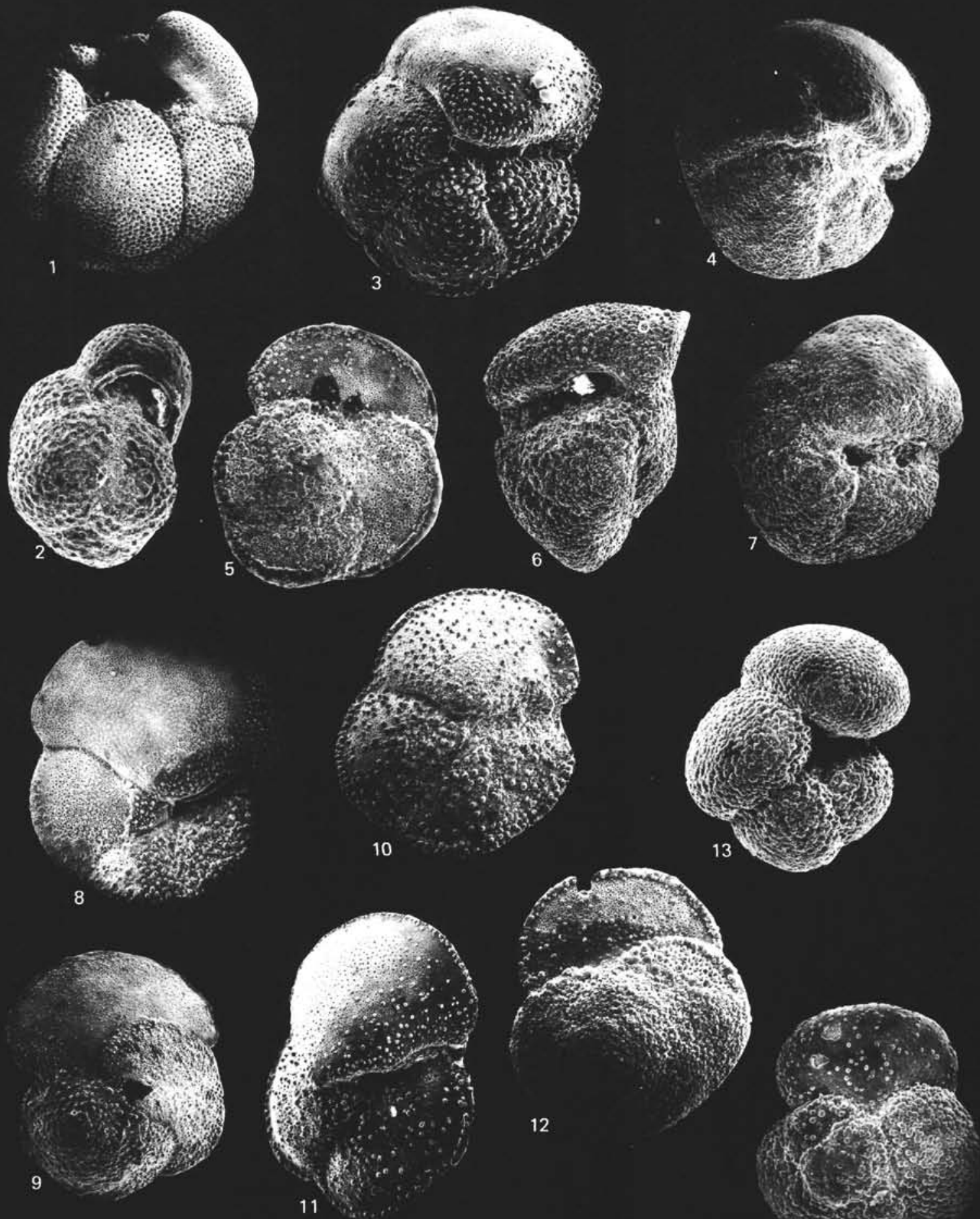

14

Plate 18. 1, 7. Sample 516-9-1, 70-72 cm, (1) Globoquadrina altispira (Cushman and Jarvis), umbilical view, $\times 45$, (7) Globorotalia crassaformis Galloway and Wissler, umbilical view, $\times 90$. 2. Globigerina nepenthes Todd, Sample 516-9,CC, umbilical view, $\times 80$. 3-6. Globorotalia conomiozea Kennett, (3) Sample 516A-8-2, 50-52 cm, umbilical view, $\times 70,(4,6)$ Sample 516-8,CC $(4$, umbilical view, $\times 60 ; 6$, side view, $\times 80)$, (5) Sample 516-9-1, 70-72 cm, spiral view, $\times 70$. 8-9. Globorotalia margaritae Bolli and Bermudez, (8) Sample 516-7,CC, umbilical view, $\times 80$, (9) Sample 516A-6-2, 50-52 cm, spiral view, $\times 65$. 10-12. Globorotalia crassula Cushman and Stewart, Sample 516A-7-2, 50-52 cm, (10) umbilical view, $\times 70$, (11) oblique umbilical view, $\times 70,(12)$ spiral view, $\times 65$. 13. Globorotalia crassaformis Galloway and Wissler, Sample $516 \mathrm{~A}-8-2,50-52 \mathrm{~cm}$, umbilical view, $\times 70$. 14. Globorotalia puncticulata (Deshayes), Sample 516A-8,CC, spiral view, $\times 80$. 


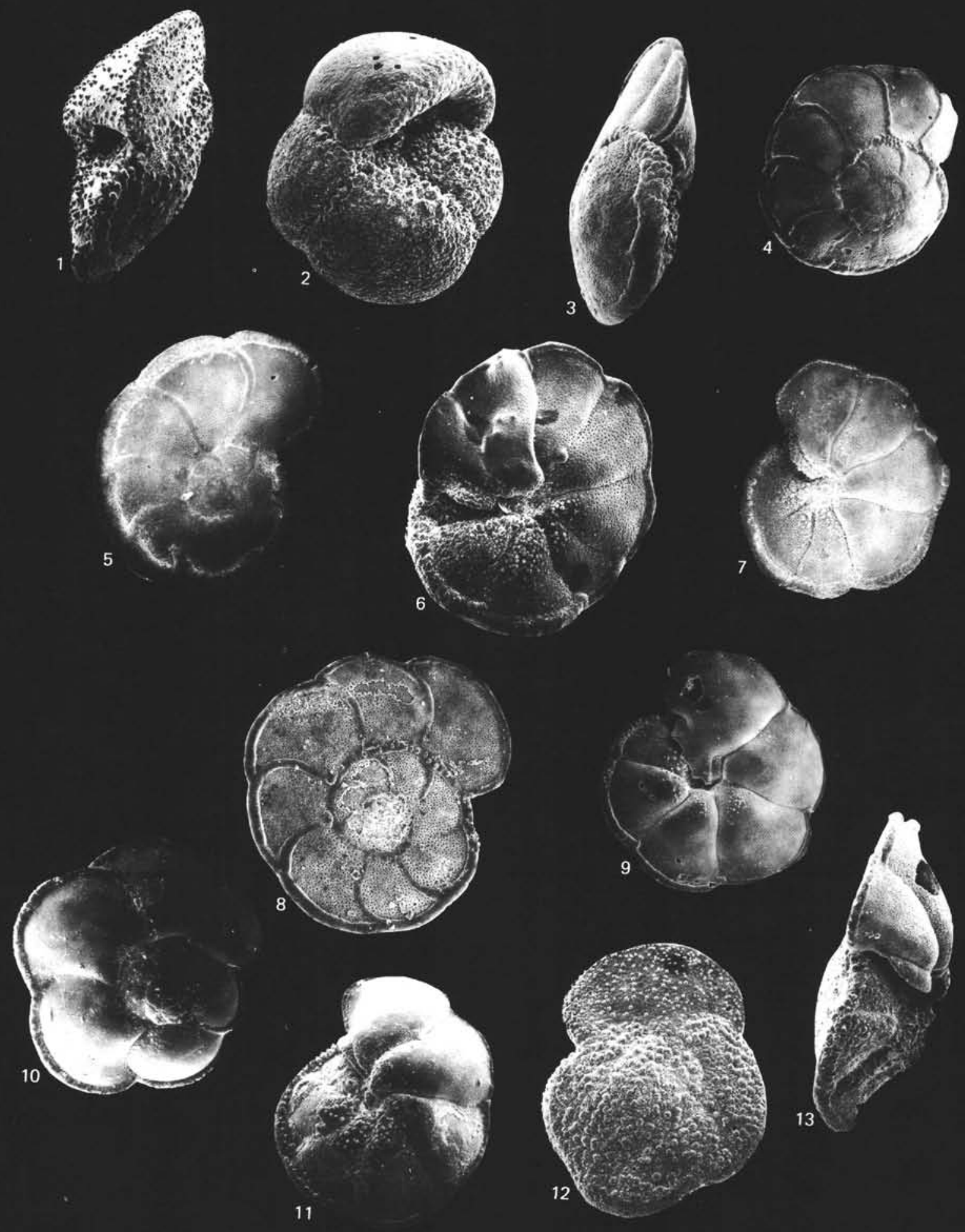

Plate 19. 1, 8, 12. Sample 516-4-1, 30-32 cm, (1) Globorotalia crassula Cushman and Steward, side view, $\times 70$, (8) Globorotalia miocenica Palmer, spiral view, $\times 65,(12)$ Globorotalia crassula Cushman and Steward, spiral view $\times 65$. 2. Globorotalia inflata (d'Orbigny), Sample 516A-6-2, 50-52 cm, umbilical view, $\times 65$. 3. Globorotalia miocenica s.1., Sample 516-5,CC, side view, $\times 65$. 4, 6. Sample 516-4-3, 30-32 $\mathrm{cm}$, Globorotalia pertenuis Beard, (4) spiral view, $\times 35,(6)$ umbilical view, $\times 65$. 5, 7. Sample 516-4-2, 30-32 cm, $\times 45$, (5) Globorotalia multicamerata Cushman and Jarvis, spiral view, (7) Globorotalia miocenica Palmer, umbilical view. 9-11, 13. Sample 516-3,CC, (9) Globorotalia pertenuis Beard, umbilical view, $\times 55,(10)$ Globorotalia exilis Blow, spiral view, $\times 55,(11,13)$ Globorotalia miocenica Palmer (11, umbilical view, $\times 70 ; 13$, side view, $\times 90)$. 